Florida International University

FIU Digital Commons

$11-12-2020$

\title{
Sensor Approach for Brain Pathophysiology of Freezing of Gait in Parkinson's Disease Patients
}

Juan Sebastian Marquez Jaramillo

jmarq056@fiu.edu

Follow this and additional works at: https://digitalcommons.fiu.edu/etd

Part of the Electrical and Computer Engineering Commons

\section{Recommended Citation}

Marquez Jaramillo, Juan Sebastian, "Sensor Approach for Brain Pathophysiology of Freezing of Gait in Parkinson's Disease Patients" (2020). FIU Electronic Theses and Dissertations. 4560.

https://digitalcommons.fiu.edu/etd/4560

This work is brought to you for free and open access by the University Graduate School at FIU Digital Commons. It has been accepted for inclusion in FIU Electronic Theses and Dissertations by an authorized administrator of FIU Digital Commons. For more information, please contact dcc@fiu.edu. 


\title{
FLORIDA INTERNATIONAL UNIVERSITY
}

Miami, Florida

\section{SENSOR APPROACH FOR BRAIN PATHOPHYSIOLOGY OF FREEZING OF GAIT IN PARKINSON'S DISEASE PATIENTS}

\author{
A dissertation submitted in partial fulfillment of \\ the requirements for the degree of \\ DOCTOR OF PHILOSOPHY \\ in \\ ELECTRICAL AND COMPUTER ENGINEERING \\ by \\ Juan Sebastian Marquez Jaramillo
}


To: Dean John L. Volakis

College of Engineering and Computing

This dissertation, written by Juan Sebastian Marquez Jaramillo, and entitled Sensor Approach for Brain Pathophysiology of Freezing of Gait in Parkinson's Disease Patients, having been approved in respect to style and intellectual content, is referred to you for judgment.

We have read this dissertation and recommend that it be approved.

$\begin{array}{r}\hline \text { Elias Alwan } \\ \hline \text { Armando Barreto } \\ \hline \text { Wei-Chiang Lin } \\ \hline \text { Corneliu C Luca }\end{array}$

Ou Bai, Major Professor

Date of Defense: November 12, 2020

The dissertation of Juan Sebastian Marquez Jaramillo is approved.

Dean John L. Volakis College of Engineering and Computing

Andrés G. Gil Vice President for Research and Economic Development and Dean of the University Graduate School

Florida International University, 2020 
(C) Copyright 2020 by Juan Sebastian Marquez Jaramillo All rights reserved. 


\section{DEDICATION}

To my ever-loving and supporting family 


\section{ACKNOWLEDGMENTS}

First, I would like to thank my advisor, Dr. Ou Bai, for his support, accessibility, and drive to innovate. Having him as a researcher model has inspired me to be a lifelong learner and to examine problems as opportunities for novelty.

I also thank the members of my committee, Dr. Alwan, Dr. Barreto, Dr. Lin, and Dr. Luca, for taking the time to evaluate and comment on this dissertation. Special thanks to Dr. Luca for introducing me to the field of Parkinson's Disease research.

A cordial acknowledgment to my lab colleagues at the Human Cyber-Physical Systems lab: Roozbeh, Masudur, Rod, Tushar, Tong, Robin, Connie, and Kaida.

This research was supported by the Graduate assistantship from the Department of Electrical and Computer Engineering at Florida International University and the National Science Foundation (CNS-1552163). 
ABSTRACT OF THE DISSERTATION

SENSOR APPROACH FOR BRAIN PATHOPHYSIOLOGY OF FREEZING OF

GAIT IN PARKINSON'S DISEASE PATIENTS

\author{
by \\ Juan Sebastian Marquez Jaramillo \\ Florida International University, 2020 \\ Miami, Florida \\ Professor Ou Bai, Major Professor
}

Parkinson's Disease (PD) affects over 1\% of the population over 60 years of age and is expected to reach 1 million in the USA by the year 2020, growing by 60 thousand each year. It is well understood that PD is characterized by dopaminergic loss, leading to decreased executive function causing motor symptoms such as tremors, bradykinesia, dyskinesia, and freezing of gait (FoG) as well as non-motor symptoms such as loss of smell, depression, and sleep abnormalities. A PD diagnosis is difficult to make since there is no worldwide approved test and difficult to manage since its manifestations are widely heterogeneous among subjects. Thus, understanding the patient subsets and the neural biomarkers that set them apart will lead to improved personalized care. To explore the physiological alternations caused by PD on neurological pathways and their effect on motor control, it is necessary to detect the neural activity and its dissociation with healthy physiological function. To this effect, this study presents a custom ultra-wearable sensor solution, consisting of electroencephalograph, electromyograph, ground reaction force, and symptom measurement sensors for the exploration of neural biomarkers during active gait paradigms. Additionally, this study employed novel de-noising techniques for dealing with 
the motion artefacts associated with active gait electroencephalography (EEG) recordings and compared time-frequency features between a group of PD with FoG and a group of age-matched controls and found significant differences between several EEG frequency bands during start and end of normal walking (with a $\mathrm{p}<0.05$ ). 


\section{TABLE OF CONTENTS}

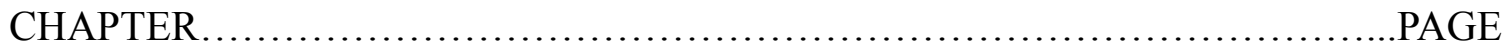

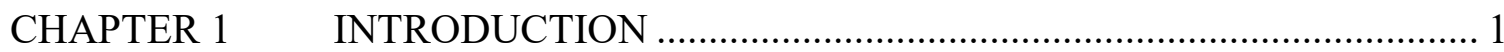

1.1 Parkinson's Disease and Freezing of Gait ..................................................... 1

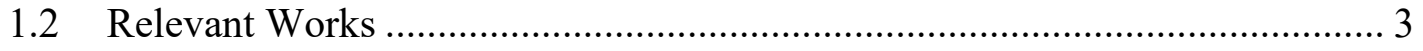

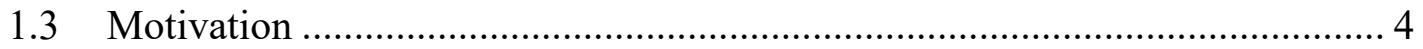

\section{CHAPTER 2 NEURAL CORRELATES OF FREEZING OF GAIT IN PD: AN} ELECTROPHYSIOLOGY REVIEW ................................................................... 7

2.1 Pathophysiology Mechanisms Underlying FoG ............................................. 7

2.2 Literature and Research Methods .............................................................. 12

2.3 Neurophysiological Studies of FoG ……………...................................... 13

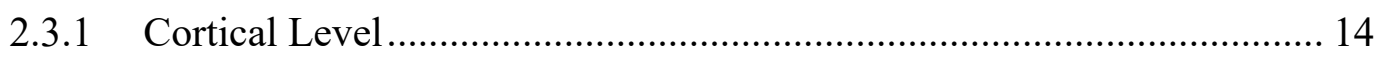

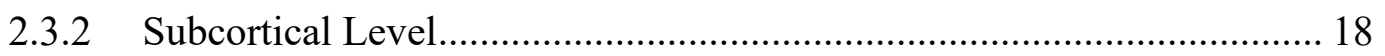

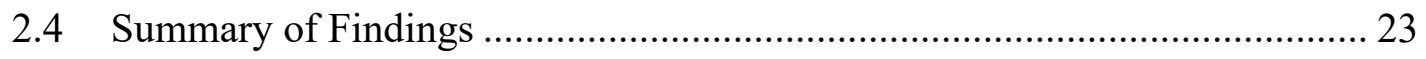

2.4.1 Differences in EEG Between PDFoG + and PDFoG- ........................... 23

2.4.2 Difference STN Activity Between PDFoG + and PDFoG- .................... 23

2.4.3 Difference in the Electrophysiological Activity Between ON and OFF

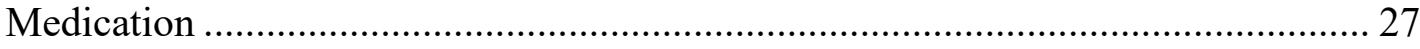

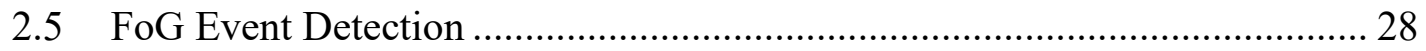

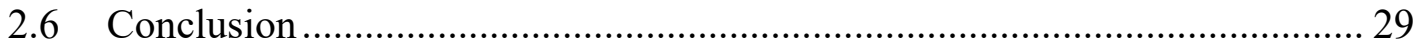

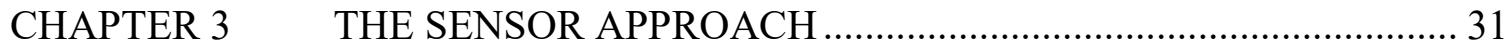

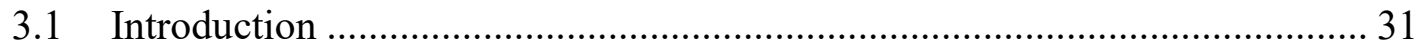

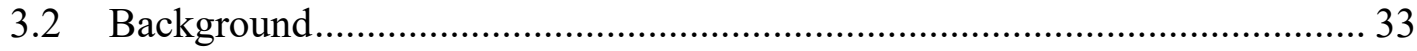

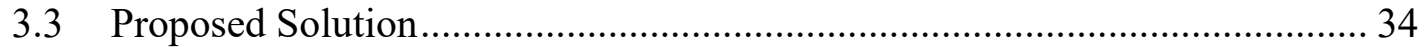

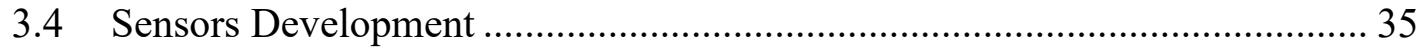

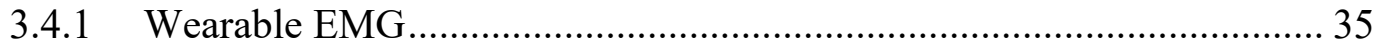

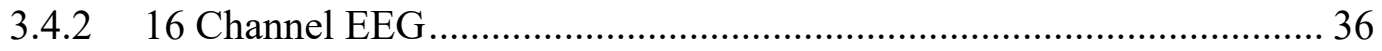

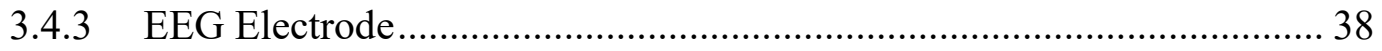

3.4.3.1 Electrode Development ………………………........................... 39

3.4.3.2 Preliminary Dry Electrode Testing................................................... 42

3.4.3.3 Preliminary Dry Electrode Results...................................................... 44

3.4.4 Wearable Ground Reaction Force Sensor .............................................. 47

3.4.5 Wearable Symptom Measurement ......................................................... 48 
3.4.5.1 Preliminary Symptom Measurement Testing ..................................... 48

3.5 Ultra-Wearable Suite Pilot Recording ...................................................... 50

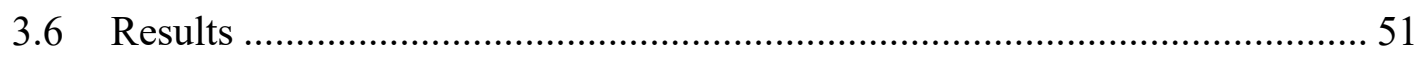

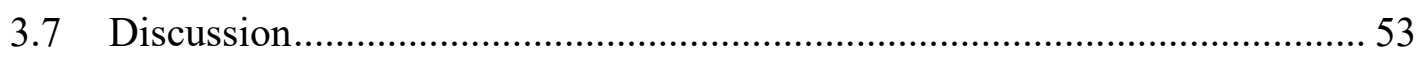

\section{CHAPTER 4 NEURAL BIOMARKERS OF FREEZING OF GAIT IN}

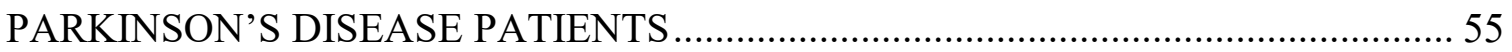

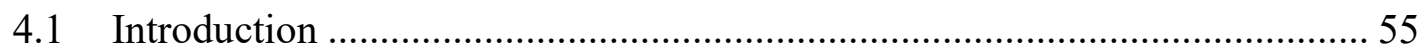

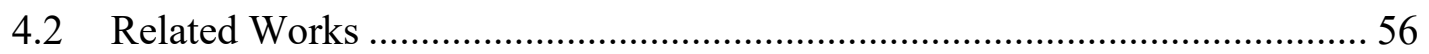

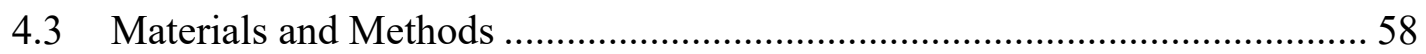

4.3.1 Participants and Experimental Design ................................................... 58

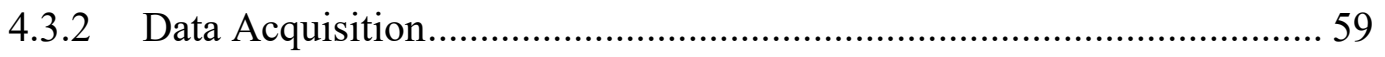

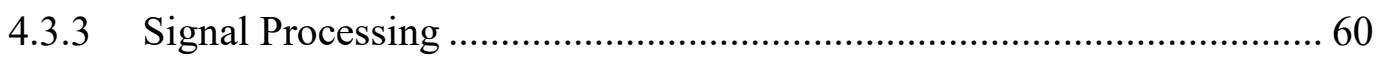

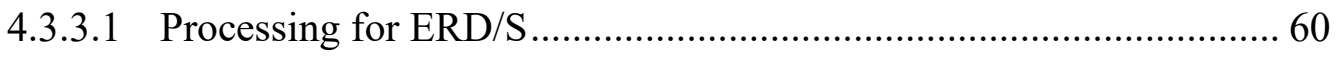

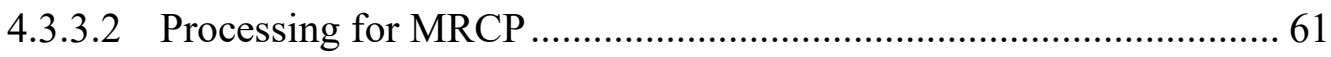

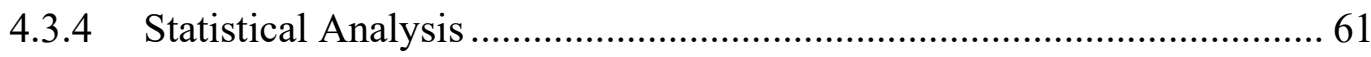

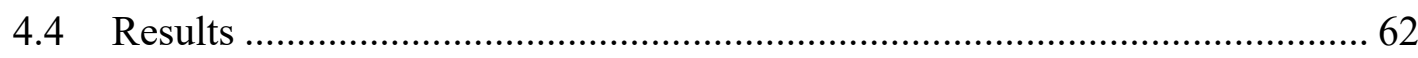

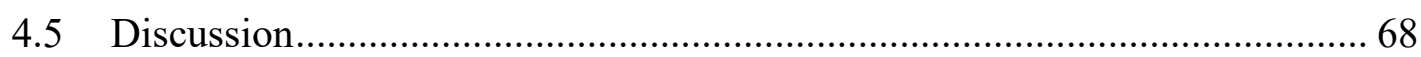

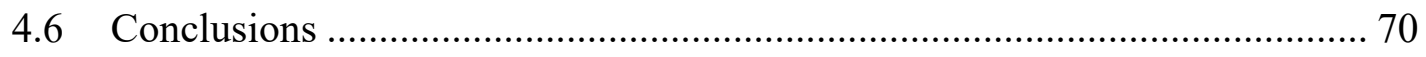

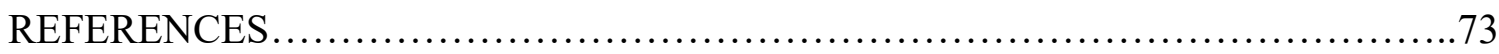

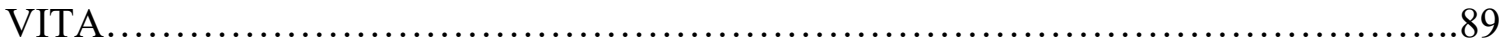




\section{LIST OF TABLES}

TABLE PAGE

Table 1. Results and interpretation of studies that compared neural biomarkers of

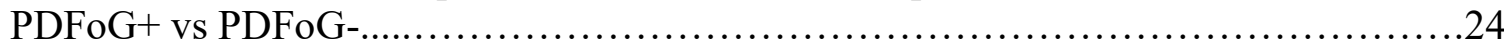

Table 2. Predicted score consensus with SMD rating ............................49

Table 3. Subject demographics (mean \pm standard deviation) .................................59 


\section{LIST OF FIGURES}

FIGURE.

PAGE

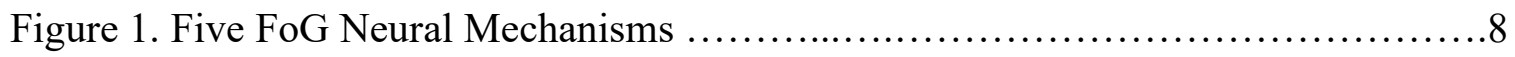

Figure 2. The complete sensor suite, custom-made for active gait neural exploration....34

Figure 3. EMG system with DAQ and electrodes................................. 35

Figure 4. The EEG System............................................... 36

Figure 5. Four lead, individually sprung dry EEG electrode design...................39

Figure 6. Dry EEG electrode range of motion................................... 41

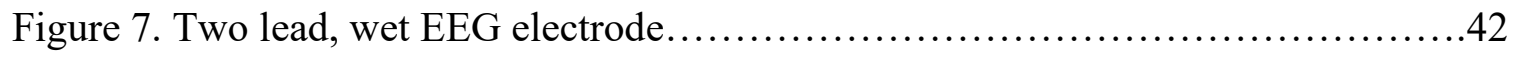

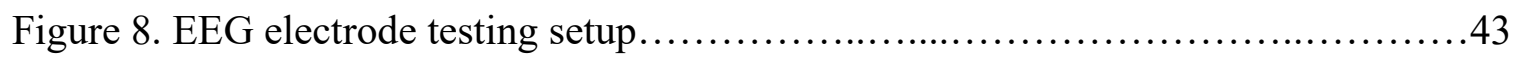

Figure 9. EEG electrode noise and SNR results..................................44

Figure 10. EEG electrode SSVEP results........................................46

Figure 11. Wearable gait analysis system...................................47

Figure 12. Kinematic gait phases from the wearable ground reaction force sensor........47

Figure 13. Wearable movement assessment device with internet connectivity...........48

Figure 14. Motion artefact correction........................................50

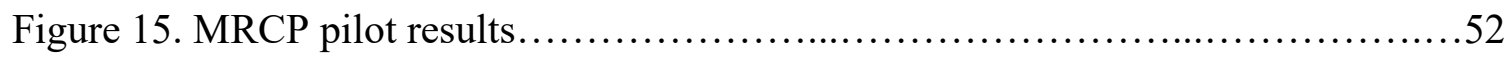

Figure 16. Time-frequency comparison........................................62

Figure 17. At C3, the PDFoG group shows significantly less alpha and low beta desynchronization about $290 \mathrm{~ms}$ after the start of normal walking, compared with HC...63

Figure 18. At CP6, the PDFoG group shows significantly less alpha desynchronization $680 \mathrm{~ms}$ and $290 \mathrm{~ms}$ before the start of normal walking, compared with HC.............64

Figure 19. At $\mathrm{Cz}$, the PDFoG group shows significantly less alpha desynchronization $680 \mathrm{~ms}$ before and $290 \mathrm{~ms}$ after the start of normal walking, compared with HC.........65

Figure 20. ERD differences between HC, FoG-, and FoG $+\ldots \ldots \ldots \ldots \ldots \ldots \ldots \ldots \ldots \ldots 6$ 
Figure 21. Movement-related cortical potentials................................67 


\section{LIST OF ACRONYMS AND ABBREVIATIONS}

ASR

APA

BP

BG

CPG

DAQ

DBS

DCN

EEG

EMG

ERD

ERP

ERS

fMRI

FoG

fpMP

GPe

GPi

GRF

$\mathrm{HC}$

ICA

isMP
Artefact Subspace Reconstruction

Anticipatory Postural Adjustments

Bereitshaftspotential

Basal Ganglia

Central Pattern Generators

Data Acquisition System

Deep Brain Stimulation

Deep Cerebellar Nuclei

Electroencephalography

Electromyography

Event-Related de-Synchronization

Event-Related Potential

Event-Related Synchronization

functional Magnetic Resonance Imaging

Freezing of Gait

frontal peak of motor potential

Globus Pallidus pars externus

Globus Pallidus internus

Ground Reaction Force

Healthy Control

Independence Component Analysis

initial slope of Motor Potential 


\begin{tabular}{|c|c|}
\hline IMU & Inertial Measurement Unit \\
\hline LFP & Local Field Potential \\
\hline LRP & Lateralized Readiness Potential \\
\hline MLR & Mesencephalic Locomotor Region \\
\hline MRCP & Movement Related Cortical Potentials \\
\hline MRI & Magnetic Resonance Imaging \\
\hline PD & Parkinson's Disease \\
\hline PDFoG- & Persons with PD who do no exhibit FoG \\
\hline PDFoG + & Persons with PD who exhibit FoG \\
\hline PMC & Premotor Cortex \\
\hline PMRF & Pontomedullary Reticular Formation \\
\hline PPN & Pedunculopontine Nucleus \\
\hline PwP & Persons with Parkinson's Disease \\
\hline SLN & Supraspinal Locomotor Network \\
\hline SMA & Supplementary Motor Area \\
\hline SMD & Specialist in Movement Disorders \\
\hline $\mathrm{SNc}$ & Substantia Nigra pars compacta \\
\hline $\mathrm{SNr}$ & Substantia Nigra pars reticulata \\
\hline SNR & Signal-to-Noise Ratio \\
\hline SSVEP & Steady-State Visual Evoked Potential \\
\hline STN & Subthalamic Nucleus \\
\hline TA & Tibialis Anterior \\
\hline UPDRS & Unified Parkinson's Disease Rating Scale \\
\hline
\end{tabular}




\section{CHAPTER 1 INTRODUCTION}

\subsection{Parkinson's Disease and Freezing of Gait}

As the second most common neurodegenerative disorder, Parkinson's Disease (PD) affects over $1 \%$ of the population over 60 years of age and is expected to reach 1 million in the USA by the year 2020, growing by 60 thousand each year [1], [2]. It is well understood that PD is characterized by dopaminergic loss [3], leading to decreased executive function causing motor symptoms such as tremors, bradykinesia, dyskinesia, and freezing of gait (FoG) as well as non-motor symptoms such as loss of smell, depression, and sleep abnormalities [4]. Although tremors are one of the most common symptoms, persons with PD (PwP) list falls and gait abnormalities as the most debilitating symptoms, indicating the urgency of focus and leading to the emphasis of this work [5], [6]. FoG affects over half the population with advanced Parkinson's Disease [7]. This highly disabling symptom is defined as "brief episodes of inability to step or by extremely short steps that typically occur on initiating gait or on turning while walking"'[8]. FoG is often responsible for falls within $\mathrm{PD}$ and is highly associated with recurrent falls[9], [10]. Although there are pharmacological, therapeutic, and surgical treatments to alleviate PDFoG, evidence to support their effects is inconclusive[11]. As the leading cause of disabling falls, understanding what causes FoG holds a tremendous public health impact. To date, no accepted model completely explains FoG as this symptom is complex, rare to capture under observation, and varies during manifestation.

A PD diagnosis is difficult to make since there is no worldwide approved test and difficult to manage since its manifestations are widely heterogeneous among persons with Parkinson's Disease (PwP) [12]-[14]. PD patients often develop medication tolerance and 
for some, the medication is not at all effective. In the case of unmanageable symptoms, specialists in movement disorders may choose to perform neurosurgery, which involves the insertion of electrical stimulators deep in the brain that may cost as much as $\$ 100 \mathrm{~K}[15]$. This procedure, however, does not guarantee symptom improvement, as even the location for stimulation depends on what symptom is targeted[16], [17]. Better care strategies that consider the differences between patients are needed. Understanding the PD patient subsets and the neural biomarkers that sets them apart will lead to successful personalized care.

To explore the physiological alternations caused by PD on neurological pathways and their effect on motor control, it is necessary to detect the neural activity and its dissociation with healthy physiological function. Additionally, the branching systems which are affected by the pathology must also be studied. To this effect, great effort has been placed in the understanding of movement-related cortical potentials (MRCPs), a type of neural activity that occurs before voluntary movement, mostly during upper limb tasks. The restrains on MRCPs associated with upper extremities have been partly shaped by the instrument limitations, requiring the avoidance of movement artefacts and the complications brought by multisensory recordings. Furthermore, the challenge of movement artefacts from the recording setups as well as the noise that comes from active gait, affect experiment feasibility and reliability, deter previous efforts to understand the connections between neural conditions and active gait. Hence, there is still a knowledge gap for the relationship between cortical modulations and active gait showing altered pathophysiology in PD patients who exhibit FoG. 


\subsection{Relevant Works}

FoG can be provoked by different triggers including turning, multi-tasking, and emotional states [18]. Due to the nature of FoG and its multi-systemic neural impairments, disturbances to individual brain regions are difficult to isolate. This further complicates the understanding of this symptom, which sometimes causes medication intervention and treatment to be unfocused, non-specific, and unsuccessful. To this effect, many research groups have used a variety of methods and approaches to understand the pathophysiological mechanisms behind FoG. Among the most notable approaches are behavioral and clinical measures ranging from gait and posture[6], [19]-[24], upper limb biomechanics[25], jaw displacement[26], saccade latency, velocity, and gain[27], audiospinal reflex[28], handwriting measures[29], foot pedal measures[30], speech[31], electromyography (EMG) [32], [33], and even rapid eye movement frequency[34]. However, to explore the physiological changes specifically related to FoG in neurological pathways and their effect on motor control, it is necessary to detect efficacious neural biomarkers that may differentiate PD from healthy controls and PD subtypes from one another. To accomplish this, the research tools that have been applied include functional near-infrared spectroscopy [35], EEG [24], [36]-[39], deep brain electrode-based recordings[37], [40]-[44], and various neuroimaging tools such as functional magnetic resonance imaging (MRI) volumetric MRI, or diffusion MRI [45]-[48], and positron emission tomography [46], [47]. It is well known that each of these methods has a benefit over the other, for example, the spatial vs temporal resolution advantage held by fMRI over EEG, or the non-invasive vs surgical advantage of EEG over deep brain electrode-based recordings. 
Recent studies that focus on clarifying the mechanisms behind FoG rely on functional MRI, which are restricted to motor imaging [45], [49], or EEG and are limited to minimal executive motor control, such as finger flexion [50]. To the author's knowledge, only one other study has attempted to examine the mechanisms underlying FoG for PwP using EEG during active gait [51]. This study found that a reduction in the early slope of the MRCP was consistent with stride length reduction, and based on their findings, they further state that $\mathrm{PwP}$ will exhibit electrical differences both in the preparatory and executive portions of gait [52]. However, the group also goes on to discuss its experimental flaws, including the short gait cycle duration, composed of only 3 steps, which in turn led to the inability to guarantee voluntary movement and automaticity after initiation. Similar studies, focusing on $\mathrm{PwP}$ and FoG, have focused on evaluating the power spectral densities calculated from EEG [53], [54], or corticomuscular coherence in non-FoG PwP [55]. This work will aim to combine whole-body sensing, composed of wearable EEG, ground reaction force (GRF), EMG, and behavioral outcomes for FoG pathophysiology exploration.

\subsection{Motivation}

The proposed study has only recently been made possible, thanks to advances in interconnectivity allowing for synchronous recordings of the modalities mentioned above. The miniaturization of integrated circuits, the multi-sensory fusion to remove motion artefacts, and both the cost and battery efficiency have permitted for research to be taken outside of the lab and into more real settings, allowing for longer recordings, more natural experimental paradigms, and more applicable conclusions[56]-[61]. Although the systems have been proven in other applications, we expect to face difficulties in data collection, processing, and analysis. An active gait neural recording often consists of multiple 
repetitions of the same task, usually ranging from forty to upwards of three hundred trials of repeated events. As the proposed study will focus on PD, a cognitive and motor impairing disease, new hardware and software platform for accurate measurement of cortical activity associated with active gait is proposed to overcome signal corrupting motion artefacts. Once data is rid of motion artefacts and been successfully processed, data analysis will institute the continuation of the work, as interpretation will rely heavily on a thorough understanding of the neurophysiology and proposed underlying mechanisms behind FoG biomarkers. Success will thus be contingent on finding biomarkers that differentiate PwP with FoG and healthy control. The proposed work will be divided into the following:

Task (1): Develop and test an ultra-wearable, whole-body suite capable of sensing neural features associated with supplementary motor area activity during active gait.

Task (2): Ensure related cortical potentials recorded using the sensor suite are rid of stationary and non-stationary artefacts.

Task (3): Clinical feasibility of the ultra-wearable neural exploration suite during active gait paradigms

Question 1: Whether the amplitudes of Bereitshaftspotentials (BP), an early component of MRCP, or other neural features are affected in PD patients while performing preparatory active gait compared with healthy controls.

Questions 2: Whether MRCP is altered following Levodopa administration in PD patients with and without FoG during active gait compared with healthy controls. 
The intellectual merits of this work will rise from the implementation of smart sensing technologies in the application of neurophysiology exploration. This work will explore novel synchronous wearable recordings of non-invasive top-down sensors for whole-body event analysis. The analysis and multisensory implementation, consisting of electrical potentials from scalp electrodes in electroencephalography, skin-surface electromyography, lower and upper limb features related to symptoms and locomotion will (1) yield motion artefact attenuation from multisensory synchronization of wearable sensors capable of delivering reliable and valid data; (2) allow for a better understanding of FoG pathophysiology and its effects on neural pathways and lower limbs during active gait, and (3) expand pathophysiology research bounds by the validation of multisensory active gait paradigms. 


\section{CHAPTER 2 NEURAL CORRELATES OF FREEZING OF GAIT IN PD: AN ELECTROPHYSIOLOGY REVIEW}

\subsection{Pathophysiology Mechanisms Underlying FoG}

Locomotion is a complex process that involves both automatic, emotional, and volitional control [62]-[64]. In non-automatic gait, the initiation command is generated at the cerebral cortex and is executed by the thalamocortical, corticobulbar, and spinal projections networks [62]. Automatic rhythmicity, posture preparations, and adjustments during locomotion are regulated by the brainstem and spinal cord after gait is volitionally initiated. Meanwhile, the cerebellum simultaneously takes in the sensory feedback from the spinal cord and feed-forward information from the cortex to regulate predictive control [65]. In PD, multilevel network failure may ultimately lead to FoG events. However, the neural complexity leads to uncertainty in identifying and isolating specific neural impairments that result in FoG. To explain the pathophysiology, five non-exclusive mechanisms have been suggested regarding the physiological alterations leading to FoG [8], [18]. 


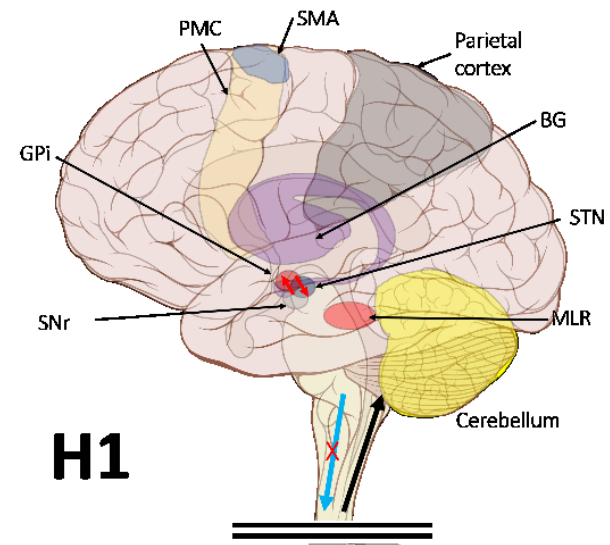

- CPG (For locomotion)

es.

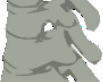
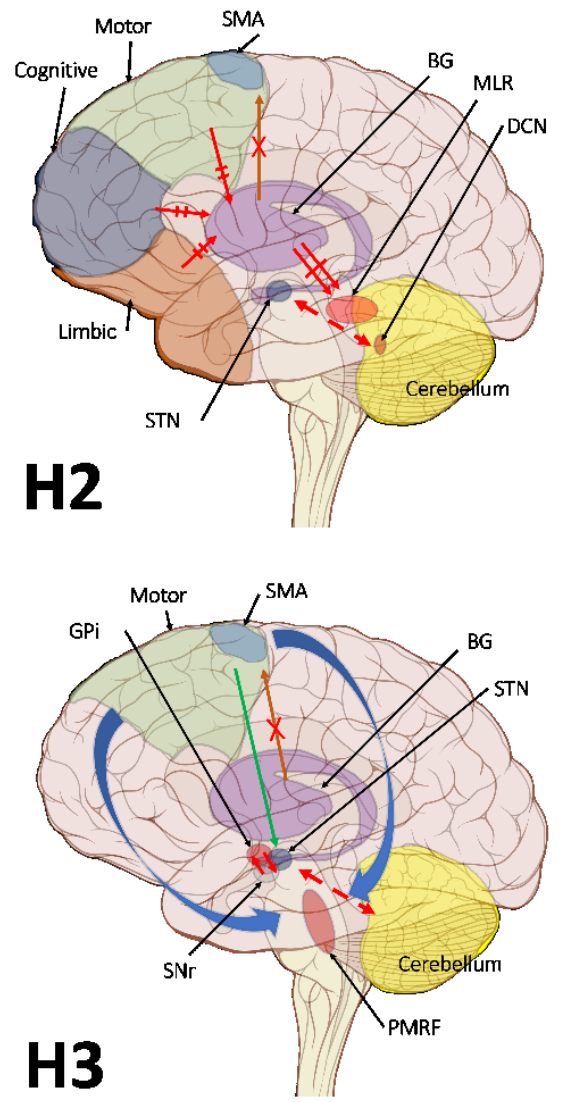
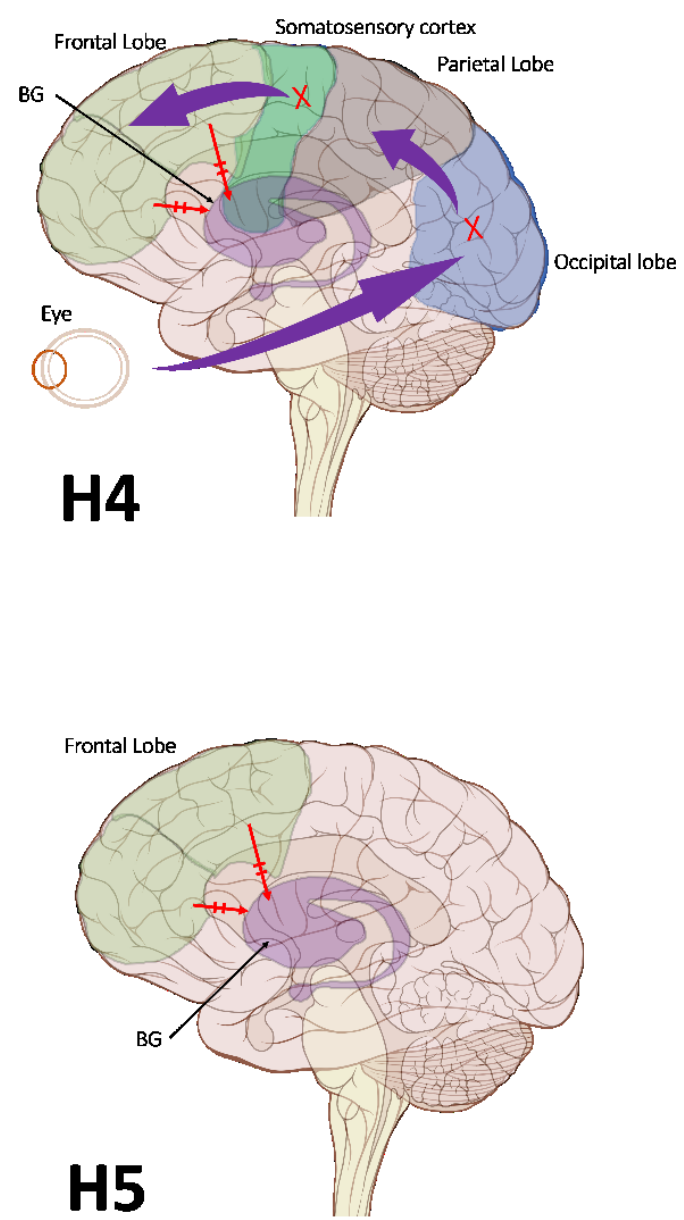

Figure 1. Five FoG Neural Mechanisms. H1. Abnormal control outputs from central pattern generators. The supraspinal locomotor network (SLN) is composed of the premotor cortex (PMC), supplementary motor area (SMA), parietal cortex, basal ganglia (BG), subthalamic nucleus (STN), mesencephalic locomotor region (MLR), and cerebellum. $\downarrow$ In normal function, the SLN network sends cues for turning, stopping, obstacle maneuvering, and new locomotion goals. X In PD, FoG is caused by the disruption of SLN cues. $\downarrow$ Disruption is caused by inhibition of the globus pallidus internus (GPi)/ substantia nigra pars reticulata $(\mathrm{SNr})$ pathway resulting in decreased rhythmic control. H2. A disconnect 
between the basal ganglia and the supplementary motor area or cross talk to basal ganglia. $\uparrow$ In normal function, BG-SMA sends internal cueing for automatic initiation of previously learned tasks. X In PD, FoG is caused by the disruption of BG-SMA cues. This disconnect leads to the inability to multitasking. $\hat{i}$ The increased inhibitory output from deep cerebellar nuclei (DCN) further prevents the execution of habitual responses. $=$ In PD, crosstalk between the input to BG from the cognitive, motor, and limbic cortices may also trigger FoG. $\nRightarrow$ Firing in the output nuclei of the BG inhibits MLR leading to FoG events. H3. Knee-trembling and the abnormal coupling between posture and gait. $\uparrow$ In normal function, BG-SMA sends internal cueing for the automatic initiation of previously learned tasks. Because of the dopamine depletion, executive function is lost. X The BG then fails to convey appropriate anticipatory postural adjustments. $\downarrow$ The breakdown of coupling between posture preparation (at the SMA) and step initiation (in the Motor cortex) might occur at the pontomedullary reticular formation (PMRF), which plays a role in postural control and regular locomotion. $\downarrow$ The hyperdirect pathway (SMA-STN) becomes engaged as a result of the coupling breakdown activating the GPi/SNr pathway. $\hat{i}$ Additionally affecting cerebellar automatic gait processing. $\downarrow \mathrm{GPi} / \mathrm{SNr}$ oscillations may underpin characteristic $3-8 \mathrm{~Hz}$ knee-trembling. H4. A perceptual malfunction and slowing down when passing doorways. $\uparrow$ In normal function, the dorsal stream takes visual information to the occipitoparietal stream, where somatosensory signals are then transferred to the frontal lobe for the origination of motor function intent. X In PD, FoG events are caused by dysfunctional dorsal stream processing, which consequently causes inappropriate adaptation of locomotion. H5. A Consequence of frontal executive dysfunction. $\neq$ In PD, FoG events are caused by a disconnect between the frontal lobe and the BG. This disconnect results in poor performance of multiple consequent tasks and the characteristically low frontal assessment battery and verbal fluency scores.

The first hypothesis states that the characteristic gait features associated with FoG are caused by abnormal control outputs from the central pattern generators (CPG) in the spinal cord [6], Figure $1 \mathrm{H} 1$. The CPG is a neural network in charge of goal-directed motor output independent of external timing cues, or sensory feedback [62]. In addition to rhythmic control, the CPG also receives commands from the supraspinal locomotor network, which is made up of the primary motor cortex, the supplementary motor cortex, the parietal cortex, the BG, the subthalamic nucleus, the mesencephalic locomotor region, and the cerebellum[63]. In PD, FoG events may be caused by a disruption of the supraspinal control cues to the CPG, which are crucial for turning, stopping, maneuvering obstacles, and adapting to new locomotion goals [8]. This explanation takes into consideration the fact that in the control pathway of healthy individuals, the sensorimotor striatum that is in charge of habitual behavior [64], is inhibited by the substantia nigra pars compacta (SNc) through D2 dopamine receptors, which are involved in locomotion, learning, memory, and 
reproductive behavior [65]. During normal operation, inhibition of the striatum results in inhibition of the globus pallidus pars externus (GPe). The active GPe then allows for inhibition of the globus pallidus internus/substantia nigra pars reticulata pathway (GPi/SNr) resulting in the execution of previously learned tasks [66]. In the case of PD, the lack of synergy between dopaminergic modulation networks leads to inhibitions of the GPi/SNr pathway resulting in decreased rhythmic gait control [8], [66]-[69]. Although not specifically directed to the focus of this review, it is worth noting that the formerly popular direct/indirect pathway's simplistic assumptions regarding loops and purely excitatory/inhibitory connections have been questioned and debunked to a significant extent [70].

The second mechanistic hypothesis underlying the pathophysiology of FoG states that due to the disconnect from the BG to the SMA, the internal cueing that automatically initiates previously learned movements is interrupted [71], Figure $1 \mathrm{H} 2$. Some studies considered that FoG might be due to the loss of automatic updating of motor programs by the dysfunctional BG in PD [54], [67], leading to the inability to multitask[8], [68]. Furthermore, because of the increased competition between the excitatory output from the STN, increased inhibitory output on the deep cerebellar nuclei hinders the ability to rely on previously learned habitual responses [8], [69]. Moreover, FoG might be triggered by crosstalk of complementary, yet competing, BG inputs from motor, cognitive, and limbic cortical areas [8]. In this model, the synchronous firing in the output nuclei of the BG leads to increased inhibition in brainstem locomotor areas and consequently to FoG events [69]. The associative striatum and frontal-parietal cortices are relatively spared early in the disease course, allowing PwP who exhibit FoG (PDFoG+) to operate gait through goal- 
directed strategies. However, as a result, their gait becomes less automated and vulnerable to interference from concurrent task demands that can disrupt gait control [68].

From the observation of the characteristic knee-trembling during FoG, the third hypothesis states an abnormal coupling between posture and gait, which leads to multiple anticipatory postural adjustments (APA) preceding FoG events [8], [72], [73], Figure 1 H3. This finding suggests that the inability to couple normal APAs with the stepping motor pattern is what causes FoG events in PD during a forward imbalance. APA needed for normal gait initiation, resulting in a frozen state of attempted readiness [74]. Breakdown in the coupling between posture preparation by the SMA and step initiation by the motor cortex might occur in the pontomedullary reticular formation [8], [72]. The hyperdirect pathway likely becomes engaged as a result of increased response conflict, activating the $\mathrm{GPi} / \mathrm{SNr}$ while also disrupting cerebellar processing involved with automated gait modulation. Altered 3-8 Hz oscillations between the STN and GPi may also underpin the characteristic 'trembling in place' often observed during FoG [18], [75].

The fourth hypothesis states that the FoG event is caused by a perceptual malfunction, which in association with online planning, causes $\mathrm{PDFoG}+$ to decrease speed as a response to the visual input related to locomotion planning [76], [77], Figure $1 \mathrm{H} 4$. Almeida and Lebold found decreased step length and increased gait variability while approaching a narrow doorway, for PDFoG $+[78]$, [79]. However, the previous study showed that PDFoG + do not overestimate door widths more than PwP who do not exhibit FoG (PDFoG-), instead, they misjudge the speed reduction resulting from passing through doorways [80]. The inappropriate activation of locomotor adaptation is considered as the 
result of dysfunctional dorsal stream processing [81], which helps with spatial location and guides movement response [82].

The fifth hypothesis states that FoG is a consequence of frontal executive dysfunction, where there is a disconnect between the BG and the frontal lobe, resulting in poor performance when trying to achieve multiple tasks, Figure $1 \mathrm{H} 5$. This is further supported by low scores in frontal assessment battery scores and verbal fluency in PDFoG + compared to PDFoG- [18], [75], [83]. Extra-nigral pathology impairs compensatory attentional gait strategies and contributes to levodopa resistant FoG, especially with PD progression [68].

The various hypotheses are a testament to the complexity of the underlying mechanisms of FoG, likely the heterogeneity of contributing factors among patients. Therefore, continued exploration of the pathophysiology of FoG in PD is needed. Moreover, not all PD who exhibit FoG events are triggered by the same causes or respond equally to medications or therapy. Those who may only experience events when not under observations, make FoG evaluation and understanding in the clinic elusive [18]. This review concentrates on dissecting the previous works that relied on electrophysiology to analyze neural biomarkers associated with FoG. For neuroimaging studies delving into PDFoG, the reader is encouraged to refer to the recent review papers [47], [48].

\subsection{Literature and Research Methods}

Following the Preferred Reporting Items for Systematic Reviews and Meta-Analyses statement [84], the eligibility criteria for articles included those published before March 2020, written in English, and currently under published status as journals or full-length conference articles. PubMed was used as the primary information source with the following search query “EEG OR LFP AND Parkinson's Disease AND Freezing of Gait”. The search 
query was conducted by two (2) independent authors who then combined the resultant findings. This search yielded a result of 34 articles from which 10 articles were removed. Six were removed as the terms "Freezing of Gait", "EEG", or "Parkinson's Disease" were used during the introduction part but not as part of the research objective [25], [85]-[89], the other four were removed because the articles did not focus on electrophysiology for its methods [35], [90], because the article was either a poster abstract and not yet a full publication [91], or because the article did not deal with human subjects [92]. To complement the neurophysiology review aspect of this paper, in addition to the articles found through the PubMed search, sources such as Scopus or Google Scholar were used when clarifications were needed.

\subsection{Neurophysiological Studies of FoG}

Recent advances in wearable electronics and computing have allowed for the miniaturization of electronics and the transition from bulky instrumentation to wearable and non-intrusive tools. This shift has allowed for neural biomarker explorations in environments that closely match everyday activities [93] and even allowed for the implementation of protocols during states of high activity, such as walking [94], [95] and cycling [96], [97]. Such daily activity paradigms allow for a more precise interpretation of results because parallel functions in studies using motor imagery [98] or treadmill walking[86] have to be considered. Furthermore, the constraints of simplified tasks of finger/wrist flexion to avoid motion artefact [99], [100] are no longer a big concern [68], [101], [102]. Furthermore, although some of the studies resulting from the PubMed query did not rely on wearable systems for their instrumentation, their findings can be translated into current and future studies using such wearable technology. This review begins with 
the cortical studies that use EEG to explore neural biomarkers and/or behavioral measures, then moves deeper into subcortical studies while discussing the relevant cortical and subcortical loop connections

\subsubsection{Cortical Level}

Locomotion is a complex process involving gait initiation at the cerebral cortex and automatic rhythmic execution regulated by the brainstem and spinal cord. Another important contributor is the simultaneous predictive control through feed-forward processing by the cerebellum. These intricate mechanisms may account for the lack of consensus in FoG pathophysiology, given that exploring the complete projection system results in an overwhelming analytical task. The difficulty to selectively study individual networks or components arises because these networks are all inter-relatedly engaged during real-life locomotion. In the case of EEG, pyramidal cells account for most of the scalp-recorded electrical signals. This non-invasive tool is thus frequently used in the exploration of the cerebral cortex, which is shown as the processing center for proprioception, predictive operations [103], and APA [104], [105]. Of the 24 resulting studies from the search query, 16 studies used EEG in their approach to detect or predict FoG events or study neural changes with FoG.

For FoG biomarker exploration, several studies have focused on the study of the cortical activity associated with self-paced upper limb motion in EEG [50], [99], [106]-[108]. A negative potential, which starts 1-2 seconds before the limb movement, has been found in EEG electrodes over the central area, namely the readiness potential or BP. The BP is a part of the slow cortical movement-related potentials and is followed by the pre-motor positivity [109]. Early BP, which occurs 1-2 s before limb movement, is associated with 
activation from the pre-supplementary motor area (pre-SMA), SMA, and Brodmann area 6 [109]. The late BP that occurs between 400-500 milliseconds before limb movement is associated with activation of the primary motor cortex [110]. Studies have demonstrated that the amplitude of early BP is reduced in persons with PD compared to healthy controls [108], [111], [112]. In contrast to the amplitude reduction in the early BP, late BP amplitude is increased in PD compared with healthy persons. The increased BP amplitude may indicate a compensation mechanism used to achieve limb control in the absence of information handover from the BG to the SMA [108]. These results have been largely reproduced only in persons with PD while performing upper limb tasks [109], due largely to motion artefacts abundant with movements of other parts of the body. In addition to BP, Butler et al. investigated the lateralized readiness potential (LRP) associated with cued response to the target detection tasks [39]. They found a significantly earlier onset and a larger amplitude of the LRP in PDFoG+ compared to PDFoG-. This indicates excessive recruitment of lateral premotor areas due to the loss of automatic motor control. Their finding is in agreement with the second mechanism hypothesis, suggesting that deficit in attentional set-shifting is caused by loss of automatic updating of motor programs due to the dysfunctional BG-SMA pathway [8], [39], [68].

Previous studies related to lower limb control have mainly investigated the difference between participants with PD and healthy controls [113]. Relations between stride length changes and MRCP have also been explored in PDFoG+ and PDFoG- [114]. The study by Shoushtarian et al. found that a reduction in the early slope of the MRCP was coupled with stride length reduction for PDFoG- but not for PDFoG+. Their findings show electrical evidence that cortical disturbances correlate with stride length reduction in PDFoG-, 
whereas the PDFoG+ showed no stride length relationship [114]. However, this study holds several experimental design limitations including the short gait cycle duration, consisting of only 3 steps, which is not enough to guarantee gait programming is generated by automation instead of attentional strategies. Similar studies on neural signatures of FoG have focused on evaluating the cortico-muscular relationship between the healthy elder and PDFoG+/PDFoG- [55], [115]. Among them, Gunther et al. found a pronounced relationship between EMG and EEG at the beginning of FoG events and voluntary stops. However, this finding was not discussed in the context of its physiological explanation, and there was no significant difference between the voluntary stops and FoG events [115].

In addition to the MRCPs, time-frequency analysis is another method for evaluating neural patterns. This approach involves the evaluation of frequency bands [116], [117], event-related synchronization (ERS), and desynchronizations (ERD) [118], as well as information flow to examine how a physical manifestation can be correlated to a neural occurrence. In the work by Singh et al., theta, and beta bands were evaluated during lower limb pedaling in PDFoG+ and PDFoG- [119]. Results from this study showed that compared to PDFoG-, PDFoG+ exhibited an attenuated mid-frontal theta $(4-8 \mathrm{~Hz})$ power, which is associated with impairments in cognitive control [120], [121]. An increased beta (13-30 Hz) power was also found in PDFoG+ compared to PDFoG-. Because beta-band oscillations are considered a result of interactions between the frontal region and the BG [37], [122], [123], an increased beta suggests an increased coupling between the frontal region and the $\mathrm{BG}$ in $\mathrm{PDFoG}+$ as compared to PDFoG-. Moreover, in the study of electrophysiological signature during actual FoG events, Shine et al. found an increase in theta band power during FoG episodes within the central and frontal areas [117]. This result 
suggests that FoG is related to the frontoparietal processing of conflict-related signals [46], [124], [125]. In a later study, the same group found an increase in beta activity over the parietal area during the transition from normal walking to FoG events. The increased beta activity indicated that the frontal generated motor plans failed to reach the motor cortex, resulting in the FoG events [126]. Similar STN coherence findings suggest a relation to parkinsonian limb tremor frequencies $(2-10 \mathrm{~Hz})$, in deep brain recording studies [127]. Further works by Toledo et al. during seated position also found higher high-beta (21-35 $\mathrm{Hz}$ ) activity during the OFF dopaminergic medication, in the STN for PDFoG+, versus PDFoG- [37]. During the ON dopaminergic state, the high-beta power in PDFoG+ was reduced in addition to FoG event cessation. Furthermore, coherence was found between the low-beta component (at $\mathrm{C} 3$ and $\mathrm{C} 4$ ), as well as, the high-beta coherence (between the STN and the SMA at Cz). Altogether, this study indicated FoG events are caused by a malfunction of the frontal cortex-BG networks via the motor and associative STN loops [37].

In addition to the studies of neural correlates between EEG oscillations and lower limb motor execution, Tard et al. explored motor preparation preceding motor execution, which is altered in PDFoG+ [128]. In this study, an oddball discrimination task was used to compare the event-related desynchronization (ERD) and ERS of PDFoG+, PDFoG-,, and age-matched healthy controls. Results showed that even though all groups discriminated the target from the random stimuli, as shown by the event-related potential (ERP) following the auditory preparatory stimuli, no significant beta ERD was observed in PDFoG+ compared to the other two groups. This indicated that although the PDFoG+ correctly perceived the stimulus, their post-perceptual and integrative processing might have been 
impaired [37], suggesting a physiological dysfunction between the frontal cortex and the BG within the motor and associative STN loops. The BG alterations were related to either hypersynchronized thalamocortical activity or reduced BG involvement [129], [130]. Additionally, the longer ERS in PDFoG+ is a marker of the high power beta oscillation in the STN, which results from the corticosubthalamic synchronization at the midline area [131]. In a visual feedback study, Velu et al. studied the cortical information flow between the occipital $(\mathrm{Oz})$, parietal $(\mathrm{P} 4)$, and motor $(\mathrm{Cz})$ networks between six healthy controls and two PDFoG+ (one who was responsive to the visual feedback and one who was not). This study found a decrease in the beta power band in the response PwP when the visual cues were presented. Furthermore, the responsive $\mathrm{PwP}$ showed an increased beta band information flow from the visual area to the motor area, from the visual area to the parietal area, as well as, a decrease in the power of delta and alpha bands. The beta band power decrease and information flow findings suggest a correlation between the visual cue and movement initiation [132].

\subsubsection{Subcortical Level}

Utilizing EEG for gait exploration is a noninvasive way of analyzing the initial phases of locomotion, which are generated at the cerebral cortex. However, after the gait is initiated, thalamocortical, corticobrainstem, and spinal projection produce motor programs that result in rhythmic gait control [105]. Additionally, both the pedunculopontine nucleus (PPN) [44], [105], [133] and STN [37], [40], [42], which are located deep in the brainstem, have been considered to be largely involved in the gait failure for persons with PD. Among the 24 studies from the search query, eight studies used subcortical recordings to either: study the deep brain stimulation (DBS) frequencies that resulted in the most FoG reduction 
[134]; or evaluate frequency band changes during the seated state [37], stepping as a walking surrogate state [42], [44], bicycling state [43], [97], or during an active walking state [40], [41].

Anidi et al. investigated whether beta oscillations can be used to differentiate PD FoG phenotypes under no DBS, DBS at $60 \mathrm{~Hz}$, and DBS at $140 \mathrm{~Hz}$ frequencies from eight PDFoG+ and four PDFoG-. They observed that the beta burst durations were significantly longer in PDFoG+ compared to PDFoG- when walking in place or forward walking. Additionally, for PDFoG+ during stepping while freezing beta bursts were longer than during stepping without freezing. Both 60 and $140 \mathrm{~Hz}$ DBS stimulation resulted in improved gait arrhythmicity in freezers compared to no stimulation. During stepping in place, $60 \mathrm{~Hz}$ and $120 \mathrm{~Hz}$ stimulation attenuated beta burst power. Though $60 \mathrm{~Hz}$ stimulation had a greater effect in reducing the beta burst power, both stimulation frequencies attenuated the beta burst power during forward walking [134].

Toledo et al. reported a relationship between STN activity and EEG frequency coherence while subjects were sitting. During the OFF state with deprivation of levodopa administration, $\mathrm{PDFoG}+$ showed higher high-beta $(21-35 \mathrm{~Hz})$ activity in the STN compared to PDFoG- [37]. During the ON state with levodopa administration, similar highbeta levels were found in both the PDFoG+ and PDFoG- groups. They considered that the high-beta power reduction may have been mediated by levodopa administration which also suppressed the FoG occurrence. Altogether, this study provided a solid basis to support the pathophysiological mechanism leading to FoG, indicating that FoG events are caused by a malfunction of the frontal cortex-BG networks via the motor and associative STN loops [37]. 
In experiments where movement is restricted due to the recording setup or the movement artefacts contamination [42], stepping in place is used as a surrogate for actual gait, because stepping involves both balance control and rhythmic activity similar to the actual gait. Moreover, stepping in place is even susceptible to FoG events [22], [135]. The following two studies implemented stepping in place paradigms as surrogates for active gait, one focusing on the changes to the PPN [44] and the other on changes to the STN [42]. Fraix et al. explored the oscillatory changes of the PPN in seven PDFoG+ with DBS. Their study included DBS recordings of bilateral PPN and cortical needle EEG at central and frontal areas. Their main finding was increased alpha $(5-12 \mathrm{~Hz})$ oscillations during stepping in place while ON levodopa compared with OFF levodopa. Contrary to other studies, they reported a beta power decrease while ON levodopa compared with OFF levodopa. This discrepancy was attributed to differences in the experimental setup as well as the long duration of STN DBS stimulation, which could have led to plastic changes in the PPN. This study also reported decreased gamma activity in the PPN while ON levodopa. This decreased gamma-band activity was associated with increased PPN-Cortical coherence in the gamma band. These findings supported increased phase locking between the cortex and the PPN in the gamma band, which is in agreement with an MRI study showing a direct pathway between the PPN and the frontal cortex [44], [136]. Fischer et al. investigated neural dynamics in the STN in persons with PD while subjects were seated and stepping in place. Their main findings include a suppression in the beta band $(20-30 \mathrm{~Hz})$ after ipsilateral heel strikes, which was distinct between the left and right STN sites when alternating stepping cycles. Furthermore, this study found that the auditory cueing resulted in an increased beta modulation and more synchronized gait regulation. The increased beta 
modulation leads to the consideration that alternate left-right DBS may redistribute beta bursts similarly to auditory cueing [42].

Bicycling is a novel experimental paradigm used to elicit movement-related neural responses with less movement artefact contaminated compared to actual gait. Research has shown that PDFoG + may bicycle regardless of their severe walking alterations [96]. Recent findings also show that cycling even promotes acute and sustained symptom improvement [137]. Bicycling was associated with a stronger sustained cortical activation as indicated by a high beta power decrease. Meanwhile, cortical motor demand was decreased during sustained cycling [43]. In the works by Gratkowski and Storzer, a decreased beta-band activity was observed in the STN from DBS leads. A similar beta decrease was also detected using scalp EEG from parietal and occipital areas [97]. Later, Storzer et al. observed a reduction of beta power in the STN during cycling compared to walking in both PDFoG+ and PDFoG- groups during the OFF state. Additionally, an $18 \mathrm{~Hz}$ power increase was observed in PDFoG+. The low beta-band activity was considered as either a signature of the affected BG or as a movement-inhibiting signal in the motor network [138].

Lastly, as FoG is an event that is mostly triggered during actual walking acquiring subcortical neural biomarkers during active gait paradigms is paramount. During their active gait paradigm, Syrkin-NIkolau et al. compared stepping in place, forward walking, and instrumented walking, which included a turning and barrier course. During stepping in place, a decreased STN beta band power was found in the PDFoG+ compared to the PDFoG-. Additionally, an increased beta sample entropy, which is a measure of unpredictability, was found in PDFoG+ compared to the PDFoG-. During FoG events, increased alpha sample entropy and decreased beta power was found compared to walking 
without FoG. The increased sample entropy in the $\mathrm{PDFoG}+$ group indicated an increase in the error of the processing of sensorimotor information in the subthalamic outflow. On the other hand, the pattern of low beta power and high beta entropy may be caused by a compensatory mechanism adopted to overcome abnormal gait [41]. In addition to cued active gait paradigms, dual-task protocols have also been utilized to explore subcortical biomarkers of FoG. In their work, Chen et al. recorded local field potentials (LFP) from the STN while subjects performed either single or dual-task walking. In this study, FoG vulnerability was derived from accelerometers located at the subjects' trunks. Their results showed an increased LFP power in the low beta and theta bands irrespective of single or dual-task walking. The increased power in the low beta and theta bands were found to be associated with FoG vulnerability. Their findings further support the second mechanism hypothesis, stating that cross-talk between motor, cognitive, and limbic circuits results in synchronous firing from the BG resulting in FoG events [40], [69].

While electrophysiological studies provide an excellent route to capture dynamic loss in cortical connectivity with freezing in PD, electrical measures provide a limited understanding of the cortical-subcortical connectivity and whether there are homogeneous losses in brain structure within PDFoG+. PET and MRI provide a means to study the loss of brain structure in PDFoG+. These neuroimaging techniques have been extensively utilized to understand the pathophysiological mechanisms of PDFoG+ [47], [48], [139]. Converging evidence from these neuroimaging techniques suggests that subthalamic locomotor regions, cerebellar locomotor regions, and mesencephalic locomotor regions may be involved with FoG. This review is focused on the electrophysiological findings 
related to $\mathrm{PDFoG}+$, in which the studies of imaging findings are excluded. However, excellent reviews on imaging technology can be found in recent papers [47], [48], [139].

\subsection{Summary of Findings}

\subsubsection{Differences in EEG Between PDFoG+ and PDFoG-}

Table 1. summarizes the studies that focused on differentiating PDFoG + vs PDFoG- for developing potential biomarkers. For PDFoG+ the LRP in EEG was shown to have an earlier onset and a larger magnitude during cognitive decision making of target detection compared to PDFoG-. These findings support the second mechanistic hypothesis, which states that dual-task triggered FoG events are caused by the loss of automatic updating of motor programs by the dysfunctional BG [39]. In terms of EEG frequency band analysis, the beta band was shown to increase in the frontal region during lower limb pedaling in PDFoG + , indicating preparatory adjustments and motor plan execution through top-down signaling [140]. Also, for PDFoG+ theta band power was attenuated in the mid-frontal location, indicating an impairment in cognitive control [119]. In the study that used the dual-task oddball paradigm, although the stimulus was detected by an increased P300, beta ERD was unchanged for PDFoG + . The inability of $\mathrm{PDFoG}+$ to react to stimuli might have been caused by impairments at the BG. The BG impairments were suggested to either be caused by reduced input to the sensory-motor cortex or by the hypersynchronized thalamocortical activity. Additionally, the duration of beta ERS was found to be longer after the cue signal in the PDFoG+ compared with PDFoG- [128].

\subsubsection{Difference STN Activity Between PDFoG+ and PDFoG-}

When delving into subcortical studies and LFP, an increased high-beta (21-35 Hz) power was observed in the STN during the OFF state in PDFoG+. Levodopa administration 
was found to effectively reduce the high-beta power [37]. In the study on the power coherence between the STN and EEG, the beta burst's duration was longer in PDFoG+ compared with PDFoG- during stepping in place and walking forward [134]. In the bicycling and walking study, the greatest beta band decrease was observed during cycling, indicating its potential therapeutic effect by matching the effects of DBS therapy [138]. Finally, beta power was decreased during stepping without FoG and was proposed to indicate a compensatory mechanism adopted to overcome abnormal gait in PDFoG + . This study also explored sample entropy and found it to be at its greatest during forward walking. This indicated an error in the processing of subthalamic outflow, resulting in abnormal gait patterns [41]. The vast variety of biomarkers and competing results are suggestive of the need for future research to follow similar paradigm and evaluation procedures. For findings to be significant, future research needs to agree on the way bands are segmented, i.e. beta into high and low bands. Also, to more closely match potential FoG event occurrences, more work is needed to focus on actual lower limb task paradigms and the execution of protocols during ON vs OFF medication states.

Table 1. Results and interpretation of studies that compared neural biomarkers of PDFoG+ vs PDFoG-.

\begin{tabular}{|c|c|c|c|c|}
\hline TITLE & PROTOCOL & METRIC & FINDINGS & $\begin{array}{l}\text { INTERPRETATION } \\
\text { (HYPOTHESIS } \\
\text { SUPPORTED) }\end{array}$ \\
\hline $\begin{array}{l}\text { MOTOR } \\
\text { PREPARATION } \\
\text { RATHER THAN } \\
\text { DECISION- }\end{array}$ & $\begin{array}{ll}\text { Detection } & \text { of } \\
\text { differences } & \text { in } \\
\text { cognitive } & \\
\text { decision } & \end{array}$ & LRP & $\begin{array}{l}\text { Earlier onset } \\
\text { in PDFoG+ } \\
\text { compared } \\
\text { with PDFoG- }\end{array}$ & $\begin{array}{l}\text { Excessive recruitment of } \\
\text { lateral premotor areas } \\
\text { due to loss of automatic } \\
\text { motor control }\left(2^{\text {nd }}\right)\end{array}$ \\
\hline
\end{tabular}




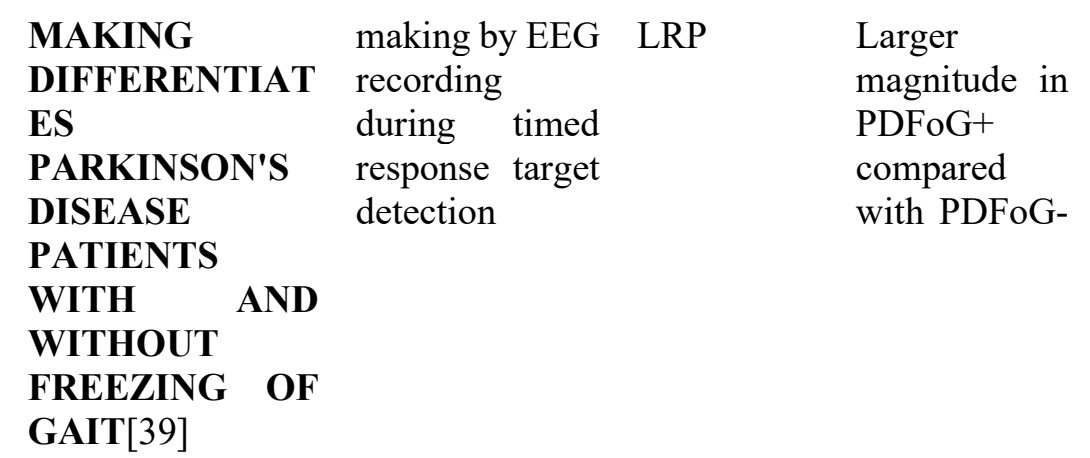

\section{FRONTAL \\ THETA \\ BETA \\ OSCILLATION \\ S DURING \\ LOWER-LIMB \\ MOVEMENT IN \\ PARKINSON'S \\ DISEASE[141]}

EEG recording during lower limb pedaling
Theta (4-8)

Power

(n)

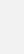

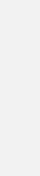




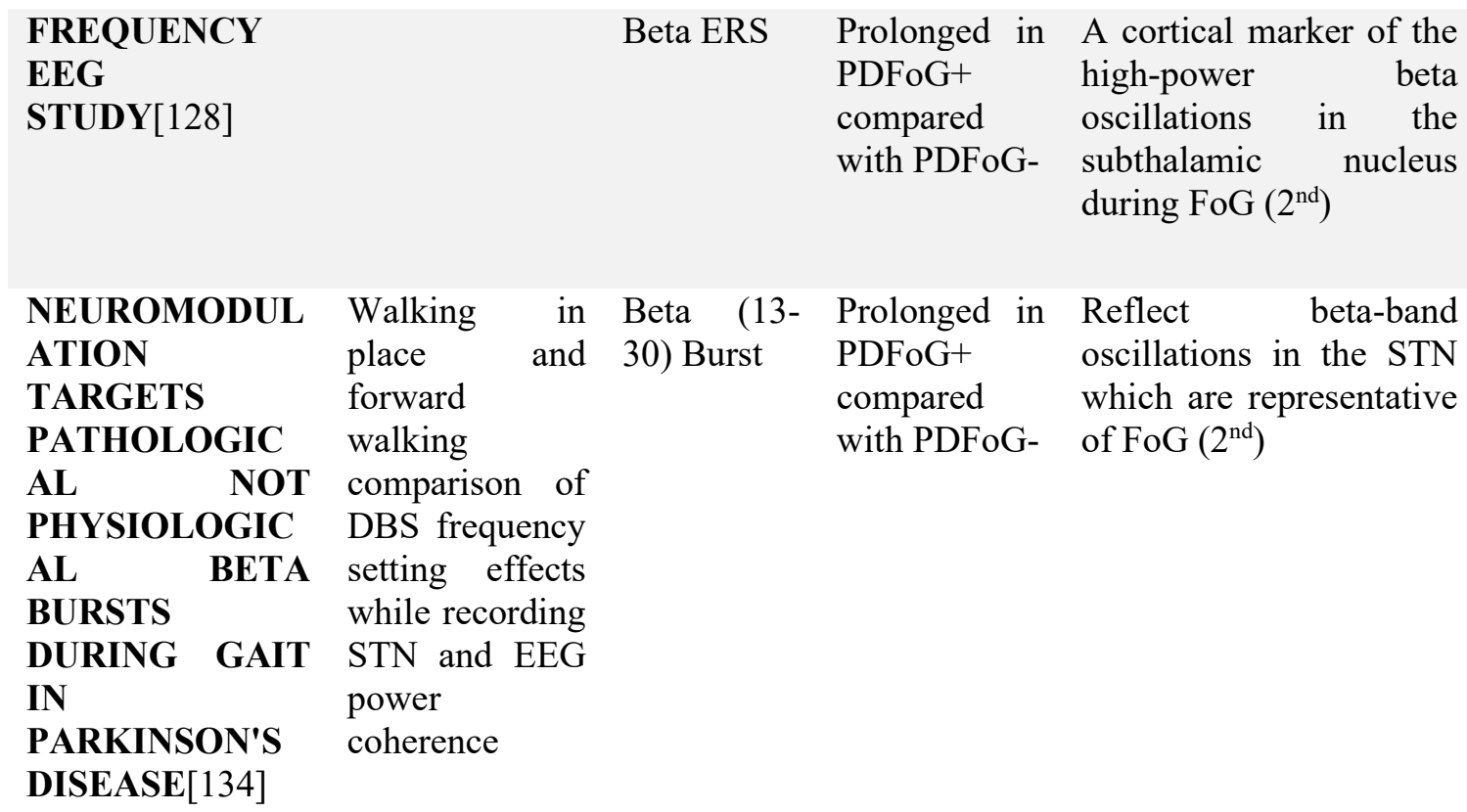

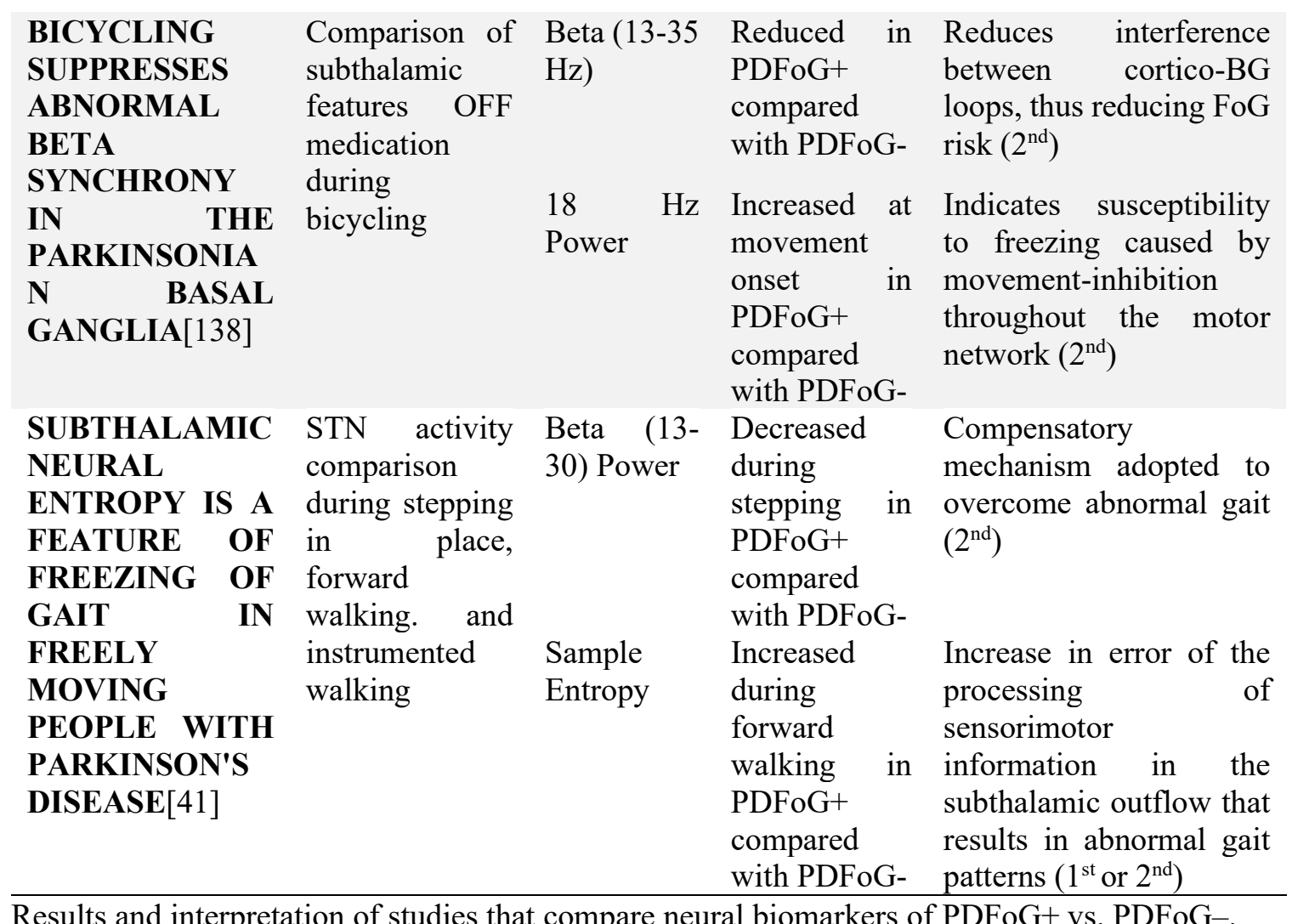




\subsubsection{Difference in the Electrophysiological Activity Between ON and OFF Medication}

From the 2 studies resulting from the search query, one examined the effects of parkinsonian medication. The results from such explorations can even be used to support some of the hypotheses by evaluating the known effect of the medication and the symptom alleviation provided. For example, high-beta power reduction was shown to be mediated by levodopa administration resulting in FoG cessation. This high-beta power reduction resulted in similar oscillatory high-beta levels between the PDFoG + and PDFoGgroups[37]. This finding supports the fifth mechanistic hypothesis, which states that FoG events are caused by a malfunction of the frontal cortex-BG networks via the motor and associative STN loops. This study also found high-beta coherence between the STN and the SMA, which supports the association between the hyperdirect pathway and FoG event triggering. However, dopaminergic medications are not effective for all who develop FoG and may even trigger FoG events, as in PDFoG+ who experience FoG even ON-levodopa. Recent studies have shown that dopaminergic medication improves gait speed, stride length, and reduces freezing events but does not improve gait asymmetry and gait arrhythmicity[19], [142], [143]. Premovement EEG beta desynchronization is reduced in PDFoG+, and this abnormality is at least partially mitigated by dopaminergic stimulation[11], [73], [143]. Additionally, cholinergic loss in the pedunculopontine nucleus may play a role in FoG, as it stands at the crossroads between supraspinal and spinal gait centers[73]. fMRI studies to understand the effects of dopamine on corticalsubcortical connectivity within PDFoG + is an active area of research[144] and may be able 
to distinguish cortical-subcortical connectivity changes between dopamine responsive PDFoG+ and dopamine non-responsive PDFoG + .

\subsection{FoG Event Detection}

In addition to the application of biomarker research, EEG may also be used to detect and even predict FoG events. From the search query, the earliest works employed four channels of wireless EEG systems for FoG event detection [125]. In this work, the wavelet decomposition method was used because of its adaptable and adjustable characteristics that allow for time-frequency localization, multiscale zooming, and multi-rate filtering [125]. A three-layer backpropagation neural network was used with the highest classification accuracy of $76.6 \%$ while relying only on the $\mathrm{P} 4$ channel. The success rate of classification between normal and freezing onset implied that the FoG event could be detected from the neural signature as far back as 5 seconds before the physical representation. This work was further advanced to incorporate spatial, spectral, and temporal features as well as a kNearest neighbor classifier to bump classification accuracy to $80 \%$ [53] then $87 \%$ [124]. The electrodes detected to be most sensitive to the transition of freezing, namely $\mathrm{P} 4$ and $\mathrm{Cz}$, support the finding that freezing impacts the medial parietal areas, which are in charge of integrating sensory information and visuospatial processing [145]. Other FoG event detection studies relied on effective brain connectivity to boost classification accuracy by up to $94.8 \%$ [126]. In this study, findings suggest that hypersynchronization is generated by the frontal region, which is critical for spatial attention, motor intention, cognition, and decision-making processes. These results support the fifth hypothesis underlying FoG, which states that FoG is a consequence of frontal executive dysfunction, where there is a disconnect between the $\mathrm{BG}$ and the frontal lobe, resulting in poor performance when trying 
to achieve multiple tasks [18], [54], [68]. Later studies have also focused on the electrode placement, suggesting an optimal montage of 2 channels, located at C4 and O2 [146]. Meanwhile, others have focused on turning freezing detection (TF), which is a subtype of FoG, to improve detection classification from $68.6 \%$ [116] to $86.2 \%$ [147]. The latest of these studies focused on expanding on the feasibility and robustness of FoG detection with EEG by examining data from more subjects, resulting in a sensitivity and specificity of $82.7 \%$ and $86.6 \%$ respectively [148]. These studies concluded that FoG can be detected and even predicted through the underlying EEG signature with high certainty and a low EEG channel count.

\subsection{Conclusion}

The main aim of this review was to highlight the most recent mechanisms underlying FoG in PD through electrophysiological explorations. The results from these cortical and subcortical level studies can be used to enhance the understanding of PD pathophysiology. Additionally, the high temporal resolution of EEG may be combined with the high spatial resolution of other modalities to understand subject-level cortical-subcortical connectivity in PDFoG+, which may be further combined with diffusion and structural MRI, to understand if there is any backbone anatomical network[149] within PDFoG+ attributing to freezing in such patients. These multimodal approaches may be better able to characterize structural and functional abnormalities within $\mathrm{PDFoG}+$ at an individual level and may pave the way for the identification of potential therapeutic targets to alleviate PDFoG+. From the studies discussed, most FoG exploration studies relied on gait surrogate paradigms due to instrumentation limitations or resultant motion artefacts from active gait. The application of state-of-the-art wearable medical devices will allow for unconstrained 
paradigms with true-to-life triggers and settings[150], which will bring an enhanced understating of PD pathology. Additionally, the combination of real-time neural recordings along with physiological sensing and stimulation will permit closed-loop systems to detect, correct, and even predict FoG events. Future works should then focus on implementing active gait paradigms along with real-life settings to detect actual FoG events. Top-down sensor implementation will then allow for biomarker detection and verification by neural signature confirmation, as biomarkers must relate not only to the resultant physiological response but also to the neuronal mechanism in charge of motor control. 


\section{CHAPTER 3 THE SENSOR APPROACH}

\subsection{Introduction}

Over a century ago, Richard Caton was the first to develop a visualization of the electrical current measured by positioning electrodes at the surface of a skull. About 45 years after this initial discovery, Hans Berger started what is the basis for modern EEG. His very early findings of alpha waves, sleep spindles, and even early works on epilepsy, make Berger the father of EEG [151]. To this day, although the acquisition of EEG waveforms has greatly improved over the century since its birth, electrode performance still proves to be the most challenging aspect to provide a good quality signal. Although neural features have been explored for the detection of biomarkers, non-invasive neural exploration during active gait still proves difficult. This difficulty can be attributed to four challenges: movement artefacts associated with active gait paradigms, the standard sensing systems' cancellation of relevant signals, the inability to achieve volition due to comfort, and multi-sensory co-registration of wearable sensors. These challenges are augmented in PD patients who may exhibit tremors, dyskinesia, and other symptoms, further adding to the motion-corrupting artefacts during active gait. Additionally, due to cognitive decline, PD patients may not be able to sustain long periods of activity, making long recordings unfeasible. To successfully capture the neural patterns associated with active gait, the sensors used for exploration must; be synchronized with each other to co-register neural and muscular activations; have a recording bandwidth that is sensitive to the features of cortical potentials; be unobtrusive to ensure executive motor control is not affected by the sensing system; be ultra-wearable and easy to set up; and allow for movement artefact attenuation to guarantee signal quality. 
The top-most sensor in the proposed suite is the EEG, able to record electrical potentials from the scalp. The EEG records neural activity in the range of microvolts and is thus highly sensitive to motion artefacts. The two main sources of noise in EEG recordings are stationary noise whose statistical parameters do not change in time, these include white noise and sinusoidal noise from sources such as power outlets. The second source of noise in EEG recordings is non-stationary noise, whose statistical parameters do change with time. This noise can be composed of eye blinks, pulmonary and cardiac activity, and movement artefacts from physical motion, such as active gait. As this non-stationary noise is more difficult to filter out, three common methods are employed to deal with these artefacts: artefact-free sampling, repeated recordings, and the use of hardware designed to deal with active recordings. Artefact-free recordings are achieved when noisy segments are removed manually or when one co-register to motion sensors and/or behavioral data to detect corrupted segments. Repeated recordings may also be employed to rid data of artefacts. Considering that stationary noise can be simply filtered out, non-stationary noise may be averaged out over enough recordings. This approach is common practice when recording MRCP, where typical protocols call for trials repeated 40 to 300 times then averaged. Lastly, hardware designed specifically for the task of active gait recording may be used to guarantee artefact-free neural signals. To this effect, several groups and companies have created proprietary versions of wearable EEG systems. However, some of these devices are not built to allow for active gait recordings, result in problematic setups, and high levels of noise [152], [153], or are designed with built-in filters that attenuate relevant signals in very low-frequency ranges [154]. Additionally, for the active gait neural exploration, start and stop activity must be synchronized with the EEG activity. 


\subsection{Background}

To this day, the interface between the scalp and the electrode still proves challenging to set up properly. This is due to the insulatory characteristics of the scalp which holds oil and hair, not to mention the skull itself which acts as a low pass filter (Srinivasan 98). Common EEG practice dictates the removal of surface oil and dead skin cells by rubbing with mild abrasives [155]. This process is then followed by the insertion of an electrolyte gel, commonly made of sodium and chloride, which merges the gap between the metallic electrode and the surface of the scalp, helping to reduce the impedance down to the acceptable $5 \mathrm{KOhm}$ for individual electrodes and $1 \mathrm{KOhm}$ between electrodes [155]. This electrolyte also acts as a vibratory dampener by allowing the free flow of ions even when the electrode tip is not in direct contact with the scalp. This interaction results in an electric double layer, which is made by the lining up of opposing charges between the electrolyte and the metallic electrode, increasing capacitance and improving the flow of current created by the electrical fields under the skull [151]. However, with applications requiring an increasing number of electrodes, the preparation and setup of gold standard devices can take a single experimenter 40 minutes for a 64-channel wet electrode system [156]. Also, because the electrolyte is generally water-based, it dries out within a couple of hours of setup and is also susceptible to dilution by sweat, thus reducing its main conductive benefits.

The long setup required, susceptibility to signal quality decrease over time, and discomfort to the user have led to a shift towards "dry" electrodes. These are commonly made of similarly inert metals such as gold, or of silver-silver chloride electrode-covered 
tips. However, unlike wet electrodes, these rely merely on mechanical contact with the scalp to provide the necessary interface for the flow of current.

\subsection{Proposed Solution}

For the exploration of neural activity during active gait, reliable noise-free data is needed. To solve the sensor approach challenges the following requirements were established: the suite would have been able to detect neural features during active gait, it had to be wearable and not interfere with normal function, and all the sensors within the suite needed to be time-synchronized. This study proposes a whole-body suite to encompass the EEG activity from the head recorded at the surface of the skull, Figure $2 \mathrm{a}$, $2 \mathrm{~b}$, and $2 \mathrm{c}$. Alongside EEG, the electrical activity from the muscles involved in gait control Figure $2 \mathrm{~d}$, as well as the kinetic and kinematic features associated with the gait phases during locomotion, Figure 2e. Additionally, following Stuart's recent review on cortical

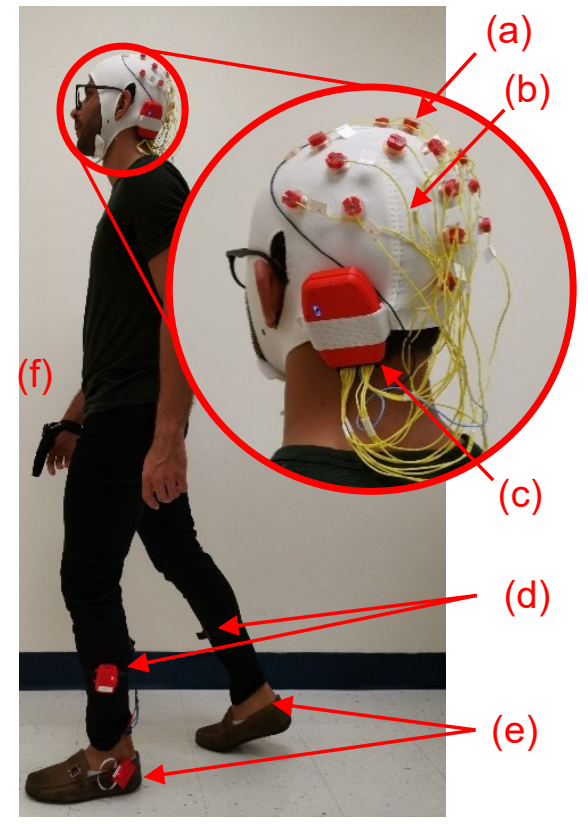
activity identifiers for the elder and those PwP, the relationship between the neural activity and behavioral outcomes should also be consistently recorded and reported [51], to achieve behavioral tracking, an upper limb device, which records bradykinesia using an angle sensor at the perlicue, was designed and tested for clinical use as a companion to objectively assess disease progression, Figure $2 \mathrm{f}$.

Figure 2. The complete sensor suite, custom-made for active gait neural exploration. (a) The custom wet $\mathrm{Ag}-\mathrm{AgCl}$ electrodes with low noise, (b) EEG electrode array with short cabling for artefact reduction, (c) wearable EEG sensor with wireless connectivity; (d) electrical activity from the muscles; (e) kinetic and kinematic activity from gait; (f) behavioral and symptom tracking. 
Successful neural activity exploration is contingent on robust and reliable synchronicity between the EEG, EMG, GRF, and upper limb symptom analysis devices. The following section will cover the devices that make up the ultra-wearable suite. Each subsection will encompass each of the devices and the specifications that warrant its inclusion into the proposed suite.

\subsection{Sensors Development}

\subsubsection{Wearable EMG}

Figure 3. shows the EMG system which will synchronously record electrical potential from the surface of the skin. For this, a lightweight, low-power consumption, batterypowered, and wireless-enabled sensor, using an ADS1292 chip, will be employed. The analog-to-digital converter ADS-1292 module is a low-noise 24-bit analog front-end biopotential measurement system by Texas Instruments (Dallas, TX, USA). A 32-bit MPU (CC3200) with built-in Wi-Fi connectivity was employed for on-board real-time signal processing and data transmission. A high-precision EMG signal allows for less than 0.8 $\mu \mathrm{V}$ of peak-to-peak noise.

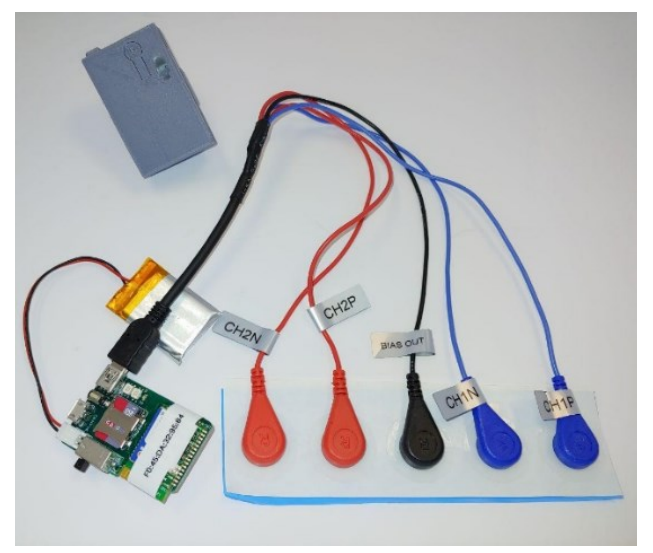

Figure 3. EMG system with DAQ and electrodes. 
The custom-made CyberSens device was designed to provide a seamless recording and the transmission of two channels of a 24-bit EMG signal, with the sampling rate up to 8000 Hz. The wireless transmission can also reach 100 feet in an open space. Likewise, an MPU9250, which meets the requirement for studying human locomotion, was employed for kinematics and noise estimation. This device has been thoroughly tested in several studies from our group, with as many as ten individual sensors wirelessly connected, supplying synchronous muscular activity information for live active-prosthetic tuning and energy expenditure optimization [157], [158].

\subsubsection{Channel EEG}

Following Lopez-Gordo's recommendation for electrode description and evaluation the following sensor characteristics needed to be assessed or ensured for the wearable EEG data acquisition system (DAQ) and accompanying electrodes. First an Impedance between electrodes in the range of 5 to $40 \mathrm{~K}$ ohms (Typical of $5.6 \mathrm{Kohm}$ ). A valid frequency range along the relevant neural bands. Noise level characteristics and a biosignal application procedural feature measure.
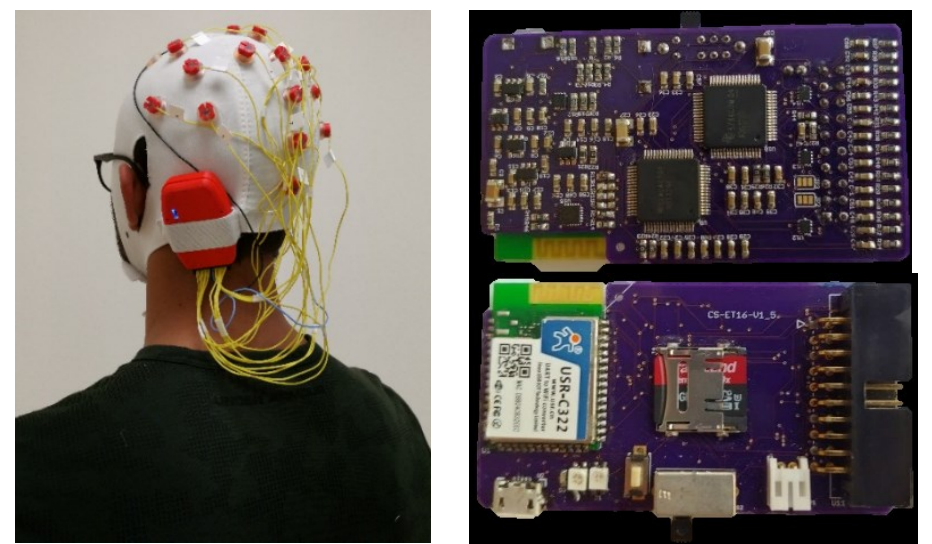

Figure 4. The EEG system. (Left) 16 channel EEG system mounted on a custom EEG cap. (Right) The top image shows the EEG DAQ with 2 ADS1299s, power management, and embedded IMU. The bottom image shows the CC3200 for WiFi connectivity and onboard storage. 
At the core of the EEG DAQ is the microcontroller, Figure 4. For this, on-board realtime signal processing, and wireless data transmission, the 32-bit CC3200 multi-core processing unit, capable of WiFi connectivity, was used. This device provides seamless recording and transmission of sixteen channels of 24-bit EEG signal with a sampling rate of up to $1000 \mathrm{~Hz}$ per channel. The range of the wireless transmission can reach about 100 feet in an open space, allowing for natural walking made of voluntary starting and stopping.

For digital to analog conversion, two ADS1299s were used in cascade mode. This configuration is beneficial because although it requires more general-purpose input/output pins than the Daisy-chain mode, multiple readback mode is sustained. Benefits of the ADS1299 (Dallas, TX, USA) include a low required minimum signal bandwidth, of 10$\mu \mathrm{V}_{\mathrm{Pk}}$, an input impedance of more than $10 \mathrm{M} \Omega$, lead-Off detection, programmable gains, and an input-referred noise of $1.0 \mu \mathrm{V}$ peak-to-peak. In a dorsiflexion and active stepping experiment, by Rashid et al. A comparison was made between the ADS1299 and the NuAMPS gold standard EEG system. Comparable signal quality was found for the premovement noise, which represents the EEG activity from 2-3 s before the EMG onset, regarded as a baseline in $\mathrm{MRCP}$ and $\mathrm{ERD} / \mathrm{S}$ patterns. Also, comparable signal-to-noise ratios (SNR) were found during both tasks. In this study, the SNR was defined as the ratio of peak negative amplitude to the root mean square value in the baseline segment of the averaged MRCP, expressed in decibels [159]. This comparison study functioned as validation for the use of the ADS1299 in our wearable suite.

Further, an inertial measurement unit (IMU) was also embedded in the EEG system. The MPU 6050 (gyroscope + accelerometer+ magnetometer) micro-electro-mechanical system by InvenSense was employed for kinematics data acquisition. This chip provides a 
user-programmable gyroscope full-scale range from \pm 250 , to $\pm 2,000^{\circ} / \mathrm{s}$ and a userprogrammable accelerometer full-scale range from \pm 2 to $\pm 16 \mathrm{~g}$, which meets the requirement for studying human locomotion. An eight-channel version of this DAQ was originally developed by our group and has been tested in-depth [56]. The 16-channel DAQ was designed and built to be used with two sets of in-house built electrodes, dry and wet.

\subsubsection{EEG Electrode}

Unlike wet electrodes, dry electrodes are easy to mount and have been shown to be preferred by the wearer over wet electrodes [160]. Methods to provide dry electrodes have resulted in several electrode types; gold-coated single pin, multiple pins, and solid-gel electrodes [161]. In Di Flumeri's comparison, comparable mental state classification and signals spectra values were found between these three dry electrode types and the wet gold standard. However, these electrodes were used with proprietary DAQ, this adds a variable to be considered in their comparison and limits their implementations to sensing systems that prove as expensive as the wet gold standard [161]. Not only does the inherent design of the electrodes require specific DAQs, but also, because there is no electrolyte, a constant applied pressure is required, this may lead to discomfort over long recordings overcoming the benefit of not using an electrolyte [162], [163]. Finally, these dry electrodes are sensitive to surrounding noise from persons and other electrical equipment [164].

Others have also developed dry electrodes made of micro-needle tips, spring tips, and conductive polymers [165]. Commercially available examples include those by BrainProducts, who make a single gold-pin electrode with three variable pin sizes that are individually sprung, g.Tec, who makes 2 8-pin fixed-length active electrode sets, similarly Quasar who created an electrode with two sets of rings with spikes and integrated amplifier, 
licensed by Wearable Sensing, and the dry-solid gel-based electrodes also made by BrainProducts. As discussed, these require independent DAQs and are then bound to proprietary recording systems. Recently Xing developed and tested a graphene-based polymer system, which allowed for good SNR and classification accuracy of steady-state visual evoked potentials (SSVEP). However, the testing was made in the range of 0.1 to $1000 \mathrm{~Hz}$, which although covers a large bandwidth [165], does not span to the lower frequencies required for motor execution preceding features such as in MRCP that lie in the 0.05 to $5 \mathrm{~Hz}$ range [166].

\subsubsection{Electrode Development}

This study presented a dry EEG sensor with similar quality to the standard wet electrodes, designed to be interfaced with any other open-source DAQ. Comparable signal quality to wet electrodes is proposed by applying a mechanical springing mechanism, as well as, an embedded Faraday cage used to insulate from electrical interference and motion-related noise.
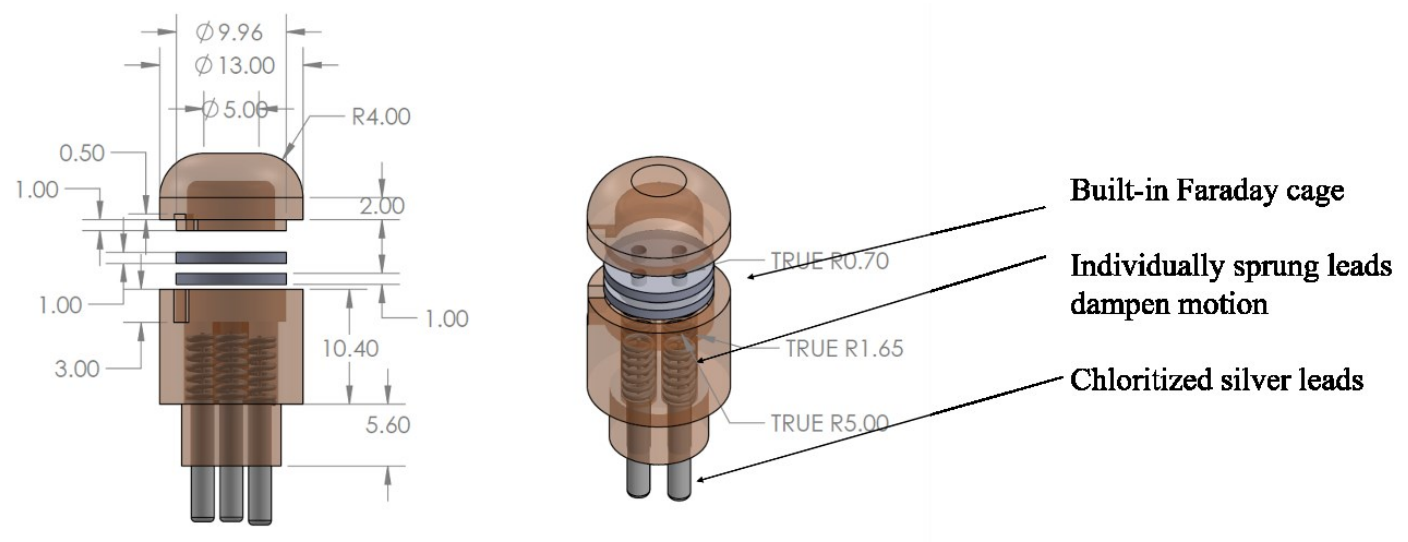

Figure 5. Four lead, individually sprung dry EEG electrode design. The built-in Faraday cage allows for outside electrical interference to be isolated, while the spring mechanism allows for dampening of motion during high activity recordings. All measurements are in $\mathrm{mm}$. 
This mechanism will also act as a dampener that will provide comfort to the wearer. Shown in Figure 5 is the design of the electrode, which was made using SOLIDWORKS 2018. The electrode consisted of four individually sprung 12 gauge .925 sterling silver rods that were chlorinated using the methods suggested by Warner Instruments [167]. Having four individually sprung contact points allows for constant mechanical contact with the scalp even under motion and as reported by Symeonidou et al. who found that increasing the surface area of the electrode has a significant effect on improving EEG signal quality [168]. Each rod had a conical head with a $0.4 \mathrm{~mm}$ radius and measured $11 \mathrm{~mm}$ from the tip of the hemisphere that contacted the scalp to the contact at the back where a silver wire was soldered. The length of the rods was determined by the compression of the springs and the width of the case that held each silver rod and spring mechanism. This allowed for a maximum length of $7.25 \mathrm{~mm}$ and a minimum of $3 \mathrm{~mm}$ under full compression. Following Hooke's law, $F_{\text {spring }}=-k x$, each silver rod generated a maximum normal force with the scalp of $0.618 \mathrm{~N}$, given that the spring used had a spring constant of $145.355 \mathrm{~N} / \mathrm{m}$ and the spring was compressed by a maximum displacement of $0.00425 \mathrm{~m}$. For this study, several springs with differing springs coefficients and lengths were evaluated. A spring with a free length of $15.75 \mathrm{~mm}$ and the above-mentioned spring coefficient was used as it resulted in a compromise between the wearer's comfort and a dynamic movement that allowed the electrode case to be rotated up to 55 degrees. This electrode was designed to be mounted on the sites of the International standard 10-20 system using a standard EEG cap.

The case that houses the silver rods and the springing mechanism was designed to be 3D printable, this lowers cost and allows for rapid changes to be made and implemented, Figure 6. 


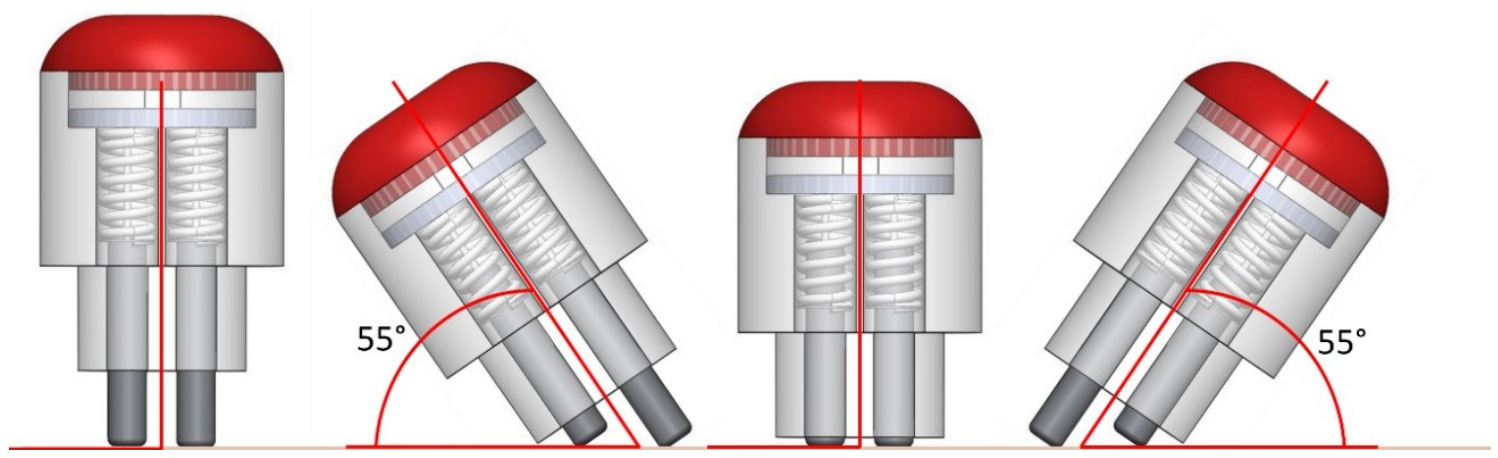

Figure 6. Dry EEG electrode range of motion. The individually sprung mechanism allows the silver springs to be exposed up to $7.25 \mathrm{~mm}$ from the case during minimal compression and up to $3 \mathrm{~mm}$ at full compression. Having four individually sprung silver rods allows for constant contact with the scalp.

To reduce the impact of external interference, a Faraday cage was designed to be embedded into each electrode case. The cage is made of a thermoplastic composite with copper strands. The chromium zirconium copper alloy has great thermal and electrical conductivity, thus reducing external interference and functioning as a thermal sink to reduce the thermoelectric effect, which is known to change the electrical conductive characteristics of electrodes [169]. The mechanical aspects have already been described, these include fundamental physical attributes, materials used, dimensions, and fixation to the scalp. In terms of electrical attributes, the electrodes proposed will not rely on embedded amplification.

Due to the high SNR requirement of cortical recorded neural activity and the need to attenuate motion artefacts through custom short cabling. Custom wet EEG electrodes were also designed and tested to be used with the custom 16-channel EEG DAQ. Like the EEG dry electrode, the EEG wet electrode was designed using SOLIDWORKS 2018. After several rounds of design and testing, a final solution was selected to be made of 4 parts: the body and sensor cap shown in Figure 7, designed to be 3D printed and easily modified, and a pair of 12 gauge .925 sterling silver rods that were chlorinated using the methods 
suggested by Warner Instruments [167]. This in-house built EEG wet electrode was tested using an MRCP evaluation, which was acquired using the complete ultra-wearable solution and will be discussed in section 3.5.

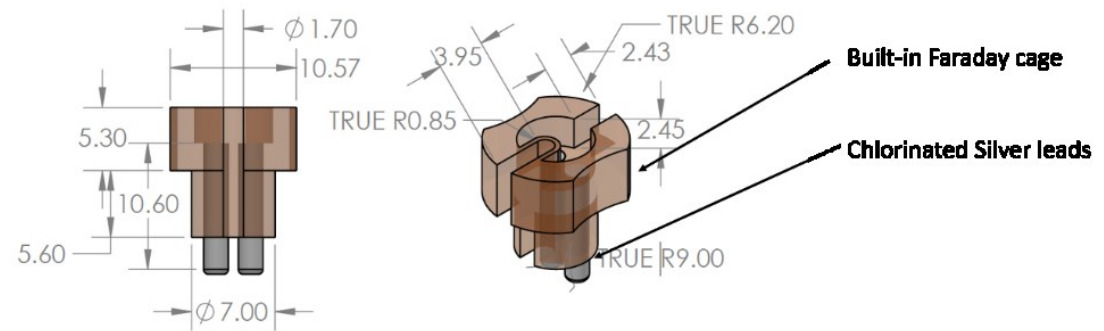

Figure 7. Two lead, wet EEG electrode. The built-in Faraday cage allows for outside electrical interference to be isolated, the electrolyte used dampens motion and functions as an electrical double layer between scalp and electrode. All measurements are in $\mathrm{mm}$.

\subsubsection{Preliminary Dry Electrode Testing}

Standard EEG procedure dictates maintaining impedance between $5 \mathrm{k}-40 \mathrm{k} \Omega$ This has commonly been done by using an abrasive pad to get rid of oil and sweat. However, this practice is widely being replaced to avoid breaking through the skin. Instead, gold standard EEG systems use saline and provide electrode-by-electrode impedance measurements. However, these cost upwards of 10k USD for a 16-channel EEG device. Instead of adding a circuit to each electrode, which would increase complexity and cost. We used the ADS1299 built-in lead-off function allowing for either DC or AC stimulation. Our device could pin-point which electrode does not meet a pre-defined DC value established during setup. This was done by injecting a known DC through a selected pull-up or down resistor then measuring the output and comparing the difference. DC lead-off detection can only be done during power-up, or during a programming state, because the BIAS amplifier must be powered off. The ADS1299 also possesses an AC signal source with programmable stimulation current and frequency. 

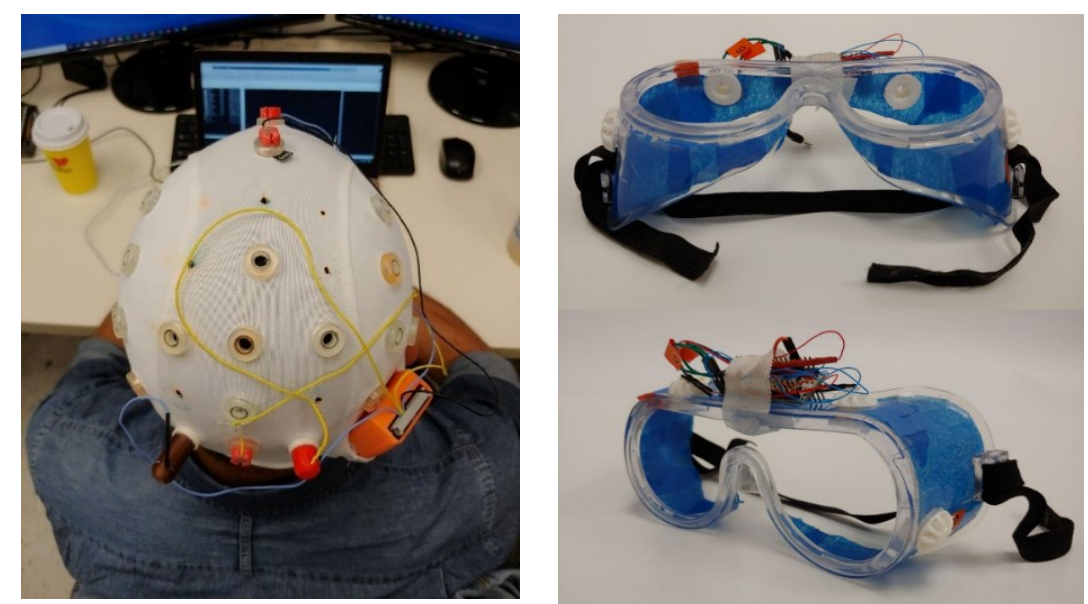

Figure 8. EEG electrode testing setup. (Left) Dry electrode feature SNR setup with embedded Faraday dry electrode at $\mathrm{O} 1$ and standard wet at Oz. Both electrodes are connected to the inhouse developed 16-channel EEG DAQ. (Right) Visual evoked potential stimulator, designed to control the flicker of a set of light-emitting diodes. Flicker was verified using an oscilloscope.

By measuring the magnitude of the output at the excitation signal frequency (usually at $1 / 4$ of sampling frequency), intermittent impedance can be measured to ensure individual channels are kept at the desired 5-40k Ohm impedance range. After ensuring the electrode impedance could be monitored during the setup and the actual recordings. The range of frequencies and noise characteristics needed to be evaluated. As shown in Figure 8 left, while wearing a wet and dry electrode in the occipital region, grounded at Afz and referenced at Fz. One user was asked to perform 10 eye blinks followed by 10 seconds of eyes closed (to generate alpha activity), and finally, the subject was asked to perform jaw clenches for 10 seconds. 


\subsubsection{Preliminary Dry Electrode Results}
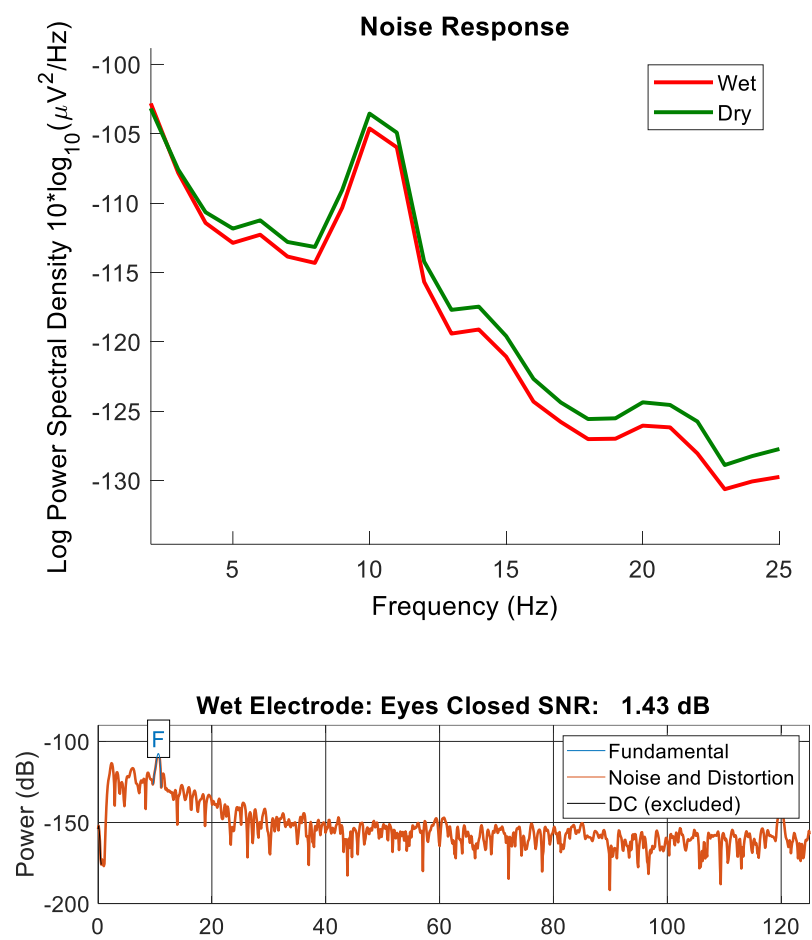

Figure 9. EEG electrode noise and SNR results. (Top) Frequency range characteristics for both wet and dry EEG electrodes during an eyes-closed task. (Bottom) Wet EEG electrode signal-tonoise ratio during the eyes-closed task, expected to elicit alpha activity in the $8-12 \mathrm{~Hz}$ band.

This test resulted in a higher power spectral density for the wet electrode throughout the relevant frequency compared with the dry electrode, Figure 9. Additionally, the wet electrode's 1.43 dB SNR value fell within the SNR range found in the literature [170]. This task-specific test showed that the wet electrode outperformed the dry in both SNR during eye blink and across relevant neural band frequencies. The purpose of this comparison was twofold. First, to show which electrode was least prone to artefacts from electrical interference in a noisy environment. Secondly, to highlight the similarity between the wet and dry electrodes, showing the potential of the dry EEG electrode as a future interface between the scalp and the EEG DAQ, without the need for long to set up and uncomfortable gelling. 
In addition to the range of frequency response and noise comparisons, a procedural neural response with known features needed to be tested using the designed wet and dry EEG electrodes. For evaluating the performance of the proposed dry EEG electrode, a comparison between feature SNRs using SSVEP was carried out. Brain-computer interface systems often rely on neural feedback associated with a stimulus as inputs for control. In the case of SSVEP, a stimulus with known temporal and spectral properties is employed, this is then matched to neural patterns often recorded from the visual processing areas of the brain [171]. To test the performance of the proposed dry EEG electrode, two electrodes were positioned along the occipital lobe Figure 8 Left, following the international standard 10-20 system. A wet electrode was positioned at $\mathrm{Oz}$ and a dry electrode with embedded Faraday cage at O1. For ground and referencing, two wet electrodes were positioned at $\mathrm{AFz}$ and Fz, respectively. The proposed DAQ was used to record each sensor's data, which were individually sampled at $250 \mathrm{~Hz}$. Five trials of continuous stimulation using a $9 \mathrm{~Hz}$ flashing white light was administered to one subject for 20 seconds durations. For preprocessing a $1^{\text {st }}$ order Butterworth bandpass filter was applied between 2 and $20 \mathrm{~Hz}$. The SNR was calculated by dividing the amplitude of the visual evoked potential component at peak latency by the noise power [172]. 


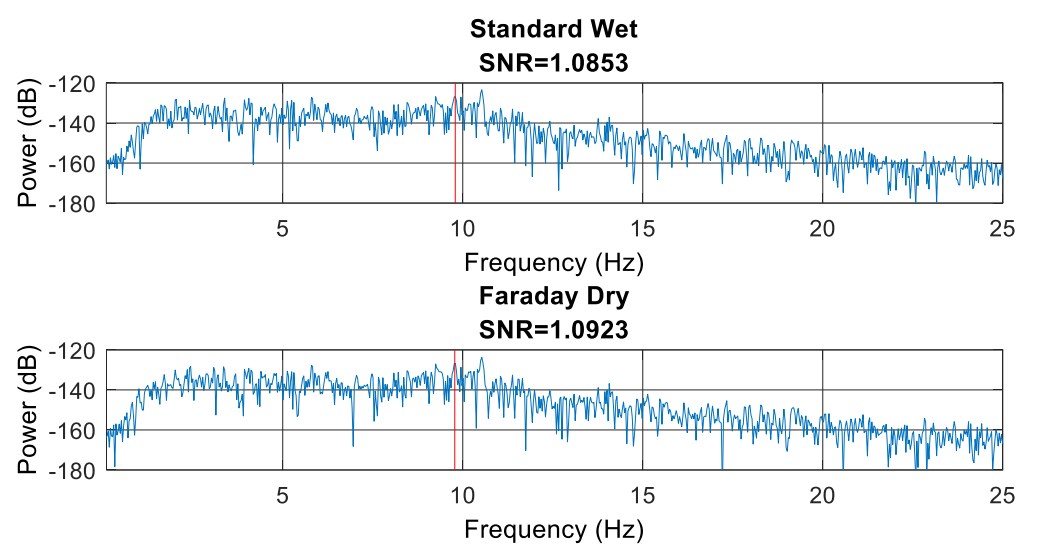

Figure 10. EEG electrode SSVEP results. SSVEP triggered at $9 \mathrm{~Hz}$ showed improved SNR from regular case dry to the standard wet.

As shown in Figure 10, the EEG activity shows a peak at the $9 \mathrm{~Hz}$ stimulation frequency from the visual evoked potential. This peak was of similar magnitude compared with the baseline noise for both standard wet and faraday dry electrodes. The similar SNR values for the SSVEP task using both electrode types show the potential of the proposed dry EEG electrode for recordings in BCI applications. However, to ensure its feasibility for regular EEG recordings, further evaluation works are needed as proposed by Lopez-Gordo [173]. Future works should evaluate other paradigm features in addition to SSVEP. Lastly, usability features should be tested to guarantee comfort and applicability in different head types.

The developed wearable EEG system culminated in a device small enough to be comfortably worn close to the electrode locations. The small size is important because a light and small device can be positioned closer to the head, allowing for short cabling and thus reducing motion artefacts. The simple design allows for set up on the subject's head in 5-10 minutes. This is crucial for its use with persons with neural disorders, as the cognitive impairments associated with disease progression make extended recordings and long setup times, unfeasible. 


\subsubsection{Wearable Ground Reaction Force Sensor}
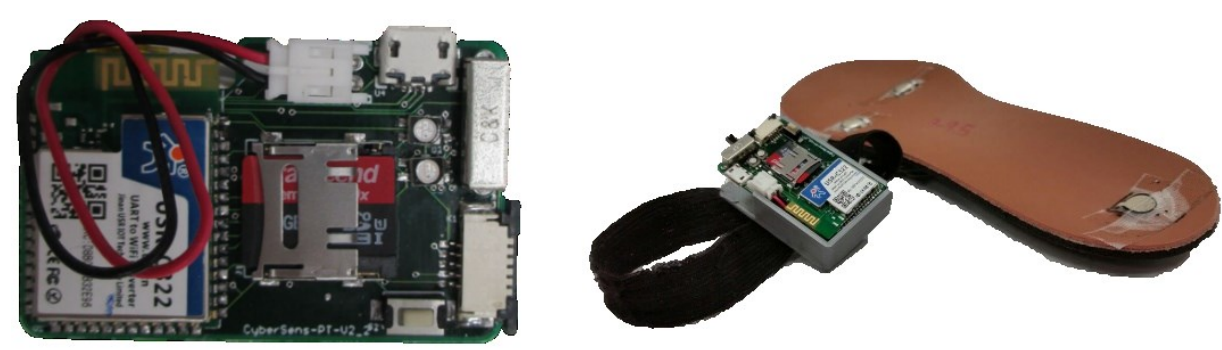

Figure 11. Wearable gait analysis system. (Left) Wireless GRF sensor. (Right) GRF sensor and accompanying insole with 3 piezoresistive transducers.

For gait analysis, a miniaturized system was developed to be comfortably worn at the ankle, Figure 11. The system is capable of wireless connectivity by use of the CC 3200 module. Onboard data storage is also available by employing a micro-SD flashcard. For motion tracking, the MPU-9250 was also used. GRF is measured using Tekscan's A301 piezoresistive transducers. The pressure signal is then amplified using an MCP6004 OpAmp. Each system is comprised of two wireless units that clip on the user's shoe and two insoles with three embedded piezo transducers. This device has been used in leg-length discrepancy detection, active prosthetic tuning, and gait phase segmentation for start-stop prediction based on EEG features [59], [102], [158]. A sample waveform for the kinetic sensors during bipedal locomotion is shown in Figure 12.

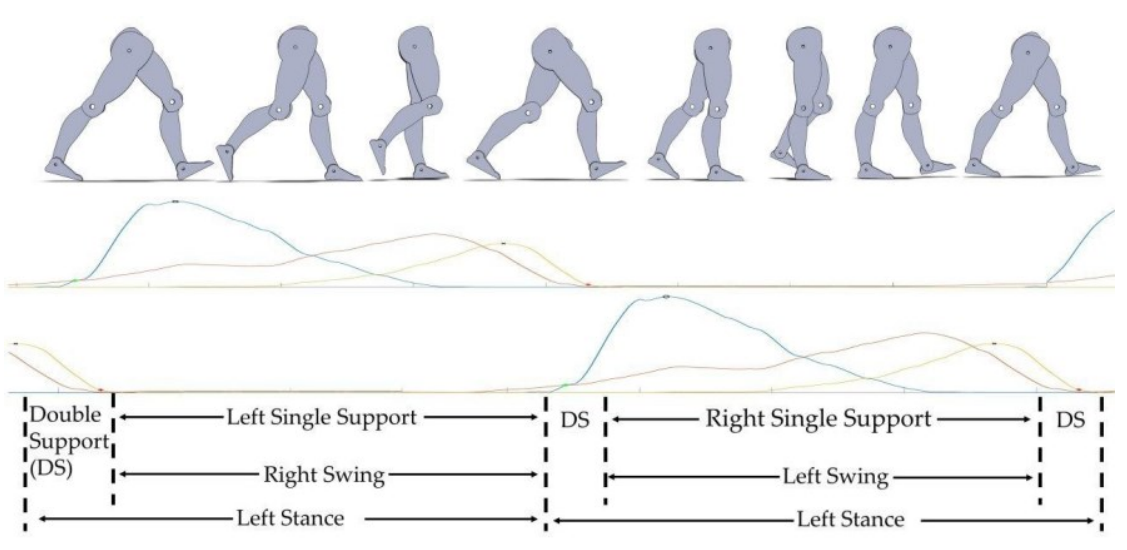

Figure 12. Kinematic gait phases from the wearable ground reaction force sensor. 


\subsubsection{Wearable Symptom Measurement}

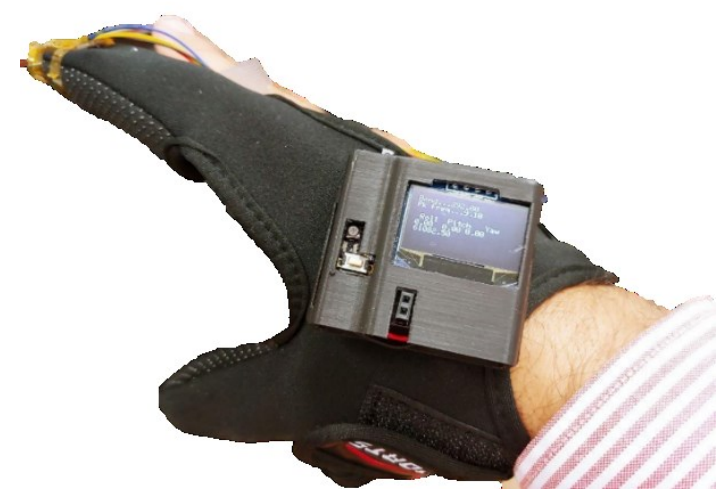

Figure 13. Wearable movement assessment device with internet connectivity. This device is composed of one inertial measurement unit, located at the distal part of the index finger and a flex sensor located at the purlicue.

For objective dyskinesia and bradykinesia capture, an upper limb symptom measurement system will be used, Figure 13. The system is capable of wireless connectivity by the usage of a CC 3200 module. Onboard data storage is also available through a micro-SD flashcard. For motion tracking, an externally mounted MPU-9250 was used. The hand opening and closing are measured using a spectral symbol SEN-08606 flex sensor. Having an objective assessment of disease prognosis will support treatment modification for accurate and timely intervention. Although several studies have successfully explored the feasibility of motor symptom measurements, the common clinical practice still relies upon a subjective assessment performed every 3-6 months, which encompasses finger tapping, pronation and supination, hand opening and closing, and the evaluation of resting, and postural tremors.

\subsubsection{Preliminary Symptom Measurement Testing}

This device was tested as a clinic companion to objectively quantify the movement assessments associated with Parkinson's Disease. Fifteen persons with Parkinson's Disease performed the finger tapping test described in the Movement Disorder Society-Sponsored 
Unified Parkinson's Disease Rating Scale (MDS-UPDRS). While an experienced Specialist in Movement Disorders (SMD) rated each finger tapping test, the device shown in Figure 13 recorded kinematic data from a sensor located at the distal part of the index finger and kinematic features from a flex sensor located at the purlicue. Bradykinesia was calculated as the decrease in opposition of index flexion amplitude from consecutive iterations, as well as, the slowness and irregularity of finger tapping. From these metrics, statistical moments including mean, variance, skewness, and kurtosis, were extracted then min-max normalization was applied to each feature. Fisher's linear discriminant analysis was then computed to lower the dimensional space and ensure separability. A total of thirty movement assessments were performed on fifteen subjects. Based on this dataset, various machine learning algorithms were trained to predict the PD stage as rated by the MDS. Table 2 shows preliminary findings which resulted in prediction accuracy of 95\%, indicating the potential for a standalone device capable of providing real-time objective symptom assessments.

Table 2 Predicted score consensus with SMD rating

$$
\text { SMD's MDS-UPDRS Rating }
$$

\begin{tabular}{c|ccc}
\hline & Zero & One & Two \\
True positive detection & 2 & 4 & 13 \\
False positive detection & 0 & 1 (Labeled as two) & 0 \\
Stage count & 2 & 5 & 13
\end{tabular}

Table 1 Consensus between the SMD's inspection and the algorithm-based classification. Stages Zero and Two were correctly labeled for $100 \%$ of the samples, while stage One had an $80 \%$ true positive detection and a $20 \%$ false-positive detection on stage two due to its larger training size. Other models also yield similarly well-performing classifiers ranging from $80 \%$ combined accuracy for the fine k-nearest neighbors, $85 \%$ for the linear support vector machine, $90 \%$ for fine and medium trees, and $95 \%$ for quadratic support vector machine. 


\subsection{Ultra-Wearable Suite Pilot Recording}

This ecosystem has been developed and tested to achieve the necessary high-quality EEG recordings and co-registration

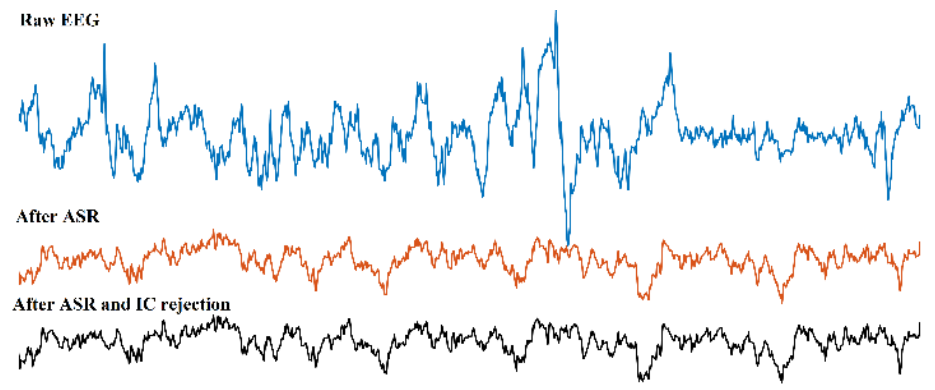
with other suite devices while performing active gait. As

Figure 14. Motion artefact correction. From top to bottom, this figure shows the steps taken to clean the raw EEG data from motion corrupting artefacts.

mentioned before, one of the major issues with non-invasive neural explorations is the problems of synchronicity and noise from artefacts. This study aimed to test the proposed ultra-wearable suit, which encompassed multisensory data acquisition of scalp electrodes for EEG, skin surface electrodes for EMG, and ground reaction sensors for gait phase segmentation. One subject was asked to perform 5 gait cycles from a standstill at a selfselected pace, starting and stopping were also self-timed. A total of 171 events were recorded, from which 155 epochs were extracted. Sixteen EEG channels were recorded and sampled at $250 \mathrm{~Hz}$, at electrode locations: F3, Fc3, F4, Fc4, FCz, C1, C3, C5, C2, C4, C6, $\mathrm{CP} 3, \mathrm{CP} 4, \mathrm{CPz}, \mathrm{Cz}$, and Pz. Epochs were considered as the time 4 seconds before and 2 seconds after gait initiation as marked by the EMG onset for a sensor located at the tibialis anterior (TA), the baseline was selected to be between 4 and 2 seconds before gait initiation. Data were processed using the eeglab toolbox by Delorme \& Makeig on MATLAB [174]. Where it was bandpass filtered between $0.05-10 \mathrm{~Hz}$. Then, sinusoidal artefacts were estimated and removed using the CleanLine function. Afterward, artefact subspace reconstruction (ASR) was applied to remove flatline channels, low-frequency drifts, noisy channels, short-time bursts, and incompletely repaired segments from the data. 
At this point, the recording was re-referenced to the common average. Independent component analysis (ICA), which is a decomposition of input data using the logistic information, was then applied, Figure 14. From start-stop event averaging and the methods mentioned above, noise from active gait was attenuated allowing for non-invasive active gait movement-related cortical potential recordings. Because of the similar frequency response of the MRCP features relative to the baseline signal, the SNR was measured using the methods suggested by Karimi et al. expressed in decibels $\mathrm{dB}$.

$$
S N R=\frac{\sum_{l=1}^{L} \sum_{t=0}^{T}\left[x_{S}^{l}(t)\right]^{2}}{\sum_{l=1}^{L} \sum_{t=0}^{T}\left[x_{N}^{l}(t)\right]^{2}}
$$

where the $l$ th epoch $x_{S}^{l}(t)$ and $x_{N}^{l}(t)$ are the signal and noise epochs, respectively taken from [-4, -2] and [-1, 1] s relative to the onset with $T$ samples and $L$ total epochs [175]. Using previously reported SNR values for MRCP, we set a threshold for success to be above $5.56 \mathrm{~dB}$, as measured using a gold standard $\mathrm{Ag} / \mathrm{AgCl}$ wet electrode [159].

\subsection{Results}

The results from this pilot showed the feasibility of the in-house developed sensor solution for detecting neural patterns during active gait. Figure 15 shows the signature MRCP neural patterns before gait initiation. The potential shift in the cortical activity preceding voluntary movements was co-registered to muscle activity to detect the stimulusresponse. Proving the successful implementation of the biopotentials from the scalp, muscles, and upper and lower limb features. The custom sensor solution could be set-up in 


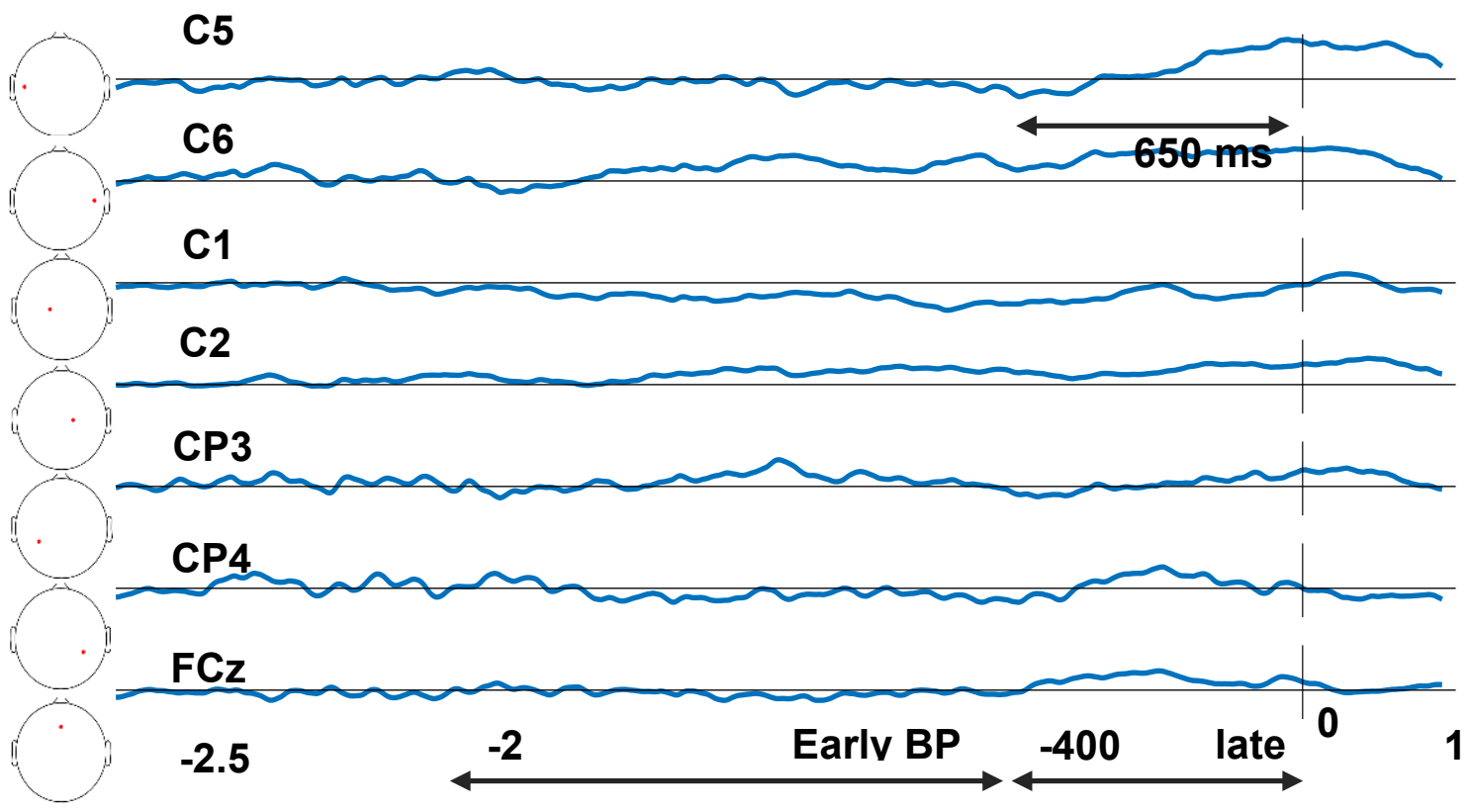

Figure 15. MRCP pilot results. Cortical activity is known to start prior to actual movement. As can be seen, the expected polarization 1-2 s before movement is shown, followed by the depolarization slightly before limb movement, lasting about $500 \mathrm{~ms}$.

under 30 minutes, this includes the EEG, EMG, GRF, and symptom tracking devices. Crucial for the exploration of active gait neural exploration in cognitively impaired groups, whose progression makes extended recordings and set-up times unfeasible.

Figure 15 shows the cortical activity, averaged over 155 trials. The clear polarizations before gait initiation show the sensitivity required to extract the biomarkers under the proposed paradigm. The early BP is associated with the activation at the pre-SMA, SMA, and Boardman area. The late BP is associated with activation of the PMC and is indicative of differences between PD and healthy controls during upper limb finger tapping studies. The signal-to-noise ratio was $8.54 \pm 4.33 \mathrm{~dB}$ indicating the dry electrode and 16 channel EEG DAQ provide comparable signal quality for MRCP exploration to that of a gold standard $\mathrm{Ag} / \mathrm{AgCl}$ wet electrode setup. 


\subsection{Discussion}

This study resulted in the development of a wearable suite capable of capturing neural patterns during active gait paradigms. Although there are plenty of commercially available devices that can deliver gold-standard-level individual sensors recordings[156], [176], our system allows for co-registration of all sensors within the suite from EEG at the top of the head to GRF at the plant of the feet, as well as objective symptom tracking. Our sensors' design focused on providing comparable signal quality to that of gold standards. In the case of EEG, our device was designed to meet the recommended impedance range during set up and throughout the recording. Additionally, the developed device showed comparable SNR [166], [170] and relevant bandwidth [159] responses to that of gold standard devices. This study also led to the development and validation of a novel device capable of quantifying the PD stage using an objective measure of bradykinesia. Whereas current practice still relies on a clinical measure that relies on the experience of an SMD [177], several groups have aimed at staging PD using objective measures. These measures range from a camera-based system that resulted in a PD stage classification of $88 \%$ [178], to an inertial measurement unit to record kinematics that resulted in a 90\% accuracy [179], and a microphone to record voice signatures that resulted in a UPDRS prediction accuracy of $96.2 \%$. In addition to a high UPDRS predicted accuracy of 95\%, our symptom tracking device also allows for real-time behavioral tracking with internet connectivity and the ability to co-register to electrophysiological measures from EEG and EMG and kinematic and kinetic measures from GRF and the inertial measurement units located on each device within the suite. Although sensors within the suite were tested and their potential use verified individually. Because PwP suffer from cognitive decline, it is not feasible to extend 
setup times or recordings due to physical and mental strain. Thus, further work should focus on employing the complete sensor suite on a cognitively impaired group of PwP to test longevity and comfort [173]. 


\section{CHAPTER 4 NEURAL BIOMARKERS OF FREEZING OF GAIT IN PARKINSON'S DISEASE PATIENTS}

\subsection{Introduction}

Persons with PD demonstrate gait impairments that usually increase with disease progression. It is well understood that PD is characterized by dopaminergic loss, leading to decreased executive function causing motor symptoms such as tremors, bradykinesia, dyskinesia, and FoG as well as non-motor symptoms such as loss of smell. However, PwP often lists FoG as one of the most debilitating symptoms. There have been five nonexclusive mechanism postulations that aim to explain the possible PD physiological alterations leading to FoG. This study will focus on exploring the second hypothesis, which states that FoG is caused by a problem with the central drive and automaticity of movement. It has been proposed that FoG may be due to the loss of automatic updating of motor programs by the dysfunctional basal ganglia in PD [54], [180]. Characterized by the inability to perform dual tasks[8], [68]. Previous studies propose that the excitatory drive from the STN, which projects to the cerebellar cortex, leads to inhibition of the deep cerebellar nuclei, resulting in the inability to perform previously known tasks[8], [69]. This problem with the automaticity of movement has also been suggested to be caused by the cross-talk of the basal ganglia inputs from the motor, cognitive, and limbic cortical areas, which regularly complement each other in unaffected persons [8]. This model suggests that there is synchronous activation from the basal ganglia, which inhibits gait control from brainstem regions. This model is further supported by Gilat et al. who reasons that with early PD, the frontal-parietal cortices are unaffected, allowing for gait to be controlled by goal-directed strategies. Whereas in later stages, as gait control becomes less automated, 
cross-talk between these competing regions leads to worsening gait executing while dual tasking[68].

To further understand this theory, clear biomarkers associated with gait and the physiological response from muscular activity in preparation to and during active gait are needed. These markers will help to better understand FoG which in turn may lead to targeted intervention for improved symptom treatment.

\subsection{Related Works}

In terms of neural activity, several previous studies have focused on PD to study the effects of self-paced upper limb activities while recording EEG activity[50], [99], [106][109], [111]. It has been shown that a negative potential, which appears 1-2 s before the limb movement, is sensed at the central EEG electrodes. This potential is referred to as the BP and is divided into early and late BP. Early BP occurs 1-2 s before limb movement, this is associated with activation at the pre-SMA, SMA, and Bordmann area 6 [109]. The late BP occurs between $400-500 \mathrm{~ms}$ before limb movement and is associated with activation of the primary motor cortex [110]. Studies have shown that early BP can be used as a marker for motor preparation and is reduced in PwP when compared to healthy controls [108], [111], [181]. Whereas late BP is overactivated and is theorized to be due to the compensation mechanisms to achieve limb control in the absence of information handover from the basal ganglia to the SMA [108]. These results have been largely reproduced only in PwP while performing upper limb tasks [109]. In terms of the lower limbs, previous studies have focused on the difference between PwP and healthy controls [113], or in finding relations between stride length changes and MRCP in PwP with and without FoG [114]. 
In addition to the MRCPs, time-frequency analysis is another method for evaluating neural patterns. A frequency-specific power increase or decrease in neural activity is known as an ERS and ERD, respectively [118]. In nominal function, beta synchronization following movement onset antagonizes movement and help re-establish a postural set [182]. Whereas desynchronization is related to the preparation of motor responses and motor selection [128]. In movement disorders and stroke patients, it has been shown that when patients are required to perform previously learned tasks, available resources for goal-driven tasks are lessened, leading to inaccurate production of movement, this is especially salient when multitasking (Frequency-specific biomarkers in neurodegenerative disorders: Implications of alpha and beta oscillations in motor behavior). In a PD-FoG study that aimed to find differences in EEG oscillations during a choice-reaction task, beta and alpha bands showed a reduction in preparatory ERD and delayed ERS, found after movement onset [183]. Tard et al. found a decrease in beta ERD during upper limb freezing in PD, this finding suggests that beta oscillations are involved in active immobilization and the pathogenesis of bradykinesia [128]. Attenuated alpha and low-beta ERD is a marker of impaired motor preparation in PD [184], which has been suggested to be caused by the reduction in $\mathrm{BG}$ involvement or due to the hypersynchronized thalamocortical activity [111], [129].

This study built on these previous studies for protocol design, data processing, and behavioral metric reporting, its main aims are to 1 . To explore noninvasive cortical biomarkers associated with the decoupling of BG-SMA that causes SM-PMC disruptions in PD patients with FoG. 2. To validate the use of the advanced signal processing algorithms and sensor fusion for ensuring related cortical potentials are rid of stationary 
and non-stationary artefacts during active gait. 3 . To investigate time-frequency and eventrelated alterations in PD patients with and without FoG during active gait.

\subsection{Materials and Methods}

Patients were recruited from the Movement Disorder Institute at the Sheba Medical Center. Each patient was approached by their Neurologist who explained the inclusion and exclusion criteria. Healthy controls were recruited by research collaborators and confirmed for inclusion in the study [115], [185].

\subsubsection{Participants and Experimental Design}

As this is an exploratory study; there is not enough data to establish a valid power analysis. Following similar studies delving into cortical potentials for PD [107], [109], [112], [114], [186], two experimental groups were investigated in this study; $10 \mathrm{PD}$ patients with FoG, and 5 age-matched HC (Healthy Controls), for a total of 15 subjects, whose demographics are shown in Table 3. Each PD participant was instructed to arrive on the day of the recording during the OFF dopaminergic state, after at least 12 hours of medication withdrawal. Inclusion criteria included PD participants over 50 years of age who had been diagnosed with idiopathic PD, who were able to walk $100 \mathrm{~m}$ OFF dopaminergic medication without assistance. Exclusion criteria included significant comorbidities, recent surgeries, or orthopedic impediments. HCs were included if over 50 years of age, able to walk without assistance, and had no neurological disease that could affect their gait. 
Table 3 .

Subject demographics (mean \pm standard deviation).

\begin{tabular}{|c|c|c|c|}
\hline & $P D$ & $H C$ & $p$-value ${ }^{a}$ \\
\hline Gender (male/female) & $12 / 3$ & $3 / 5$ & 0.042 \\
\hline Age & $67.7 \pm 8.0$ & $63 \pm 8.5$ & 0.099 \\
\hline Montreal Cognitive Assessment & $22.4 \pm 4.0$ & $25.8 \pm 2.3$ & 0.029 \\
\hline$U P D R S$ & $17.8 \pm 7.0$ & $\mathrm{~N} / \mathrm{A}$ & $\mathrm{N} / \mathrm{A}$ \\
\hline Disease Duration & $10.5 \pm 7.0$ & $\mathrm{~N} / \mathrm{A}$ & N/A \\
\hline \multicolumn{4}{|c|}{$\begin{array}{l}\text { PD: Parkinson's Disease; HC: Healthy Controls; UPDRS: Unified Parkinson's Disease Rating } \\
\text { ale. }\end{array}$} \\
\hline
\end{tabular}

\subsubsection{Data Acquisition}

Each participant performed three gait-related tasks: (1) standing still for one minute, (2) walking in a straight line, and (3) turning. Participants wore a portable EEG system (MicroMED®, Mogliano-Veneto, Italy), sampled at $2048 \mathrm{~Hz}$, which employed a 32 channel EEG montage consisting of Fz, Cz, Pz, Oz, Fp1, Fp2, F7, F8, F3, F4, FT9, FT10, FC5, FC6, T3, T4, C3, C4, CP5, CP6, TP9, TP10, T5, T6, P3, P4, O1, O2, PO9, and PO10 referenced at $\mathrm{C} 7$ and grounded on the left mastoid process. For gait cueing, surface EMG activity was recorded from four channels sampled at $2048 \mathrm{~Hz}$, located at the Tibialis Anterior and Gastrocnemius muscles of each leg. In addition to these objective measures, non-senor-based metrics in the form of clinical scales were also recorded. These include the United Parkinson's disease rating scale and the Montreal Cognitive Assessment. This study protocol was approved by the Institutional Review Board of the Sheba Medical 
Center and the data was recorded in the Center of Advanced Technologies in Rehabilitation of the medical center [185].

To compare the neural activities of each group, ERD/S were evaluated. ERD is the decrease in spectral power before movement 0.5-2 s before movement onset [102]. Because ERD is a measure of power change associated with movement intention, a baseline correction is required. This baseline is commonly recorded during a stationary period preceding the movement onset and subtracted from the epoch of activity of interest, which includes the period before and after movement onset. Because the features associated with ERD/S are frequency band-specific, the Morlet transform was used to detect the time-locked activity [187], [188].

\subsubsection{Signal Processing}

\subsubsection{Processing for ERD/S}

Raw EEG data was input into eeglab [174] for processing where a high-pass filter at 0.1 $\mathrm{Hz}$ was applied for. This was followed by CleanLine, which removes sinusoidal from ICA computed components or scalp channels using multi-tapering and a Thompson F-statistics. Then artefact subspace reconstruction was used to remove flatline channels, low-frequency drifts, noisy channels, short-time bursts, and incompletely repaired segments from the data. Following re-referencing to average, ICA was used to estimate cortical potential sources, then visual inspection was used to remove artefacts in frequency bands or source locations regarded as noise artefacts. To estimate the power spectrum a Morlet Wavelet was employed. This wavelet used 0.25 cycles at the lowest frequency which increased linearly to 1.56 at the highest frequency with a window of 17 samples $(66.41 \mathrm{~ms})$. After the power spectrum was computed. A baseline from two seconds before to one second before gait initiation was selected as a period of no activity. Each subjects' baseline was subtracted 
from their own trial and relevant frequency bins were separated for analysis. The relevant frequency bins were alpha at 8,10 , and $12 \mathrm{~Hz}$ and beta starting at $14 \mathrm{~Hz}$ and going up to $30 \mathrm{~Hz}$ by increments of $2 \mathrm{~Hz}$.

\subsubsection{Processing for MRCP}

Like when processing ERD/S, raw EEG data was input into eeglab then a bandpass filter was applied between 0.05 to $10 \mathrm{~Hz}$. This was followed by CleanLine, then artefact subspace reconstruction and re-referencing to average. Then ICA labels were applied and noisy components or trails were removed. A baseline from two seconds before to one second before gait initiation was selected as a period of no activity. Each subjects' baseline was subtracted from their trials.

\subsubsection{Statistical Analysis}

Following baseline removal and frequency binning for ERD/S, each subjects' trials were averaged to result in a single event for each subject, resulting in 5 samples for the HC. Because of the unbalanced sample sizes between the HC and the PDFoG group, 5 random subjects within the total PDFoG group were chosen for comparison against the HC group. An Independent t-test was employed to test the null hypothesis that sets of features from the PDFoG and HC, came from the same group. A p-value of less than 0.05 would indicate that the sets of features did not come from the same population, showing statistical proof of difference. 


\subsection{Results}
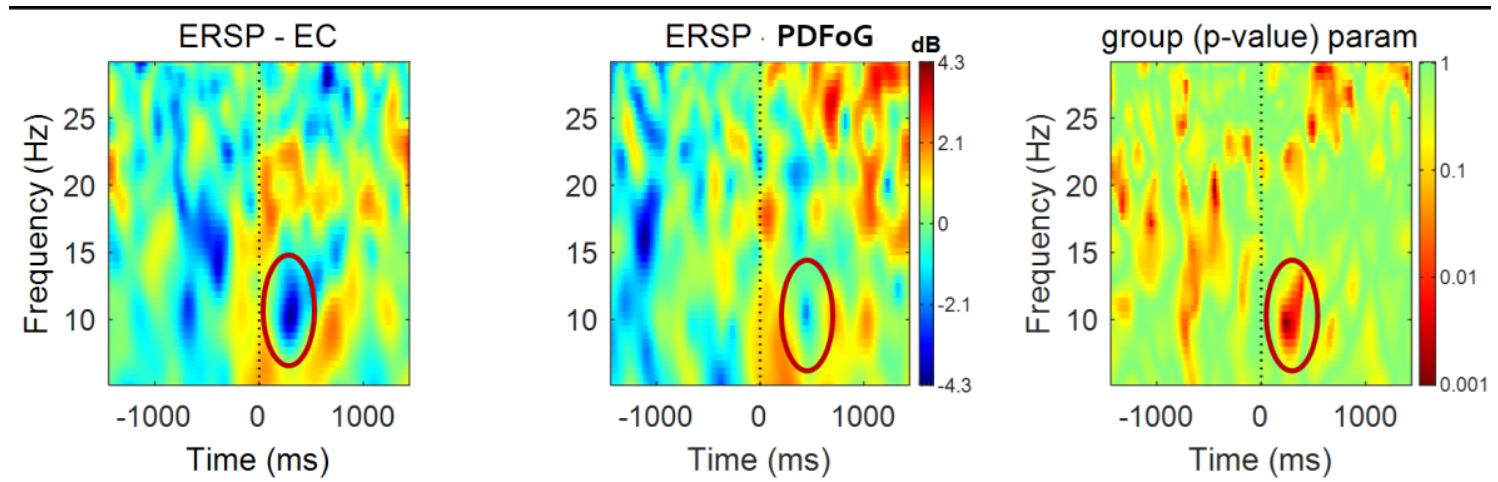

Figure 16. Time-frequency comparison. Each time-frequency decomposition plot is made up of the average activity for each group. The $\mathrm{x}$-axis represents the time $1500 \mathrm{~ms}$ before and $1500 \mathrm{~ms}$ after movement onset. The y-axis represents each frequency bin. Blue spots represent ERD and red spots represent ERS. The rightmost plot represents the intertrial coherence, with red representing periods of significance between group trials. The PDFoG group shows significantly less alpha and low beta desynchronization about $290 \mathrm{~ms}$ after the start of normal walking, compared with $\mathrm{HC}$, at the motor cortex. The statistical analysis supports this finding with the $\mathrm{p}<0.05$ for the comparison of frequency bins starting at $6 \mathrm{~Hz}$ and going to $12 \mathrm{~Hz}, 171$ ms after the start of normal walking.

As shown in Figure 16, ERD/S results showed a relative decrease of both alpha and low beta activity in the PDFoG group compared with the HC. This relative decrease was observed on $\mathrm{C} 3, \mathrm{CP} 6$, and $\mathrm{Cz}$, both before and after the start of normal walking. The following will present the channel-by-channel distribution of significant frequency bins that showed the difference between the subject groups. Channel C3, located near the motor cortex indicated significantly lower ERD in the alpha and low beta frequency bands after the start of normal walking, as illustrated in Figure 17. No other time of frequency bands showed significance when comparing the activity between the PDFoG and HC groups. 

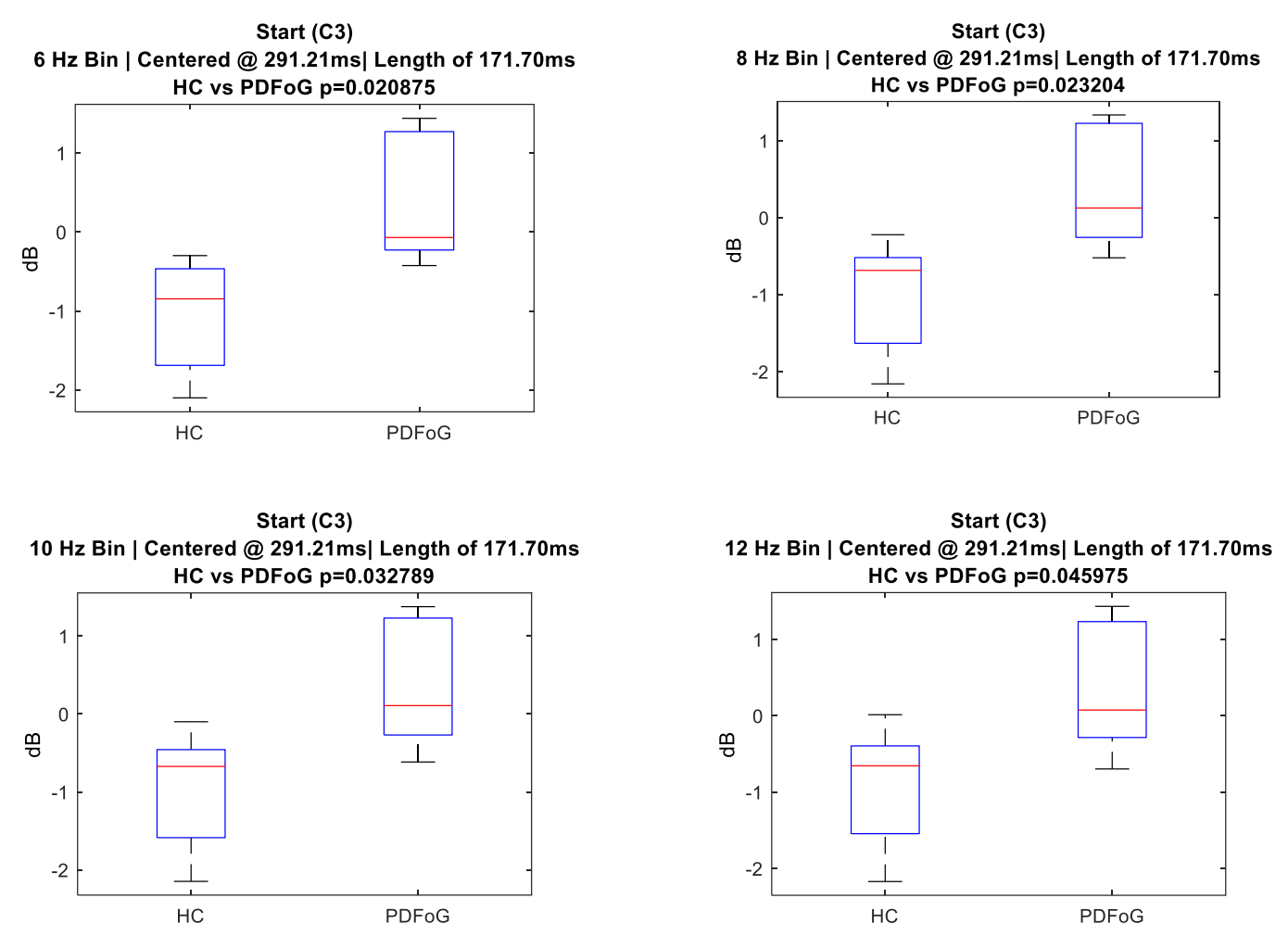

Figure 17. At C3, the PDFoG group shows significantly less alpha and low beta desynchronization about $290 \mathrm{~ms}$ after the start of normal walking, compared with HC.

The boxplot of the ERDs at CP6, $679 \mathrm{~ms}$ before the start of normal walking are presented in Figure 18. At the start of the normal walking event, channel CP6, located in the right hemisphere near the sensorimotor and parietal cortex also showed significant differences between the $\mathrm{HC}$ and PDFoG group with $\mathrm{p}<0.05$.

Start (CP6)

$6 \mathrm{~Hz}$ Bin | Centered @ -678.99ms| Length of $167.80 \mathrm{~ms}$ HC vs PDFoG $p=0.024677$

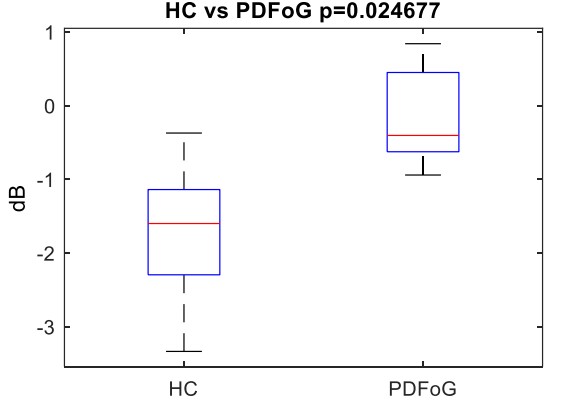

Start (CP6)

$6 \mathrm{~Hz}$ Bin | Centered @ -291.21ms| Length of $171.70 \mathrm{~ms}$ HC vs PDFoG $p=0.044859$

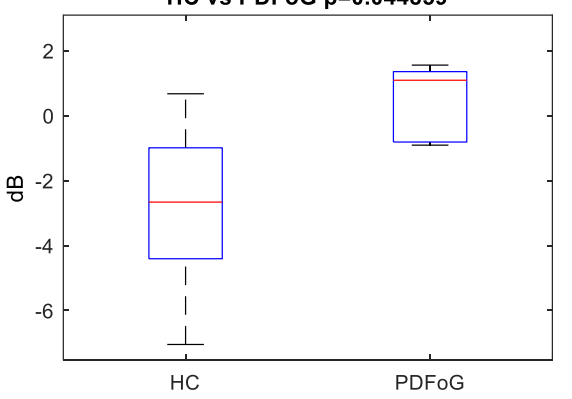



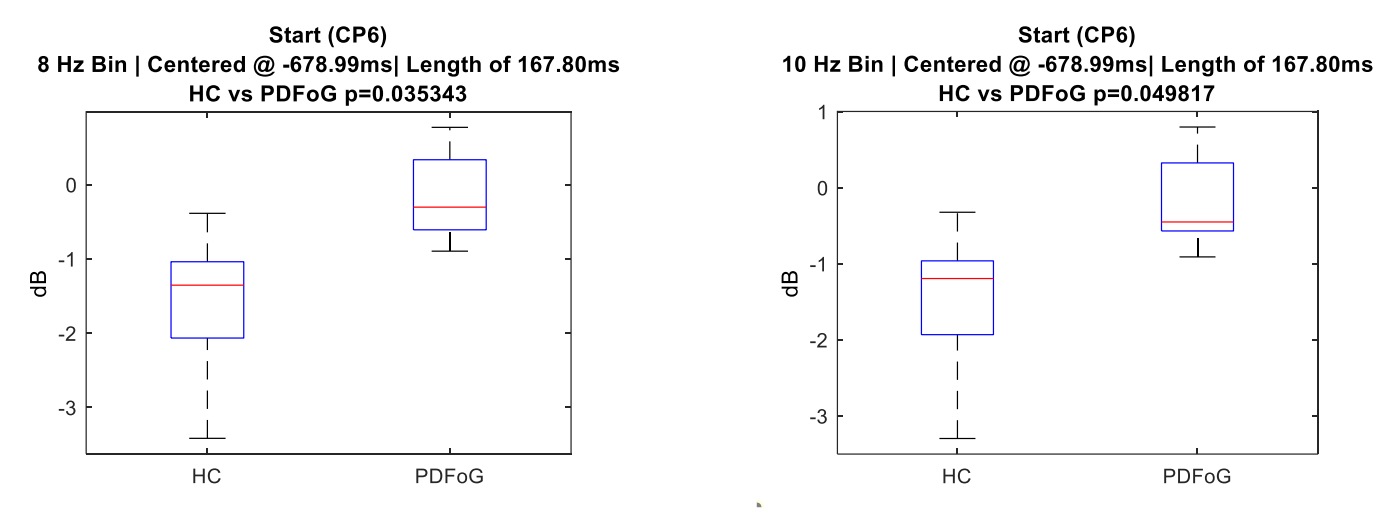

Figure 18. At CP6, the PDFoG group shows significantly less alpha desynchronization $680 \mathrm{~ms}$ and $290 \mathrm{~ms}$ before the start of normal walking, compared with $\mathrm{HC}$.

The Cz ERD $679 \mathrm{~ms}$ before and $290 \mathrm{~ms}$ after the start of normal walking are shown on the boxplots presented in Figure 19. Consistently with channels C3 and CP6, channel Cz, which is sensitive to the transition from normal walking to FoG events, also showed significant differences between the $\mathrm{HC}$ and PDFoG groups with $\mathrm{p}<0.05$.

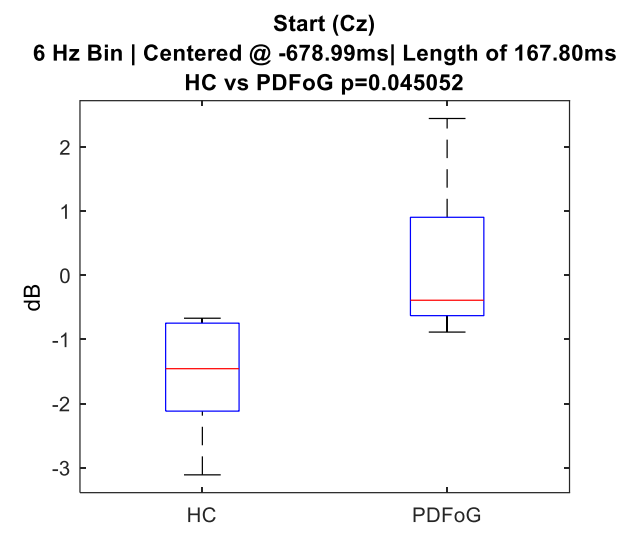

Start (Cz)

$6 \mathrm{~Hz}$ Bin | Centered @ 291.21ms| Length of $171.70 \mathrm{~ms}$ HC vs PDFoG $p=0.018205$
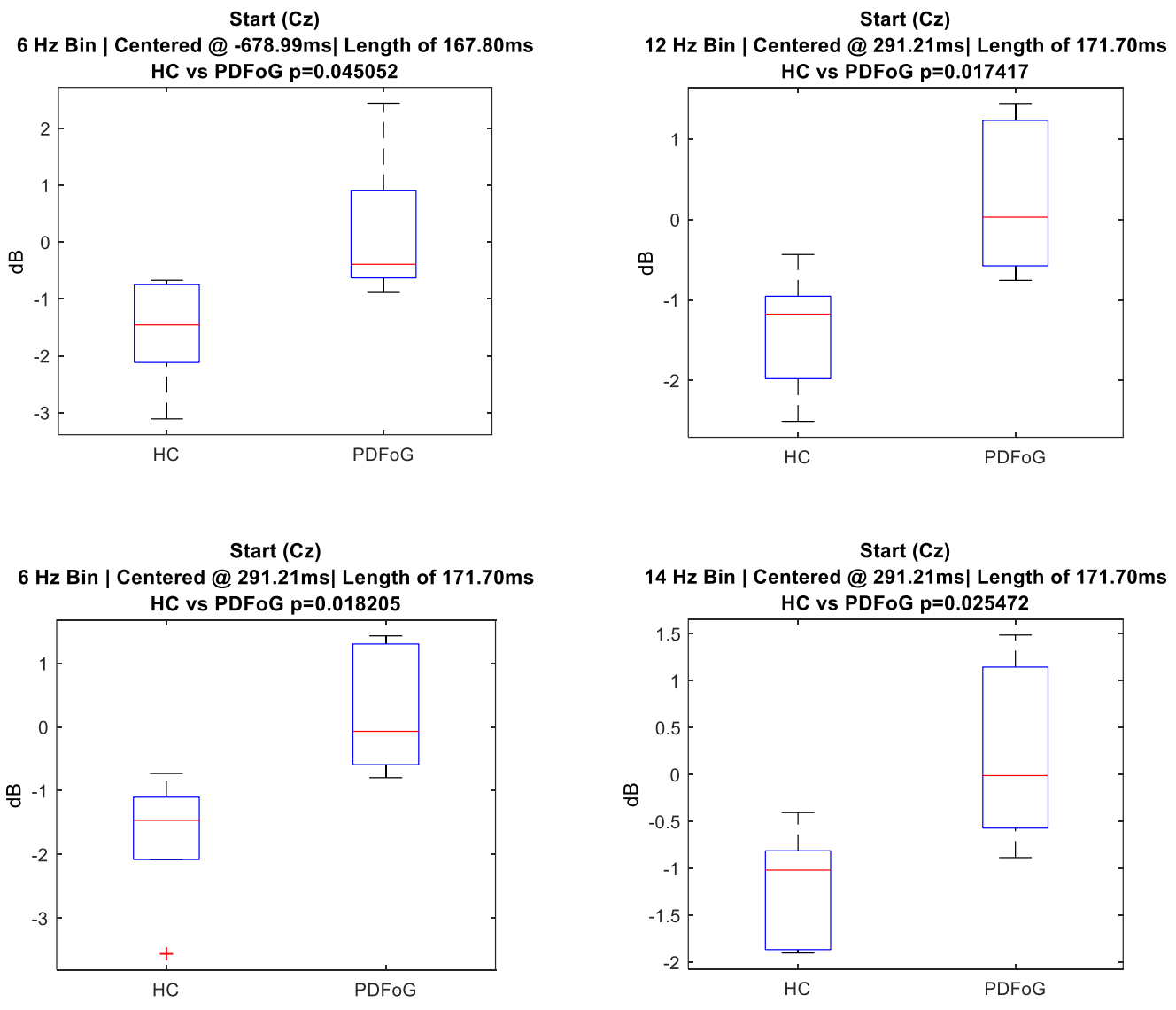

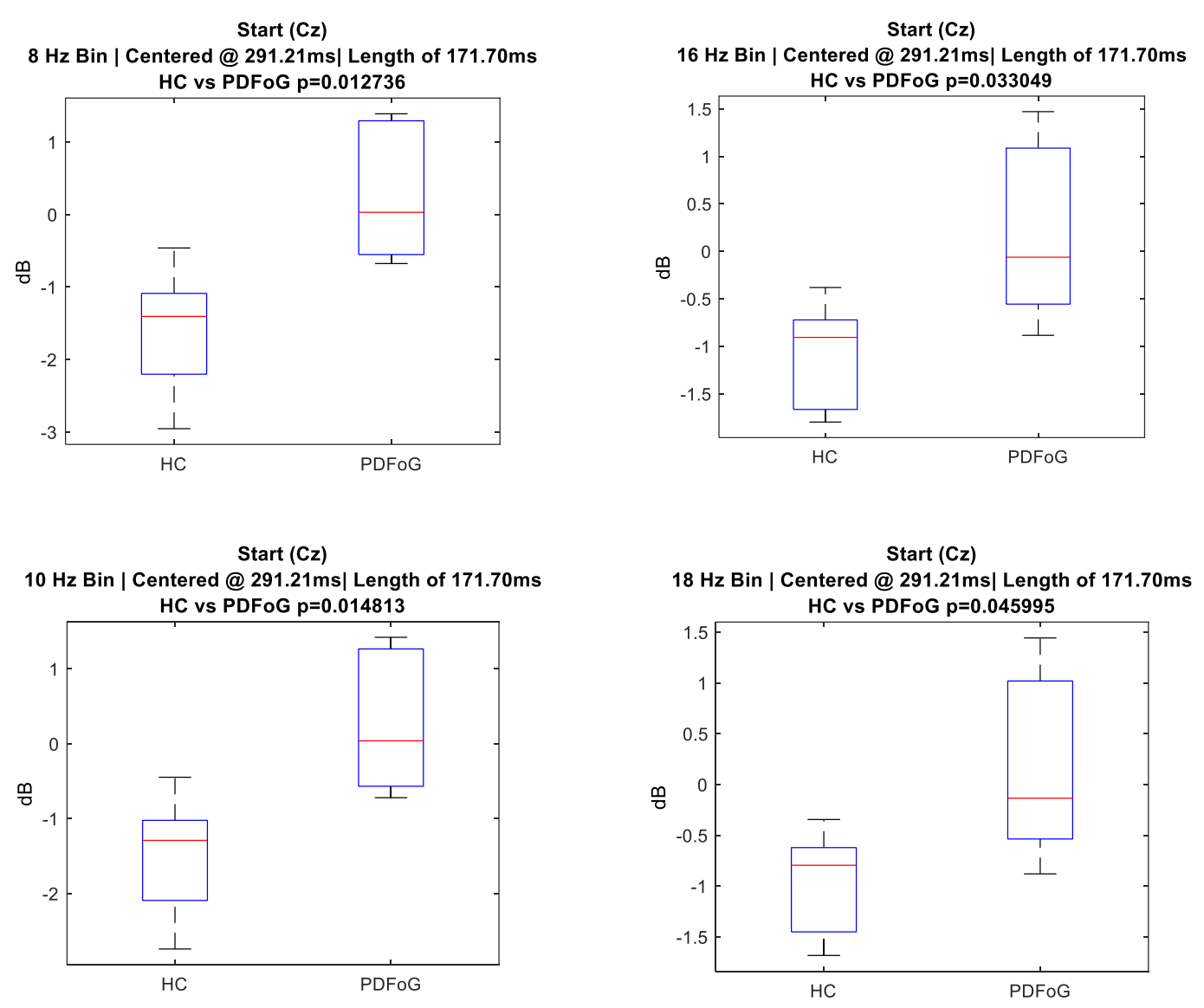

Figure 19. At $\mathrm{Cz}$, the PDFoG group shows significantly less alpha desynchronization $680 \mathrm{~ms}$ before and $290 \mathrm{~ms}$ after the start of normal walking, compared with $\mathrm{HC}$.

Following the observation that not all PDFoG exhibited FoG episodes during at least one gait task. The PDFoG group was split further into FoG+ and FoG-, the former included five patients who exhibited FoG events at least during one of the gait-related tasks and the latter included five patients who did not exhibit FoG events during any of the gait-related tasks but who had been previously diagnosed as having FoG symptoms. Applying this segmentation allowed for the exploration of differences between FoG + and FoG- groups in both the time-frequency and movement-related features. Conducting t-tests analysis between $\mathrm{HC}$, FoG-, and FoG+ resulted in significance when comparing the $26 \mathrm{~Hz}$ activity band on channel CP5 about $1 \mathrm{~s}$ after the end of normal walking with $\mathrm{p}<0.05$ within pairs of groups, Figure 20. 


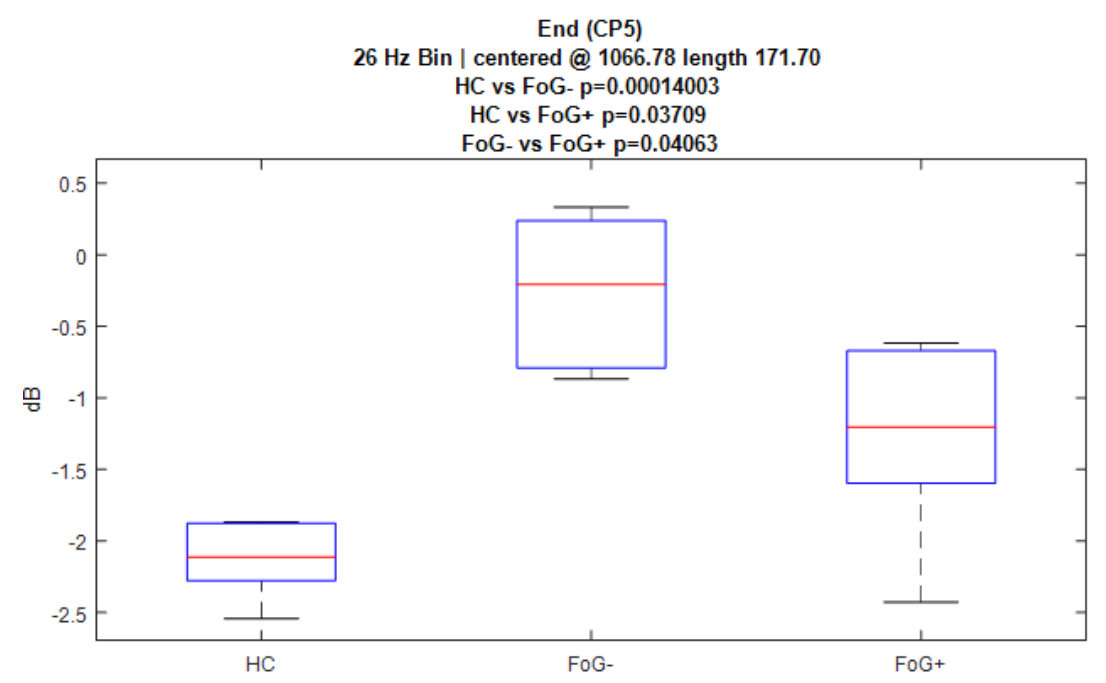

Figure 20. ERD differences between HC, FoG- and FoG + . Both FoG + and FoG- groups show significantly less beta desynchronization about $1 \mathrm{~s}$ after the stop of normal walking, compared with $\mathrm{HC}$. Additionally, at $26 \mathrm{~Hz}$ there is a significant difference between the FoG+ and FoGgroups.

In addition to the frequency domain evaluation (ERD/S) of the brain's preparation for movement execution, time-domain features (MRCP) were also explored. The post-hoc separation between PDFoG groups into FoG- and FoG+ was also explored for MRCP. A total of $31 \pm 2$ trials were averaged across groups. The averaging between $\mathrm{C} 3$ and $\mathrm{Cz}$, which constitute sensory and motor area respectively, showed an initial slope of motor potential (isMP) and the frontal peak of motor potential (fpMP) differences between the three groups. The isMP has been shown to originate over the primary motor cortex and represents activation. The fpMP originates from the supplementary motor area and has been shown to represent feedback up to $50 \mathrm{~ms}$ after movement onset. In PD, the isMP can be expected to be attenuated and the fpMP is delayed compared with HC [189]. The amplitudes of the MRCPs for each group are shown in Figure 21. The BP1, which occurs as far back as 2 seconds before movement onset could be ascertained due to the lack of baseline prior to the movement onset. 

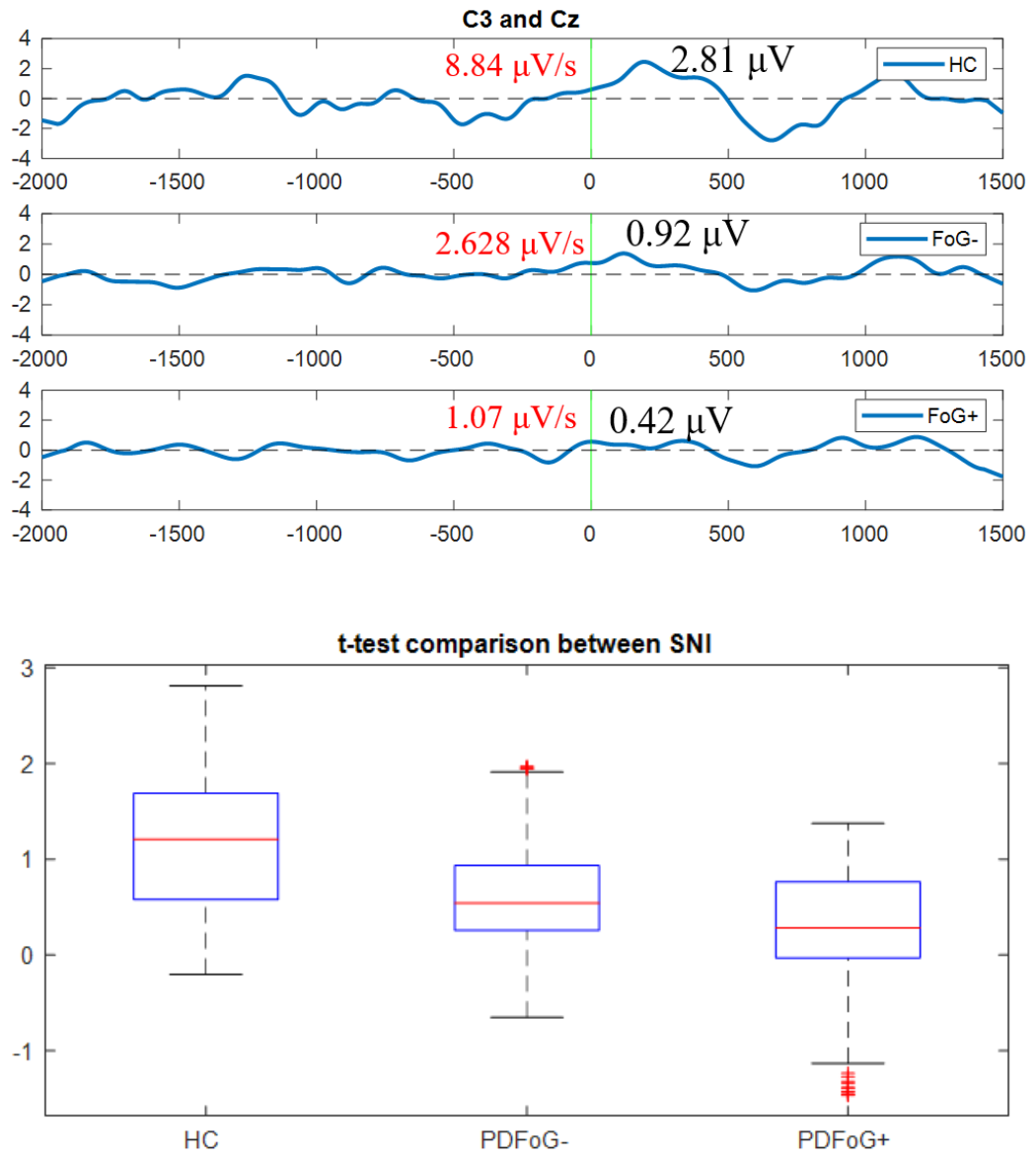

Figure 21. Movement-related cortical potentials. (Top) Averaged MRCP between 5 subjects for each group. Both $\mathrm{FoG}^{+}$and FoG- groups show significantly less isMP and fpMP in preparation and during the execution of normal walking, compared with HC. (Bottom) Additionally, fpMP is reduced in the FoG+ compared with the FoG- group.

For the Movement related cortical potential results. The analysis was limited to only analyzing the late BP. This limitation arose from the inability to ensure a period of inactivity $2 \mathrm{~s}$ before the movement onset. Instead, this analysis focused on the late BP or isMP, which indicates activation of the PMC. HC showed a clear polarization of late BP about $500 \mathrm{~ms}$ prior to the movement onset at a rate of $8.84 \mu \mathrm{V} / \mathrm{s}$, this is was attenuated in both FoG- at $2.63 \mu \mathrm{V} / \mathrm{s}$ and FoG+ at $1.07 \mu \mathrm{V} / \mathrm{s}$. After onset, fpMP and peak negativity were also attenuated in both FoG- at $0.92 \mu \mathrm{V} 132 \mathrm{~ms}$ after onset and FoG+ at $0.42 \mu \mathrm{V} 56 \mathrm{~ms}$ 
after onset compared with $\mathrm{HC}$ at $2.81 \mu \mathrm{V} 80 \mathrm{~ms}$ after onset, with the largest attenuation seen for FoG+.

\subsection{Discussion}

The purpose of this study was to test whether the proposed processing methods would be powerful enough to confirm the presence of neural patterns during active gait, which are affected in PDFoG during upper limb paradigms. Previous studies propose that the excitatory drive from the STN, which projects to the cerebellar cortex, leads to inhibition of the deep cerebellar nuclei, resulting in the inability to perform previously known tasks [8], [69]. The problem with the automaticity of movement has also been suggested to be caused by the crosstalk of the basal ganglia inputs from the motor, cognitive, and limbic cortical areas, which regularly complement each other in unaffected persons [8]. This model suggests that there is synchronous activation from the basal ganglia, which in term inhibits gait control from brainstem regions. Considering that the cortical activity over the SMA is reduced in PD before self-initiated movement because of the lack of innervation from the BG. In PD, the preparatory desynchronization (before movement initiation) is attenuated followed by reduced synchronization after movement execution, compared with HC. The results from this study support the lack of ERD in PDFoG before and after the start of normal walking. This lack of ERD may be caused by the affected movement programming [190], which is associated with reduced activity in the SMA [191]. Similarly, in PDFoG the ERS onset, before movement initiation, may be affected by earlier, elongated, increased in magnitude due to lack of ERD compared with HC.

As a feature of ERD/S explorations through EEG, the power change associated with a specific frequency band is measured relative to the power of a baseline period preceding 
the onset. In the literature, this period is commonly recommended to be recorded several seconds before the onset occurs. Additionally, because of the time required to develop and recover slow alpha rhythms, $10 \mathrm{~s}$ between task onsets is recommended [187]. Although this study had originally planned to include the periods of baseline and rest between trials, due to the pandemic, the originally proposed paradigms, which also aimed to explore the effects of dopaminergic medication on neural features, were not obtained. Instead, the dataset in this part of the study was obtained from our collaborators [115], [185]. With this dataset, the processing pipeline developed to attenuate motion artefacts from an active gait paradigm was tested. Although the short baseline may negatively affect the quality of the extracted neural patterns (by resulting in a suboptimal correction to the period of inactivity), the success of this trial shows the effectiveness of the proposed pipeline in confirming the presence of relevant neural patterns.

In terms of MRCP, previous studies have investigated movement-related cortical activity from noninvasive, scalp EEG recordings in PD patients[50], [99], [106]-[108], [111], mostly while undergoing self-paced upper limb motion. These studies have shown that the early BP can be used as a biomarker for motor preparation and is reduced in PD patients when compared to healthy controls when performing upper limb movements[108], [181], [192]. While the early BP is attenuated, these studies have shown that the late BP is overactivated and is considered as the compensation mechanism to achieve limb control in the absence of information handover from the basal ganglia to the SMA in PD patients[108]. To date, these results have been largely reproduced only in PD patients while performing upper limb tasks[109] due largely to potential motion artefacts associated with actual gait. In terms of lower limbs, previous studies have focused on the difference 
between PD patients and healthy controls[113], or in finding relations between stride length changes and MRCPs in PD patients with and without FoG[52]. In this active gait study, MRCP features show a decrease in the late BP slope of FoG+ compared to FoG- and HC. Such a reduction in slope has been matched with a reduction in stride length reduction [52], which has also been shown to indicated FoG events [193]. The reduced late BP amplitude may also indicate less neuronal signal projection from BG to the SMA, which may lead to reduced gait readiness, caudally leading to FoG or difficulty in self-initiated movement. Although others have reported higher late BP due to the compensatory mechanisms employed by PwP to execute motor control [108]. This study found a decreased late BP magnitude in the PD group, which was lowest for the FoG+ who exhibited FoG events at one or more of the gait tasks. This could be caused by the difference in the tasks administered, as this study explored changes during active gait, not index finger flexion. The additional cognitive load associated with gait execution, which in normal function is cued by the SLN network, becomes disrupted as a result of inhibition of the GPi/SNr pathway. This pathway breakdown causes engagement of the hyperdirect pathway between the SMA and STN resulting in affected cerebellar automatic gait processing.

\subsection{Conclusions}

The purpose of this study was to explore noninvasive cortical biomarkers associated with the decoupling of basal BG-SMA that causes the SMA-PMC disruptions. Considering that the cortical activity over the SMA is reduced before self-initiated movement because of lack of innervation from BG, this study hypothesized that the cortical activity over SMA associated with motor readiness would be different in PD patients with FoG events compared with HC. The major technical challenge in the proposed study was to obtain an 
accurate measurement of cognitive patterns associated with active gait, under the fact that the cortical recording may be largely distorted by gait-induced motion artefacts. This study found relative decreases of both alpha and beta activity in the PDFoG group compared with the $\mathrm{HC}$. These relative differences were observed on $\mathrm{C} 3, \mathrm{CP} 6$, and $\mathrm{Cz}$, in several time periods both before and after the start of normal walking. Following the observation that half of the PDFoG group did not exhibit FoG events at any point during the gait tasks, FoG- and FoG + subgroups were segmented from the PDFoG group. Statistical analysis showed significant differences in CP5 between FoG-, FoG+, and HC after the stop of normal walking. In terms of MRCP activity, the late BP segment showed a reduction in the FoG+ group compared to both the FoG- and HC groups.

Because of its large heterogeneity, PD is difficult to diagnose and manage. Furthermore, future works are needed in the exploration of neural biomarkers that differentiate PD subtypes during active gait paradigms. If better care strategies are to be developed, which take into consideration the case by case differences between PwP, a better understanding of how brain pathways are affected during gait with dopaminergic loss is required. This study provided support for the exploration of noninvasive biomarkers that may provide accurate assessments of pathophysiology in PD subgroups; this may lead to targeted intervention and improved symptom treatment for PD patients with FoG[54], [194]. Also, the technical innovations focused on dealing with the motion artefacts that have discouraged previous groups from performing active gait neural exploration. The results from this study support the feasibility of active gait MRCP research. Non-invasive biomarker research that relies on smart denoising algorithms for noise cancelation, and 
the implementation of ultra-wearable suites for the investigation of other diseases and in other fields. 


\section{REFERENCES}

[1] A. Reeve, E. Simcox, and D. Turnbull, "Ageing and Parkinson's disease: why is advancing age the biggest risk factor?," Ageing Res. Rev., vol. 14, no. 100, pp. 1930, Mar. 2014.

[2] T. Lebouvier et al., "The second brain and Parkinson's disease," Eur. J. Neurosci., vol. 30, no. 5, pp. 735-741, Sep. 2009.

[3] J. Lotharius and P. Brundin, "Pathogenesis of parkinson's disease: dopamine, vesicles and $\alpha$-synuclein," Nat. Rev. Neurosci., vol. 3, no. 12, pp. 932-942, Dec. 2002.

[4] A. Schrag, S. S. Zhelev, S. Hotham, R. D. Merritt, K. Khan, and L. Graham, "Heterogeneity in progression of prodromal features in Parkinson's disease," Parkinsonism Relat. Disord., vol. 64, pp. 275-279, Jul. 2019.

[5] T. A. Boonstra, H. van der Kooij, M. Munneke, and B. R. Bloem, "Gait disorders and balance disturbances in Parkinson's disease: clinical update and pathophysiology," Curr. Opin. Neurol., vol. 24, no. 4, pp. 461-471, Aug. 2008.

[6] B. R. Bloem, J. M. Hausdorff, J. E. Visser, and N. Giladi, "Falls and freezing of gait in Parkinson's disease: A review of two interconnected, episodic phenomena," Mov. Disord., vol. 19, no. 8, pp. 871-884, Aug. 2004.

[7] N. Giladi et al., "Freezing of gait in patients with advanced Parkinson's disease," $J$. Neural Transm., vol. 108, no. 1, pp. 53-61, Jan. 2001.

[8] J. G. Nutt, B. R. Bloem, N. Giladi, M. Hallett, F. B. Horak, and A. Nieuwboer, "Freezing of gait: moving forward on a mysterious clinical phenomenon," Lancet Neurol., vol. 10, no. 8, pp. 734-744, Aug. 2011.

[9] H. Stolze, S. Klebe, C. Zechlin, C. Baecker, L. Friege, and G. Deuschl, "Falls in frequent neurologicaldiseases," J. Neurol., vol. 251, no. 1, pp. 79-84, Jan. 2004.

[10] N. E. Allen, A. K. Schwarzel, and C. G. Canning, "Recurrent falls in Parkinson's disease: a systematic review.," Parkinsons. Dis., vol. 2013, p. 906274, 2013.

[11] J. Nonnekes, A. H. Snijders, J. G. Nutt, G. Deuschl, N. Giladi, and B. R. Bloem, "Freezing of gait: a practical approach to management," Lancet Neurol., vol. 14, no. 7, pp. 768-778, Jul. 2015.

[12] S. S. Rao, L. A. Hofmann, and A. Shakil, "Parkinson's disease: diagnosis and treatment.," Am. Fam. Physician, vol. 74, no. 12, pp. 2046-54, Dec. 2006.

[13] E. Tolosa, G. Wenning, and W. Poewe, "The diagnosis of Parkinson's disease," Lancet Neurol., vol. 5, no. 1, pp. 75-86, Jan. 2006. 
[14] R. Araújo, A. van Rumund, and B. Bloem, "To scan or not to scan your Parkinson patient: that is the question!," Pract. Neurol., p. practneurol-2019-002339, Sep. 2019.

[15] M. S. Okun, P. R. Zeilman, and N. P. F. (U.S.), Parkinson's Disease: Deep Brain Stimulation a Practical Guide for Patients and Families. National Parkinson Foundation, Incorporated, 2014.

[16] M. K. York, E. A. Wilde, R. Simpson, and J. Jankovic, "Relationship between neuropsychological outcome and DBS surgical trajectory and electrode location," $J$. Neurol. Sci., vol. 287, no. 1-2, pp. 159-171, Dec. 2009.

[17] J. N. Petry-Schmelzer et al., "Non-motor outcomes depend on location of neurostimulation in Parkinson's disease," Brain, Sep. 2019.

[18] Y. Okuma, "Practical approach to freezing of gait in Parkinson's disease," Practical Neurology, vol. 14, no. 4. BMJ Publishing Group, pp. 222-230, 2014.

[19] M. Plotnik and J. M. Hausdorff, "The role of gait rhythmicity and bilateral coordination of stepping in the pathophysiology of freezing of gait in Parkinson's disease," Mov. Disord., vol. 23, no. S2, pp. S444-S450, Jul. 2008.

[20] M. T. Barbe et al., "Gait and upper limb variability in Parkinson's disease patients with and without freezing of gait," J. Neurol., vol. 261, no. 2, pp. 330-342, Dec. 2014.

[21] J. M. Hausdorff, "Gait variability: methods, modeling and meaning.," J. Neuroeng. Rehabil., vol. 2, p. 19, Jul. 2005.

[22] J. Nantel, C. de Solages, and H. Bronte-Stewart, "Repetitive stepping in place identifies and measures freezing episodes in subjects with Parkinson's disease," Gait Posture, vol. 34, no. 3, pp. 329-333, Jul. 2011.

[23] J. Shah et al., "Increased foot strike variability in Parkinson's disease patients with freezing of gait," Park. Relat. Disord., vol. 53, pp. 58-63, Aug. 2018.

[24] A. Delval, L. Defebvre, and C. Tard, "Freezing during tapping tasks in patients with advanced Parkinson's disease and freezing of gait," PLoS One, vol. 12, no. 9, Sep. 2017.

[25] M. Scholten et al., "Neuromuscular correlates of subthalamic stimulation and upper limb freezing in Parkinson's disease," Clin. Neurophysiol., vol. 127, no. 1, pp. 610620, Jan. 2016.

[26] C. Moreau, C. Ozsancak, J.-L. Blatt, P. Derambure, A. Destee, and L. Defebvre, "Oral festination in Parkinson's disease: Biomechanical analysis and correlation with festination and freezing of gait," Mov. Disord., vol. 22, no. 10, pp. 1503-1506, Jul. 2007. 
[27] S. T. Nemanich and G. M. Earhart, "Freezing of gait is associated with increased saccade latency and variability in Parkinson's disease," Clin. Neurophysiol., vol. 127, no. 6, pp. 2394-2401, Jun. 2016.

[28] É. Parmentier, V. De Pasqua, K. D’Ostilio, F. Depierreux, G. Garraux, and A. Maertens de Noordhout, "Correlation between deep brain stimulation effects on freezing of gait and audio-spinal reflex," Clin. Neurophysiol., vol. 129, no. 10, pp. 2083-2088, Oct. 2018.

[29] E. Heremans, E. Nackaerts, S. Broeder, G. Vervoort, S. P. Swinnen, and A. Nieuwboer, "Handwriting Impairments in People with Parkinson's Disease and Freezing of Gait," Neurorehabil. Neural Repair, vol. 30, no. 10, pp. 911-919, Nov. 2016.

[30] M. J. Georgiades et al., "Investigating motor initiation and inhibition deficits in patients with Parkinson's disease and freezing of gait using a virtual reality paradigm," Neuroscience, vol. 337, pp. 153-162, Nov. 2016.

[31] H. K. Park et al., "Gait freezing and speech disturbance in Parkinson's disease," Neurol. Sci., vol. 35, no. 3, pp. 357-363, Mar. 2014.

[32] P. Arias, N. Espinosa, V. Robles-García, R. Cao, and J. Cudeiro, “Antagonist muscle co-activation during straight walking and its relation to kinematics: Insight from young, elderly and Parkinson's disease," Brain Res., vol. 1455, pp. 124-131, May 2012.

[33] A. Nieuwboer, R. Dom, W. De Weerdt, K. Desloovere, L. Janssens, and V. Stijn, "Electromyographic profiles of gait prior to onset of freezing episodes in patients with Parkinson's disease.," Brain, vol. 127, no. Pt 7, pp. 1650-60, Jul. 2004.

[34] L. Alibiglou, A. Videnovic, P. J. Planetta, D. E. Vaillancourt, and C. D. MacKinnon, "Subliminal gait initiation deficits in rapid eye movement sleep behavior disorder: A harbinger of freezing of gait?," Mov. Disord., vol. 31, no. 11, pp. 1711-1719, Nov. 2016.

[35] I. Maidan, - Hagar Bernad-Elazari, E. Gazit, N. Giladi, J. M. Hausdorff, and A. Mirelman, "Changes in oxygenated hemoglobin link freezing of gait to frontal activation in patients with Parkinson disease: an fNIRS study of transient motorcognitive failures," J Neurol, 2015.

[36] H. Ama and L. Sjg, "Abnormal patterns of theta frequency oscillations during the temporal evolution of freezing of gait in Parkinson's disease," 2013.

[37] J. B. Toledo et al., "High beta activity in the subthalamic nucleus and freezing of gait in Parkinson's disease," Neurobiol. Dis., vol. 64, pp. 60-65, Apr. 2014. 
[38] S. T. Moore et al., "Autonomous identification of freezing of gait in Parkinson's disease from lower-body segmental accelerometry," J. Neuroeng. Rehabil., vol. 10, no. 1, p. 19, Feb. 2013.

[39] J. S. Butler, C. Fearon, I. Killane, S. M. Waechter, R. B. Reilly, and T. Lynch, "Motor preparation rather than decision-making differentiates Parkinson's disease patients with and without freezing of gait," Clin. Neurophysiol., vol. 128, no. 3, pp. 463-471, Mar. 2017.

[40] C. C. Chen et al., "Subthalamic nucleus oscillations correlate with vulnerability to freezing of gait in patients with Parkinson's disease," Neurobiol. Dis., vol. 132, p. 104605, Dec. 2019.

[41] J. Syrkin-Nikolau et al., "Subthalamic neural entropy is a feature of freezing of gait in freely moving people with Parkinson's disease," Neurobiol. Dis., vol. 108, pp. 288-297, Dec. 2017.

[42] P. Fischer et al., "Alternating modulation of subthalamic nucleus beta oscillations during stepping," J. Neurosci., vol. 38, no. 22, pp. 5111-5121, May 2018.

[43] L. Storzer et al., "Bicycling and walking are associated with different cortical oscillatory dynamics," Front. Hum. Neurosci., vol. 10, no. FEB2016, 2016.

[44] V. Fraix et al., "Pedunculopontine nucleus area oscillations during stance, stepping and freezing in Parkinson's disease," PLoS One, vol. 8, no. 12, Dec. 2013.

[45] J. Crémers, K. D'Ostilio, J. Stamatakis, V. Delvaux, and G. Garraux, "Brain activation pattern related to gait disturbances in Parkinson's disease," Mov. Disord., vol. 27 , no. 12 , pp. $1498-1505$, Oct. 2012.

[46] A. L. Bartels and K. L. Leenders, "Brain imaging in patients with freezing of gait," Mov. Disord., vol. 23, no. S2, pp. S461-S467, Jul. 2008.

[47] K. Bharti et al., "Neuroimaging advances in Parkinson's disease with freezing of gait: A systematic review," NeuroImage: Clinical, vol. 24. Elsevier Inc., p. 102059, 01-Jan-2019.

[48] A. Fasano, T. Herman, A. Tessitore, A. P. Strafella, and N. I. Bohnen, "Neuroimaging of freezing of gait," J. Parkinsons. Dis., vol. 5, no. 2, pp. 241-254, Jun. 2015.

[49] N. Piramide et al., "Different Patterns of Brain Activity During Lower Limb Movements in Parkinson's Disease Patients With and Without Freezing of Gait (P5.8-011),"Neurology, vol. 92, no. 15 Supplement, 2019.

[50] P. Limousin et al., "The effects of posteroventral pallidotomy on the preparation and execution of voluntary hand and arm movements in Parkinson's disease," Brain, vol. 122, no. 2, pp. 315-327, Feb. 1999. 
[51] S. Stuart, R. Vitorio, R. Morris, D. N. Martini, P. C. Fino, and M. Mancini, "Cortical activity during walking and balance tasks in older adults and in people with Parkinson's disease: A structured review," Maturitas, vol. 113, pp. 53-72, Jul. 2018.

[52] M. Shoushtarian, A. Murphy, and R. Iansek, "Examination of central gait control mechanisms in Parkinson's disease using movement-related potentials," Mov. Disord., vol. 26, no. 13, pp. 2347-2353, Nov. 2011.

[53] A. M. Ardi Handojoseno, J. M. Shine, T. N. Nguyen, Y. Tran, S. J. G. Lewis, and H. T. Nguyen, "Analysis and prediction of the freezing of gait using EEG brain dynamics," IEEE Trans. Neural Syst. Rehabil. Eng., vol. 23, no. 5, pp. 887-896, Sep. 2015.

[54] J. M. Shine, S. L. Naismith, and S. J. G. Lewis, "The pathophysiological mechanisms underlying freezing of gait in Parkinson's Disease," J. Clin. Neurosci., vol. 18, no. 9, pp. 1154-1157, Sep. 2011.

[55] L. Roeder, T. W. Boonstra, and G. K. Kerr, "Corticomuscular control of walking in older people and people with Parkinson's disease," bioRxiv, p. 628016, May 2019.

[56] D. P. Murphy et al., "Electroencephalogram-based brain-computer interface and lower-limb prosthesis control: A case study," Front. Neurol., vol. 8, no. DEC, 2017.

[57] R. Atri et al., "Investigation of muscle activity during loaded human gait using signal processing of multi-channel surface EMG and IMU," in 2016 IEEE Signal Processing in Medicine and Biology Symposium, SPMB 2016 - Proceedings, 2017.

[58] M. R. Siddiquee, J. S. Marquez, R. Atri, R. Ramon, R. Perry Mayrand, and O. Bai, "Movement artefact removal from NIRS signal using multi-channel IMU data," Biomed. Eng. Online, vol. 17, no. 1, 2018.

[59] J. S. Márquez, R. Atri, M. R. Siddiquee, C. Leung, and O. Bai, "A Mobile, Smart Gait Assessment System for Asymmetry Detection Using Machine Learning-Based Classification," J. Biomed. Eng. Med. Devices, vol. 3, no. 2, p. 8, 2018.

[60] O. Bai, R. Atri, J. S. Marquez, and D.-Y. Fei, "Characterization of lower limb activity during gait using wearable, multi-channel surface EMG and IMU sensors," in 2017 International Electrical Engineering Congress, iEECON 2017, 2017.

[61] O. Bai et al., "A wireless, smart EEG system for volitional control of lower-limb prosthesis," in TENCON 2015 - 2015 IEEE Region 10 Conference, 2015, pp. 1-6.

[62] D. Bucher, "Central Pattern Generators," in Encyclopedia of Neuroscience, Elsevier Ltd, 2009, pp. 691-700.

[63] N. G. Pozzi et al., "Freezing of gait in Parkinson's disease reflects a sudden derangement of locomotor network dynamics," Brain, vol. 142, no. 7, pp. 20372050, 2019. 
[64] C. A. Thorn, H. Atallah, M. Howe, and A. M. Graybiel, "Differential Dynamics of Activity Changes in Dorsolateral and Dorsomedial Striatal Loops during Learning," Neuron, vol. 66, no. 5, pp. 781-795, Jun. 2010.

[65] A. Mishra, S. Singh, and S. Shukla, "Physiological and Functional Basis of Dopamine Receptors and Their Role in Neurogenesis: Possible Implication for Parkinson's disease," Journal of Experimental Neuroscience, vol. 12. SAGE Publications Ltd, 31-May-2018.

[66] V. Senft, T. C. Stewart, T. Bekolay, C. Eliasmith, and B. J. Kröger, "Inhibiting Basal Ganglia Regions Reduces Syllable Sequencing Errors in Parkinson's Disease: A Computer Simulation Study," Front. Comput. Neurosci., vol. 12, p. 41, Jun. 2018.

[67] R. Iansek and M. Danoudis, "Freezing of Gait in Parkinson's Disease: Its Pathophysiology and Pragmatic Approaches to Management," Mov. Disord. Clin. Pract., vol. 4, no. 3, pp. 290-297, May 2017.

[68] M. Gilat, A. Lígia Silva de Lima, B. R. Bloem, J. M. Shine, J. Nonnekes, and S. J. G. Lewis, "Freezing of gait: Promising avenues for future treatment," Parkinsonism and Related Disorders, vol. 52. Elsevier, pp. 7-16, 01-Jul-2018.

[69] S. J. G. Lewis and J. M. Shine, "The Next Step: A Common Neural Mechanism for Freezing of Gait,” Neuroscientist, vol. 22, no. 1. pp. 72-82, 2016.

[70] F. Prestori, L. Mapelli, and E. D'Angelo, "Diverse Neuron Properties and Complex Network Dynamics in the Cerebellar Cortical Inhibitory Circuit," Frontiers in Molecular Neuroscience, vol. 12. Frontiers Media S.A., 07-Nov-2019.

[71] R. Iansek, F. Huxham, and J. McGinley, "The sequence effect and gait festination in Parkinson disease: Contributors to freezing of gait?," Mov. Disord., vol. 21, no. 9, pp. 1419-1424, Sep. 2006.

[72] J. V Jacobs, F. B. Horak, V. K. Tran, and J. G. Nutt, "Multiple balance tests improve the assessment of postural stability in subjects with Parkinson's disease.," J. Neurol. Neurosurg. Psychiatry, vol. 77, no. 3, pp. 322-6, Mar. 2006.

[73] F. Magrinelli et al., "Pathophysiology of Motor Dysfunction in Parkinson's Disease as the Rationale for Drug Treatment and Rehabilitation," Parkinsons. Dis., vol. 2016, p. 9832839, 2016.

[74] J. V. Jacobs, J. G. Nutt, P. Carlson-Kuhta, M. Stephens, and F. B. Horak, "Knee Trembling During Freezing of Gait Represents Multiple Anticipatory Postural Adjustments," Exp. Neurol., vol. 215, no. 2, p. 334, Feb. 2009.

[75] M. Gilat et al., "Dysfunctional Limbic Circuitry Underlying Freezing of Gait in Parkinson's Disease," Neuroscience, vol. 374, pp. 119-132, Mar. 2018. 
[76] D. Cowie, P. Limousin, A. Peters, and B. L. Day, "Insights into the neural control of locomotion from walking through doorways in Parkinson's disease," Neuropsychologia, vol. 48, no. 9, pp. 2750-2757, Jul. 2010.

[77] D. Cowie, P. Limousin, A. Peters, M. Hariz, and B. L. Day, "Doorway-provoked freezing of gait in Parkinson's disease," Mov. Disord., vol. 27, no. 4, pp. 492-499, Apr. 2012.

[78] Y. Okuma, "Proceedings of MDPD 2013 Freezing of Gait and Falls in Parkinson's Disease," J Park. Dis, vol. 4, no. 2, pp. 255-260, 2014.

[79] Q. J. Almeida and C. A. Lebold, "Freezing of gait in Parkinson's disease: a perceptual cause for a motor impairment?," J. Neurol. Neurosurg. Psychiatry, vol. 81, no. 5, pp. 513-8, May 2010.

[80] R. G. Cohen, A. Chao, J. G. Nutt, and F. B. Horak, "Freezing of gait is associated with a mismatch between motor imagery and motor execution in narrow doorways, not with failure to judge doorway passability," Neuropsychologia, vol. 49, no. 14, pp. 3981-3988, Dec. 2011.

[81] E. Heremans, A. Nieuwboer, and S. Vercruysse, "Freezing of gait in Parkinson's disease: Where are we now? Topical collection on movement disorders," Curr. Neurol. Neurosci. Rep., vol. 13, no. 6, pp. 1-9, Apr. 2013.

[82] K. L. Possin, J. V. Filoteo, D. D. Song, and D. P. Salmon, "Spatial and Object Working Memory Deficits in Parkinson's Disease Are due to Impairment in Different Underlying Processes," Neuropsychology, vol. 22, no. 5, pp. 585-595, Sep. 2008.

[83] M. Amboni, A. Cozzolino, K. Longo, M. Picillo, and P. Barone, "Freezing of gait and executive functions in patients with Parkinson's disease," Mov. Disord., vol. 23, no. 3, pp. 395-400, Feb. 2008.

[84] D. Moher, A. Liberati, J. Tetzlaff, and D. G. Altman, "Preferred Reporting Items for Systematic Reviews and Meta-Analyses: The PRISMA Statement," PLoS Med., vol. 6, no. 7, p. e1000097, Jul. 2009.

[85] A. M. Janssen et al., "Cerebellar theta burst stimulation does not improve freezing of gait in patients with Parkinson's disease," J. Neurol., vol. 264, no. 5, pp. 963972, May 2017.

[86] I. Killane, G. Browett, and R. B. Reilly, "Measurement of attention during movement: Acquisition of ambulatory EEG and cognitive performance from healthy young adults," in Proceedings of the Annual International Conference of the IEEE Engineering in Medicine and Biology Society, EMBS, 2013, vol. 2013, pp. 63976400 . 
[87] M. Rudzińska et al., "Przyczyny i konsekwencje upadków w chorobie Parkinsona Badanie prospektywne," Neurol. Neurochir. Pol., vol. 47, no. 5, pp. 423-430, 2013.

[88] A. Maillet, P. Pollak, and B. Debû, "Imaging gait disorders in parkinsonism: A review," Journal of Neurology, Neurosurgery and Psychiatry, vol. 83, no. 10. BMJ Publishing Group, pp. 986-993, Oct-2012.

[89] M. Alam, H. E. Heissler, K. Schwabe, and J. K. Krauss, "Deep brain stimulation of the pedunculopontine tegmental nucleus modulates neuronal hyperactivity and enhanced beta oscillatory activity of the subthalamic nucleus in the rat 6hydroxydopamine model," Exp. Neurol., vol. 233, no. 1, pp. 233-242, Jan. 2012.

[90] P. Varriale et al., "Decreasing subthalamic deep brain stimulation frequency reverses cognitive interference during gait initiation in Parkinson's disease," Clin. Neurophysiol., vol. 129, no. 11, pp. 2482-2491, Nov. 2018.

[91] J. Arens, L. Storzer, J. Hirschmann, S. S. Dalal, A. Schnitzler, and M. Butz, "Freezing of gait does not modulate beta oscillations in mesial cortical motor areas," Movement Disorders, vol. 34, no. 3. John Wiley and Sons Inc., p. 436, 01-Mar-2019.

[92] M. Alam, H. E. Heissler, K. Schwabe, and J. K. Krauss, "Deep brain stimulation of the pedunculopontine tegmental nucleus modulates neuronal hyperactivity and enhanced beta oscillatory activity of the subthalamic nucleus in the rat 6hydroxydopamine model," Exp. Neurol., vol. 233, no. 1, pp. 233-242, Jan. 2012.

[93] H. L., T. L.M., and B. D.L., "Cortical phase amplitude coupling and pathologic synchronization during sleep in parkinson's disease," Ann. Neurol., vol. 84, no. Supplement 22, p. S194, 2018.

[94] G. Yogev, N. Giladi, C. Peretz, S. Springer, E. S. Simon, and J. M. Hausdorff, "Dual tasking, gait rhythmicity, and Parkinson's disease: Which aspects of gait are attention demanding?," Eur. J. Neurosci., vol. 22, no. 5, pp. 1248-1256, 2005.

[95] M. R. Siddiquee, S. M. S. Hasan, J. S. Marquez, R. N. Ramon, and O. Bai, "Accurate Vigilance Detection during Gait by Using Movement Artifact Removal," IEEE Access, vol. 8, pp. 51179-51188, 2020.

[96] A. H. Snijders, I. Toni, E. Ružička, and B. R. Bloem, "Bicycling breaks the ice for freezers of gait," Mov. Disord., vol. 26, no. 3, pp. 367-371, Feb. 2011.

[97] M. Gratkowski, L. Storzer, M. Butz, A. Schnitzler, D. Saupe, and S. S. Dalal, "Braincycles: Experimental setup for the combined measurement of cortical and subcortical activity in Parkinson's disease patients during cycling," Front. Hum. Neurosci., vol. 10, p. 685, Jan. 2017.

[98] R. Cunnington, R. Iansek, K. A. Johnson, and J. L. Bradshaw, "Movement-related potentials in Parkinson's disease. Motor imagery and movement preparation," Brain, vol. 120, no. 8, pp. 1339-1353, Aug. 1997. 
[99] A. P. Feve, N. Bathien, and P. Rondot, "Chronic Administration of L-Dopa Affects the Movement-Related Cortical Potentials of Patients With Parkinson's Disease," Clin. Neuropharmacol., vol. 15, no. 2, pp. 100-108, Apr. 1992.

[100] G. Pfurtscheller, K. Zalaudek, and C. Neuper, "Event-related beta synchronization after wrist, finger and thumb movement," Electroencephalogr. Clin. Neurophysiol. Mot. Control, vol. 109, no. 2, pp. 154-160, Apr. 1998.

[101] S. Stuart, R. Vitorio, R. Morris, D. N. Martini, P. C. Fino, and M. Mancini, "Cortical activity during walking and balance tasks in older adults and in people with Parkinson's disease: A structured review.," Maturitas, vol. 113, pp. 53-72, Jul. 2018.

[102] S. M. S. Hasan, M. R. Siddiquee, R. Atri, R. Ramon, J. Sebastian Marquez, and O. Bai, "Prediction of gait intention from pre-movement EEG signals: a feasibility study," J. Neuroeng. Rehabil., vol. 17, p. 50, 2020.

[103] J. Massion, "Movement, posture and equilibrium: Interaction and coordination," Progress in Neurobiology, vol. 38, no. 1. pp. 35-56, 1992.

[104] K. Takakusaki, "Functional Neuroanatomy for Posture and Gait Control," J. Mov. Disord., vol. 10, no. 1, pp. 1-17, 2017.

[105] K. Takakusaki, "Neurophysiology of gait: From the spinal cord to the frontal lobe," Movement Disorders, vol. 28, no. 11. pp. 1483-1491, 2013.

[106] M. Kuoppamäki, J. C. Rothwell, R. G. Brown, N. Quinn, K. P. Bhatia, and M. Jahanshahi, "Parkinsonism following bilateral lesions of the globus pallidus: performance on a variety of motor tasks shows similarities with Parkinson's disease.," J. Neurol. Neurosurg. Psychiatry, vol. 76, no. 4, pp. 482-90, Apr. 2005.

[107] J. P. . Dick et al., "The Bereitschaftspotential, 1-DOPA and parkinson's disease," Electroencephalogr. Clin. Neurophysiol., vol. 66, no. 3, pp. 263-274, Mar. 1987.

[108] J. P. R. DICK et al., "THE BEREITSCHAFTSPOTENTIAL IS ABNORMAL IN PARKINSON'S DISEASE," Brain, vol. 112, no. 1, pp. 233-244, Feb. 1989.

[109] D. Georgiev, F. Lange, C. Seer, B. Kopp, and M. Jahanshahi, "Movement-related potentials in Parkinson's disease," Clin. Neurophysiol., vol. 127, no. 6, pp. 25092519, Jun. 2016.

[110] C. H. M. Brunia, G. J. M. van Boxtel, and K. B. E. Böcker, Negative Slow Waves as Indices of Anticipation: The Bereitschaftspotential, the Contingent Negative Variation, and the Stimulus-Preceding Negativity. Oxford University Press, 2011.

[111] M. Jahanshahi, I. H. Jenkins, R. G. Brown, C. D. Marsden, R. E. Passingham, and D. J. Brooks, "Self-initiated versus externally triggered movements," Brain, vol. 118, no. 4, pp. 913-933, Aug. 1995. 
[112] R. Cunnington, R. Iansek, and J. L. Bradshaw, "Movement-related potentials in Parkinson's disease: External cues and attentional strategies," Mov. Disord., vol. 14, no. 1, pp. 63-68, Jan. 1999.

[113] M. Vidailhet, P. R. Atchison, F. Stocchi, P. D. Thompson, J. C. Rothwell, and C. D. Marsden, "The Bereitschaftspotential preceding stepping in patients with isolated gait ignition failure," Mov. Disord., vol. 10, no. 1, pp. 18-21, Jan. 1995.

[114] M. Shoushtarian, A. Murphy, and R. Iansek, "Examination of central gait control mechanisms in Parkinson's disease using movement-related potentials," Mov. Disord., vol. 26, no. 13, pp. 2347-2353, Nov. 2011.

[115] M. Günther et al., "Coupling between leg muscle activation and EEG during normal walking, intentional stops, and freezing of gait in Parkinson's disease," Front. Physiol., vol. 10, no. JUL, 2019.

[116] A. M. A. Handojoseno et al., "An EEG study of turning freeze in Parkinson's disease patients: The alteration of brain dynamic on the motor and visual cortex," in Proceedings of the Annual International Conference of the IEEE Engineering in Medicine and Biology Society, EMBS, 2015, vol. 2015-November, pp. 6618-6621.

[117] J. M. Shine et al., "Abnormal patterns of theta frequency oscillations during the temporal evolution of freezing of gait in parkinson's disease," Clin. Neurophysiol., vol. 125 , no. 3, pp. 569-576, Mar. 2014.

[118] G. Pfurtscheller, "Functional brain imaging based on ERD/ERS," in Vision Research, 2001, vol. 41, no. 10-11, pp. 1257-1260.

[119] A. Singh, R. Cole, A. Espinoza, D. Brown, J. Cavanagh, and N. Narayanan, "Frontal theta and beta oscillations during lower-limb movement in Parkinson's disease," bioRxiv, p. 634808, May 2019.

[120] C. C. Walton, J. M. Shine, L. Mowszowski, S. L. Naismith, and S. J. G. Lewis, "Freezing of gait in Parkinson's disease: Current treatments and the potential role for cognitive training," Restor. Neurol. Neurosci., vol. 32, no. 3, pp. 411-422, 2014.

[121] C. C. Walton et al., "Cognitive training for freezing of gait in Parkinson's disease: a randomized controlled trial," npj Park. Dis., vol. 4, no. 1, Dec. 2018.

[122] A. Singh, S. Kammermeier, A. Plate, J. Ilmberger, and K. Bötzel, "Basal ganglia oscillations during gait in Parkinsonian patients," Klin. Neurophysiol., vol. 42, no. 01, p. P237, Mar. 2011.

[123] A. Singh, A. Plate, S. Kammermeier, J. H. Mehrkens, J. Ilmberger, and K. Bötzel, "Freezing of gait-related oscillatory activity in the human subthalamic nucleus," Basal Ganglia, vol. 3, no. 1, pp. 25-32, Mar. 2013. 
[124] A. M. A. Handojoseno, J. M. Shine, T. N. Nguyen, Y. Tran, S. J. G. Lewis, and H. T. Nguyen, "Using EEG spatial correlation, cross frequency energy, and wavelet coefficients for the prediction of Freezing of Gait in Parkinson's Disease patients," in Proceedings of the Annual International Conference of the IEEE Engineering in Medicine and Biology Society, EMBS, 2013, vol. 2013, pp. 4263-4266.

[125] A. M. A. Handojoseno, J. M. Shine, T. N. Nguyen, Y. Tran, S. J. G. Lewis, and H. T. Nguyen, "The detection of Freezing of Gait in Parkinson's disease patients using EEG signals based on Wavelet decomposition," in Proceedings of the Annual International Conference of the IEEE Engineering in Medicine and Biology Society, $E M B S$, 2012, pp. 69-72.

[126] A. M. A. Handojoseno et al., "Prediction of freezing of gait using analysis of brain effective connectivity," in 2014 36th Annual International Conference of the IEEE Engineering in Medicine and Biology Society, EMBC 2014, 2014, vol. 2014, pp. 4119-4122.

[127] D. Williams et al., "Dopamine-dependent changes in the functional connectivity between basal ganglia and cerebral cortex in humans," Brain, vol. 125, no. 7, pp. $1558-1569,2002$.

[128] C. Tard et al., "Attention modulation during motor preparation in Parkinsonian freezers: A time-frequency EEG study," Clin. Neurophysiol., vol. 127, no. 12, pp. 3506-3515, Dec. 2016.

[129] P. Brown and C. D. Marsden, "What do the basal ganglia do?," Lancet (London, England), vol. 351, no. 9118, pp. 1801-4, Jun. 1998.

[130] P. Brown, "Oscillatory nature of human basal ganglia activity: Relationship to the pathophysiology of parkinson's disease," Movement Disorders, vol. 18, no. 4. pp. 357-363, 01-Apr-2003.

[131] J. S. Brittain and P. Brown, "Oscillations and the basal ganglia: Motor control and beyond," NeuroImage, vol. 85. Academic Press Inc., pp. 637-647, 15-Jan-2014.

[132] P. D. Velu et al., "Effect of visual feedback on the occipital-parietal-motor network in Parkinson's disease with freezing of gait," Front. Neurol., vol. 4 JAN, 2014.

[133] A. Jha et al., "Functional Connectivity of the Pedunculopontine Nucleus and Surrounding Region in Parkinson's Disease," Cereb. Cortex, vol. 27, pp. 54-67, 2017.

[134] C. Anidi et al., "Neuromodulation targets pathological not physiological beta bursts during gait in Parkinson's disease," Neurobiol. Dis., vol. 120, pp. 107-117, Dec. 2018.

[135] M. U. Ferraye et al., "Effects of pedunculopontine nucleus area stimulation on gait disorders in Parkinson's disease," Brain, vol. 133, no. 1, pp. 205-214, 2010. 
[136] B. W. Fling, R. G. Cohen, M. Mancini, J. G. Nutt, D. A. Fair, and F. B. Horak, "Asymmetric pedunculopontine network connectivity in parkinsonian patients with freezing of gait," Brain, vol. 136, no. 8, pp. 2405-2418, 2013.

[137] A. L. Ridgel and D. L. Ault, "High-cadence cycling promotes sustained improvement in bradykinesia, rigidity, and mobility in individuals with mildmoderate Parkinson's disease," Parkinsons. Dis., vol. 2019, 2019.

[138] L. Storzer et al., "Bicycling suppresses abnormal beta synchrony in the Parkinsonian basal ganglia," Ann. Neurol., vol. 82, no. 4, pp. 592-601, Oct. 2017.

[139] B. Bluett et al., "Neuroimaging and neuropsychological assessment of freezing of gait in Parkinson's disease," Alzheimer's Dement. Transl. Res. Clin. Interv., vol. 4, pp. 387-394, Jan. 2018.

[140] E. K. Miller and J. D. Cohen, "An Integrative Theory of Prefrontal Cortex Function,” Annu. Rev. Neurosci., vol. 24, no. 1, pp. 167-202, Mar. 2001.

[141] A. Singh, R. C. Cole, A. I. Espinoza, D. Brown, J. F. Cavanagh, and N. S. Narayanan, "Frontal theta and beta oscillations during lower-limb movement in Parkinson's disease," Clin. Neurophysiol., vol. 131, no. 3, pp. 694-702, Mar. 2020.

[142] J. M. Hausdorff, “Gait variability: methods, modeling and meaning.," J. Neuroeng. Rehabil., vol. 2, p. 19, Jul. 2005.

[143] S. Lord, K. Baker, A. Nieuwboer, D. Burn, and L. Rochester, "Gait variability in Parkinson's disease: An indicator of non-dopaminergic contributors to gait dysfunction?," J. Neurol., vol. 258, no. 4, pp. 566-572, Apr. 2011.

[144] V. Mishra et al., "Resting-state connectivity in Levodopa responsive and unresponsive Parkinson's disease patients with Freezing of Gait (4477)," Neurology, vol. 94, no. 15 Supplement, p. 4477, Apr. 2020.

[145] F. Silva et al., "Functional coupling of sensorimotor and associative areas during a catching ball task: A qEEG coherence study," Int. Arch. Med., vol. 5, no. 1, p. 9, 2012.

[146] Q. T. Ly et al., "Identifying montages that best detect the electroencephalogram power spectrum alteration during freezing of gait in Parkinson's disease patients," in Proceedings of the Annual International Conference of the IEEE Engineering in Medicine and Biology Society, EMBS, 2016, vol. 2016-October, pp. 6094-6097.

[147] Q. T. Ly et al., "Detection of turning freeze in Parkinson's disease based on Stransform decomposition of EEG signals," in Proceedings of the Annual International Conference of the IEEE Engineering in Medicine and Biology Society, EMBS, 2017, pp. 3044-3047. 
[148] A. M. A. Handojoseno et al., "Prediction of freezing of gait in patients with Parkinson's disease using EEG signals," in Studies in Health Technology and Informatics, 2018, vol. 246, pp. 124-131.

[149] V. R. Mishra et al., "Unique white matter structural connectivity in early-stage drugnaive Parkinson disease," Neurology, vol. 94, no. 8, pp. e774-e784, Feb. 2020.

[150] J. S. Marquez, C. Luca, M. R. Siddiquee, R. Mayrand, R. Atri, and O. Bai, "A wearable sensor device with internet connectivity for accurate movement assessment in Parkinson's patients," in 5th World Parkinson Congress 2019, 2019, p. 165.

[151] E. Niedermeyer and F. H. L. da Silva, "Electroencephalography: basic principles, clinical applications, and related ...," vol. 1. pp. 17-31, 2005.

[152] K. Kotowski, K. Stapor, J. Leski, and M. Kotas, "Validation of Emotiv EPOC+ for extracting ERP correlates of emotional face processing," Biocybern. Biomed. Eng., vol. 38, no. 4, pp. 773-781, Jan. 2018.

[153] A. J. Casson, "Wearable EEG and beyond," Biomedical Engineering Letters, vol. 9, no. 1. Springer Verlag, pp. 53-71, 08-Feb-2019.

[154] J. W. Y. Kam et al., "Systematic comparison between a wireless EEG system with dry electrodes and a wired EEG system with wet electrodes," Neuroimage, vol. 184, pp. 119-129, Jan. 2019.

[155] M. Teplan, “FUNDAMENTALS OF EEG MEASUREMENT,” 2002.

[156] W. D. Hairston et al., "Usability of four commercially-oriented EEG systems," $J$. Neural Eng., vol. 11, no. 4, Aug. 2014.

[157] R. Atri et al., "EMG-based energy expenditure optimization for active prosthetic leg tuning," in Proceedings of the Annual International Conference of the IEEE Engineering in Medicine and Biology Society, EMBS, 2017.

[158] R. Atri et al., "Smart data-driven optimization of powered prosthetic ankles using surface electromyography," Sensors (Switzerland), vol. 18, no. 8, 2018.

[159] U. Rashid, I. Niazi, N. Signal, and D. Taylor, "An EEG Experimental Study Evaluating the Performance of Texas Instruments ADS1299," Sensors, vol. 18, no. 11, p. 3721, Nov. 2018.

[160] H. Hinrichs, M. Scholz, A. K. Baum, J. W. Y. Kam, R. T. Knight, and H. J. Heinze, "Comparison between a wireless dry electrode EEG system with a conventional wired wet electrode EEG system for clinical applications," Sci. Rep., vol. 10, no. 1, pp. 1-14, Dec. 2020. 
[161] G. Di Flumeri, P. Aricò, G. Borghini, N. Sciaraffa, A. Di Florio, and F. Babiloni, "The Dry Revolution: Evaluation of Three Different EEG Dry Electrode Types in Terms of Signal Spectral Features, Mental States Classification and Usability," Sensors, vol. 19, no. 6, p. 1365, Mar. 2019.

[162] S. Verwulgen et al., "Determining comfortable pressure ranges for wearable eeg headsets," in Advances in Intelligent Systems and Computing, 2019, vol. 795, pp. $11-19$.

[163] P. Fiedler et al., "Contact Pressure and Flexibility of Multipin Dry EEG Electrodes," IEEE Trans. Neural Syst. Rehabil. Eng., vol. 26, no. 4, pp. 750-757, Apr. 2018.

[164] M. O’Sullivan, A. Temko, A. Bocchino, C. O’Mahony, G. Boylan, and E. Popovici, "Analysis of a low-cost eeg monitoring system and dry electrodes toward clinical use in the neonatal icu," Sensors (Switzerland), vol. 19, no. 11, Jun. 2019.

[165] X. Xing et al., "A High-Speed SSVEP-Based BCI Using Dry EEG Electrodes," Sci. Rep., vol. 8, no. 1, Dec. 2018.

[166] M. Jochumsen et al., "Quantification of Movement-Related EEG Correlates Associated with Motor Training: A Study on Movement-Related Cortical Potentials and Sensorimotor Rhythms," Front. Hum. Neurosci., vol. 11, p. 604, Dec. 2017.

[167] "Chloriding the Ag + wire."

[168] E.-R. Symeonidou, A. D. Nordin, W. D. Hairston, D. P. Ferris, and J. Crayton Pruitt, "Effects of Cable Sway, Electrode Surface Area, and Electrode Mass on Electroencephalography Signal Quality during Motion."

[169] J. J. Barron and C. Ashton, "The Effect of Temperature on Conductivity Measurement A REAGECON TECHNICAL PAPER.”

[170] K. Thielemans, T. Gili, E. F. Center, D. Mandic, and T. Radüntz, "Signal Quality Evaluation of Emerging EEG Devices," Front. Physiol.| www.frontiersin.org, vol. 9, p. 98, 2018.

[171] D. Zhu, J. Bieger, G. G. Molina, and R. M. Aarts, “A Survey of Stimulation Methods Used in SSVEP-Based BCIs," Comput. Intell. Neurosci., vol. 2010, p. 12, 2010.

[172] von Frau Filipa Alexandra Campos Viola, "Towards artifact-free auditory evoked potentials in cochlear implant users," p. 153, 2011.

[173] M. A. Lopez-Gordo, D. Sanchez-Morillo, and F. Pelayo Valle, "Dry EEG Electrodes," Sensors, vol. 14, pp. 12847-12870, 2014.

[174] A. Delorme and S. Makeig, "EEGLAB: an open source toolbox for analysis of single-trial EEG dynamics including independent component analysis," J. Neurosci. Methods, vol. 134, no. 1, pp. 9-21, Mar. 2004. 
[175] F. Karimi, J. Kofman, N. Mrachcz-Kersting, D. Farina, and N. Jiang, "Comparison of EEG spatial filters for movement related cortical potential detection," in Proceedings of the Annual International Conference of the IEEE Engineering in Medicine and Biology Society, EMBS, 2016, vol. 2016-October, pp. 1576-1579.

[176] M. Saito et al., "An in-shoe device to measure plantar pressure during daily human activity," Med. Eng. Phys., vol. 33, no. 5, pp. 638-645, Jun. 2011.

[177] "State of the Art Review," 2003.

[178] T. Khan, D. Nyholm, J. Westin, and M. Dougherty, "A computer vision framework for finger-tapping evaluation in Parkinson's disease," Artif. Intell. Med., vol. 60, no. 1, pp. 27-40, 2014.

[179] B. M. Eskofier et al., "Recent machine learning advancements in sensor-based mobility analysis: Deep learning for Parkinson's disease assessment," in Proceedings of the Annual International Conference of the IEEE Engineering in Medicine and Biology Society, EMBS, 2016, vol. 2016-October, pp. 655-658.

[180] R. Iansek, F. Huxham, and J. McGinley, "The sequence effect and gait festination in Parkinson disease: Contributors to freezing of gait?," Mov. Disord., vol. 21, no. 9, pp. 1419-1424, Sep. 2006.

[181] R. Cunnington, R. Iansek, J. L. Bradshaw, and J. G. Phillips, "Movement-related potentials in Parkinson's disease," Brain, vol. 118, no. 4, pp. 935-950, Aug. 1995.

[182] P. Brown and D. Williams, "Basal ganglia local field potential activity: Character and functional significance in the human," Clinical Neurophysiology, vol. 116, no. 11. Clin Neurophysiol, pp. 2510-2519, Nov-2005.

[183] P. Praamstra and P. Pope, "Slow brain potential and oscillatory EEG manifestations of impaired temporal preparation in Parkinson's disease," J. Neurophysiol., vol. 98, no. 5, pp. 2848-2857, Nov. 2007.

[184] E. Labyt et al., "Motor preparation is more impaired in Parkinson's disease when sensorimotor integration is involved," Clin. Neurophysiol., vol. 114, no. 12, pp. 2423-2433, Dec. 2003.

[185] Y. Miron-Shahar et al., "Excessive phase synchronization in cortical activation during locomotion in persons with Parkinson's disease," Park. Relat. Disord., vol. 65, pp. 210-216, Aug. 2019.

[186] E. L. Stegemöller, D. P. Allen, T. Simuni, and C. D. MacKinnon, "Motor cortical oscillations are abnormally suppressed during repetitive movement in patients with Parkinson's disease," Clin. Neurophysiol., vol. 127, no. 1, pp. 664-674, Jan. 2016. 
[187] G. Pfurtscheller and F. H. Lopes Da Silva, "Event-related EEG/MEG synchronization and desynchronization: Basic principles," Clinical Neurophysiology, vol. 110, no. 11. Elsevier, pp. 1842-1857, 01-Nov-1999.

[188] S. M. Shafiul Hasan, M. R. Siddiquee, and O. Bai, "Asynchronous Prediction of Human Gait Intention in a Pseudo Online Paradigm Using Wavelet Transform," IEEE Trans. Neural Syst. Rehabil. Eng., vol. 28, no. 7, pp. 1623-1635, Jul. 2020.

[189] M. Hallett, "Movement-related cortical potentials.," Electromyogr. Clin. Neurophysiol., vol. 34, no. 1, pp. 5-13, 1994.

[190] H. M. Wu et al., "Attenuated NoGo-related beta desynchronisation and synchronisation in Parkinson's disease revealed by magnetoencephalographic recording," Sci. Rep., vol. 9, no. 1, pp. 1-12, Dec. 2019.

[191] A. Berardelli, J. C. Rothwell, P. D. Thompson, and M. Hallett, "Pathophysiology of bradykinesia in parkinson's disease," Brain, vol. 124, no. 11. Oxford University Press, pp. 2131-2146, 2001.

[192] M. Jahanshahi and M. Hallett, "The Bereitschaftspotential: What Does It Measure and where Does It Come from?," in The Bereitschaftspotential, Boston, MA: Springer US, 2003, pp. 1-17.

[193] R. Chee, A. Murphy, M. Danoudis, N. Georgiou-Karistianis, and R. Iansek, "Gait freezing in Parkinson's disease and the stride length sequence effect interaction," Brain, vol. 132, no. 8, pp. 2151-2160, Aug. 2009.

[194] S. Stuart, R. Vitorio, R. Morris, D. N. Martini, P. C. Fino, and M. Mancini, "Cortical activity during walking and balance tasks in older adults and in people with Parkinson's disease: A structured review," Maturitas, vol. 113. Elsevier Ireland Ltd, pp. 53-72, 01-Jul-2018. 
VITA

JUAN SEBASTIAN MARQUEZ JARAMILLO

2017-2020 Ph.D. Electrical and Computer Engineering

Florida International University

Miami, Florida, USA

2017-2018 M.Sc. Electrical Engineering

Florida International University

Miami, Florida, USA

2013-2017 B.Sc. Biomedical Engineering, Biomechanics \& Biomaterials

Florida International University

Miami, Florida, USA

\section{PUBLICATIONS}

1. J. Sebastian Marquez, et al., Neural correlates of Freezing of Gait in Parkinson's disease: An Electrophysiology Mini-Review. Front. Neurol. (Accepted).

2. Shafiul Hasan SM, Siddiquee MR, Atri R, Ramon R, Marquez JS, Bai O. Prediction of gait intention from pre-movement EEG signals: a feasibility study. $J$ Neuroeng Rehabil. 2020;17(1):50. Published 2020 Apr 16. doi:10.1186/s12984-020-00675-5

3. M. R. Siddiquee, S. M. S. Hasan, J. S. Marquez, R. N. Ramon and O. Bai, "Accurate Vigilance Detection During Gait by Using Movement Artifact Removal," in IEEE Access, vol. 8, pp. 51179-51188, 2020, doi: 10.1109/ACCESS.2020.2980546.

4. CPS pressure-based sensing system for symmetry measurement, Patent number: $10,555,689$

5. M. R. Siddiquee, S.M.S. Hasan, J.S. Marquez, "Sensor Fusion in Human Cyber Sensor System for Motion Artifact Removal from NIRS Signal," 2019 12th International Conference on Human System Interaction (HSI), Richmond, VA, USA, 2019, pp. 192-196, doi: 10.1109/HSI47298.2019.8942617.

6. Masudur R Siddiquee, J Sebastian Marquez, et al. "Movement artefact removal from NIRS signal using multi-channel IMU data." BioMedical Engineering OnLine, Dec. 2018. 17:120.

7. Marquez, J. Sebastian, et al. "A Mobile, Smart Gait Assessment System for Asymmetry Detection Using Machine Learning-Based Classification." Omicsonline, 4 Oct. 2018, Journal of Biomedical Engineering and Medical Devices. 
8. Atri, R.; Marquez, J.S.; Leung, C.; Siddiquee, M.R.; Murphy, D.P.; Gorgey, A.S.; Lovegreen, W.T.; Fei, D.-Y.; Bai, O. Smart Data-Driven Optimization of Powered Prosthetic Ankles Using Surface Electromyography. Sensors 2018, 18, 2705.

9. Siddiquee, Masudur R., Marquez, J.S., et al. "Movement Artefact Removal from NIRS Signal Using Multi-Channel IMU Data." BioMedical Engineering OnLine 17 (2018): 120. PMC. Web. 18 Oct. 2018.

10. Bai, O., Atri, R., Marquez, J.S. and Fei, D.Y., 2017, March. Characterization of lower limb activity during gait using wearable, multi-channel surface EMG and IMU sensors. In Electrical Engineering Congress (iEECON), 2017 International (pp. 1-4). IEEE.

11. Atri, R., Marquez, J.S., Murphy, D., Gorgey, A., Fei, D., Fox, J., Lovegreen, W. and Bai, O., 2017, July. EMG-based energy expenditure optimization for active prosthetic leg tuning. In Engineering in Medicine and Biology Society (EMBC), 2017 39th Annual International Conference of the IEEE (pp. 394-397). IEEE.

12. Murphy, D.P., Bai, O., Gorgey, A.S., Fox, J., Lovegreen, W.T., Burkhardt, B.W., Atri, R., Marquez, J.S., Li, Q. and Fei, D.Y., 2017. electroencephalogram-Based Brain-computer interface and Lower-Limb Prosthesis control: A case study. Frontiers in neurology, 8, p.696.

13. Atri, R., Marquez, J.S., Murphy, D., Gorgey, A., Fei, D., Fox, J., Burkhardt, B., Lovegreen, W. and Bai, O., 2016, December. Investigation of muscle activity during loaded human gait using signal processing of multi-channel surface EMG and IMU. In Signal Processing in Medicine and Biology Symposium (SPMB), 2016 IEEE (pp. 1-6). IEEE. 